Florida International University FIU Digital Commons

\title{
Modeling of Crosstalk in High Speed Planar Structure Parallel Data Buses and Suppression by Uniformly Spaced Short Circuits
}

Gabriel A. Solana

Florida International University, gsolana816@gmail.com

DOI: $10.25148 /$ etd.FI12050215

Follow this and additional works at: https://digitalcommons.fiu.edu/etd

\section{Recommended Citation}

Solana, Gabriel A., "Modeling of Crosstalk in High Speed Planar Structure Parallel Data Buses and Suppression by Uniformly Spaced Short Circuits" (2012). FIU Electronic Theses and Dissertations. 606.

https://digitalcommons.fiu.edu/etd/606 


\title{
FLORIDA INTERNATIONAL UNIVERSITY
}

Miami, Florida

MODELING OF CROSSTALK IN HIGH SPEED PLANAR STRUCTURE PARALLEL DATA BUSES AND SUPPRESSION BY UNIFORMLY SPACED SHORT CIRCUITS

\author{
A thesis submitted in partial fulfillment of the \\ requirements for the degree of \\ MASTER OF SCIENCE \\ in \\ ELECTRICAL ENGINEERING \\ by \\ Gabriel Alejandro Solana
}




\section{To: Dean Amir Mirmiran}

\section{College of Engineering and Computing}

This thesis, written by Gabriel Alejandro Solana, and entitled, Modeling of Crosstalk in High Speed Planar Structure Parallel Data Buses and Suppression by Uniformly Spaced Short Circuits, having been approved in respect to style and intellectual content, is referred to you for judgment.

We have read this thesis and recommend that it be approved.

Stavros V. Georgakopoulos

Jean H. Andrian

Grover Larkins, Major Professor

Date of Defense: March 29, 2012

The thesis of Gabriel Alejandro Solana is approved.

Dean Amir Mirmiran

College of Engineering and Computing

Dean Lakshmi N. Reddi University Graduate School

Florida International University, 2012 


\section{DEDICATION}

To those who helped make this happen. 


\section{ACKNOWLEDGMENTS}

I wish to express my gratitude toward my major professor, Dr. Grover Larkins, for his multifaceted support in helping me assemble this master's thesis. His encouragement played a key role in my ability to grow from the many challenges encountered in this experience.

Many thanks to my wife, Marilen, for always believing in me and supporting me throughout this challenging stage of my life. Your patience toward the amount of time demanded of me to complete this work has been a silent sacrifice on your part that spoke volumes to me, I cannot appreciate that enough. Thank you.

I would like to thank my mother for her support and helping me realize my own potential.

Dr. Yuriy Vlasov, Kiar Holland and David Brenner, thank you all for allowing me all the opportunities to share my work with you and gain a better understanding for it. Many thanks to you gentlemen for your input on my techniques, approaches, and writing style.

To Oscar Silveira for opening his resources to me and helping me out at odd hours.

To all of the faculty members of the electrical and computer engineering department who had a hand in helping me reach this point in my career. No matter how small a detail or whether you realized you helped me or not, thank you.

To my family, thank you for your day to day support in both a mental and moral sense. You will never know how much I appreciate your being there for me. 


\title{
ABSTRACT OF THE THESIS
}

MODELING OF CROSSTALK IN HIGH SPEED PLANAR STRUCTURE PARALLEL DATA BUSES AND SUPPRESSION BY UNIFORMLY SPACED SHORT CIRCUITS

by

\author{
Gabriel Alejandro Solana
}

Florida International University, 2012

Miami, Florida

Professor Grover Larkins, Major Professor

The aim of this thesis is to identify coupling mechanisms for three line microstrip, stripline and microstrip with dielectric overlay structures as either inductive or capacitive, quantify through simulation and measurement the amount of crosstalk to be expected in terms of scattering parameters. A new method of crosstalk suppression is implemented into each three line structure by placing uniformly spaced short circuits down the length of the center transmission line.

All structures were simulated over various physical and electrical parameters. Select microstrip structures, shielded and unshielded, were fabricated and measured to validate the effectiveness of the shielding technique. Shielding effectiveness was calculated from the measurements, and their results showed that the isolation between lines was increased by up to $20 \mathrm{~dB}$. 


\section{TABLE OF CONTENTS}

CHAPTER

PAGE

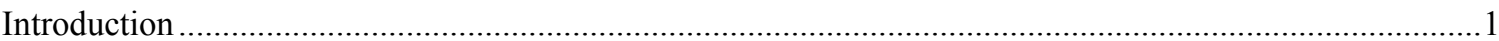

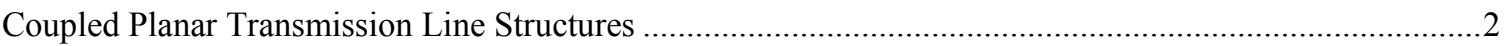

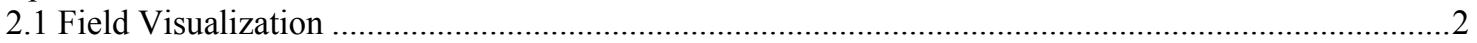

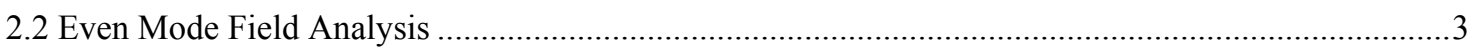

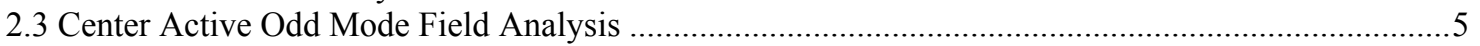

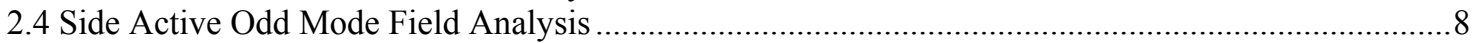

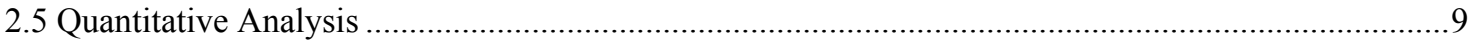

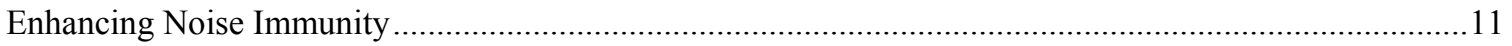

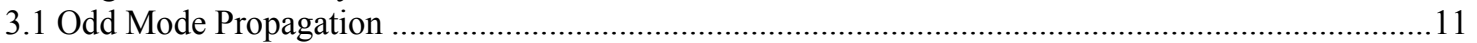

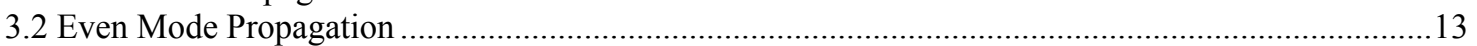

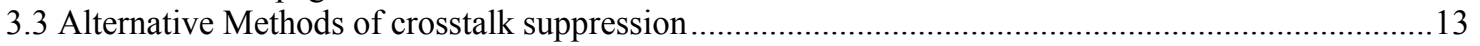

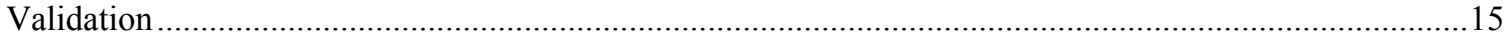

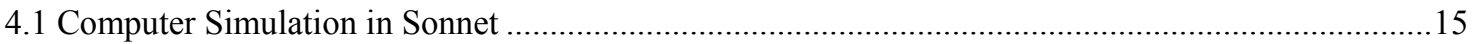

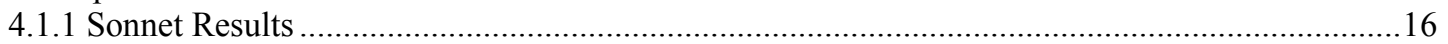

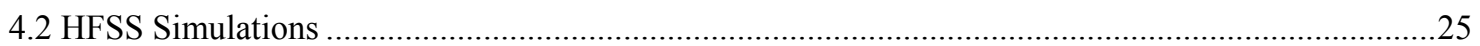

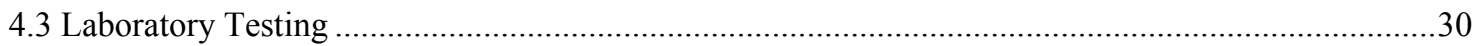

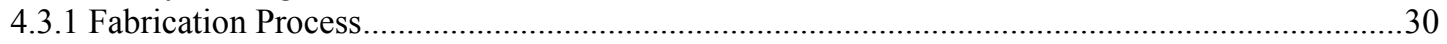

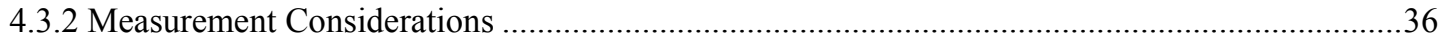

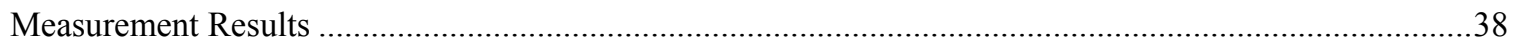

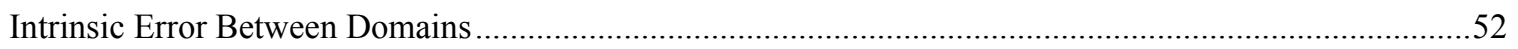

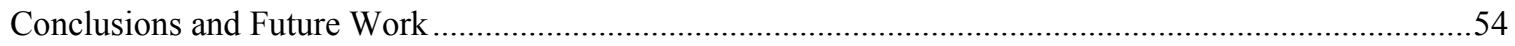

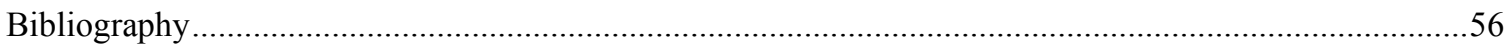

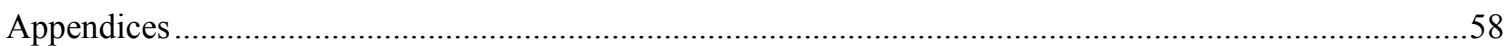




\section{LIST OF FIGURES}

FIGURES

PAGE

Figure 1. Microstrip (a), Stripline (b), and Microstrip with dielectric overlay (c) ……................................2

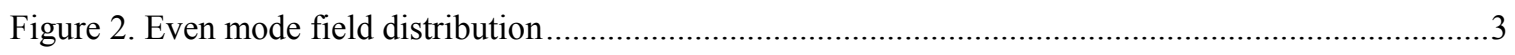

Figure 3. Potential distributions for $\mathrm{x} \& \mathrm{y}$ directions in even mode...........................................................

Figure 4. Effective field distribution for even mode ...............................................................................

Figure 5. Equivalent self capacitances and mutual inductances for the even mode .......................................5

Figure 6. Center active odd mode field distribution ..........................................................................

Figure 7. Potential distributions for $\mathrm{x} \& \mathrm{y}$ directions for center active odd mode........................................6

Figure 8. Self and mutual capacitances for the center active odd mode......................................................

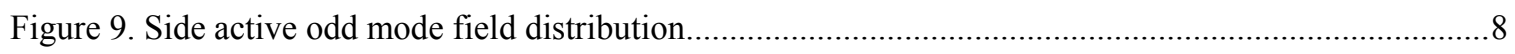

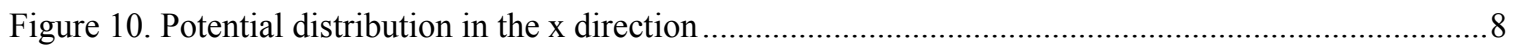

Figure 11. Equivalent self and mutual capacitances for the side active odd mode........................................9

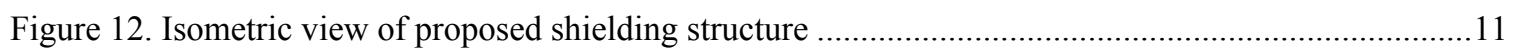

Figure 13. Field distribution for shielded microstrip structure propagating in odd mode ............................12

Figure 14. xy projection of shielded microstrip structure with equivalent self and mutual capacitances......12

Figure 15. Structure/circuit hybrid diagram to illustrate port numbering scheme ..........................................16

Figure 16. 2" Microstrip line cross corner voltage transfer characteristic for shielded and unshielded

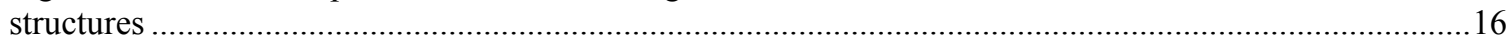

Figure 17. Crosstalk performance of all structures of $50 \Omega$ characteristic impedance …..............................17

Figure 18. Crosstalk performance of all shielded $50 \Omega$ structures............................................................18

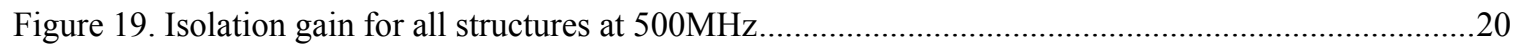

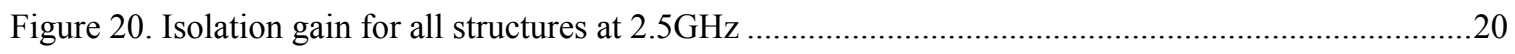

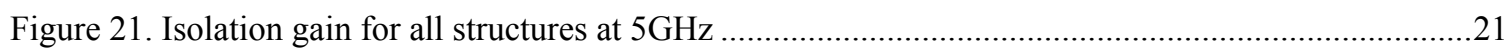

Figure 22. Crosstalk performance of unshielded $50 \Omega$ microstrip for variable thickness ............................21

Figure 23. Crosstalk performance of shielded $50 \Omega$ microstrip on FR-4 of varying thickness ......................22

Figure 24. Crosstalk performance of unshielded $50 \Omega$ microstrip for multiple line lengths .........................22

Figure 25. Crosstalk performance of shielded $50 \Omega$ microstrip for multiple line lengths .............................23 
Figure 26. Crosstalk vs. line spacing for 2" unshielded $50 \Omega$ microstrip ...................................................23

Figure 27. Crosstalk vs. line spacing for 2" shielded $50 \Omega$ microstrip ....................................................24

Figure 28. Land pattern used in the fabrication of test structures............................................................25

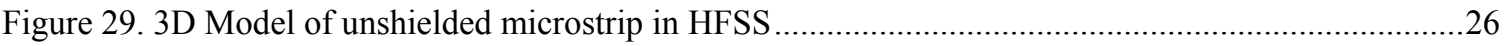

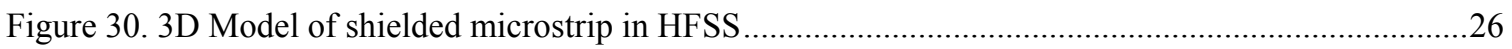

Figure 31. HFSS simulation result for unshielded microstrip vs. frequency ……........................................2

Figure 32. HFSS simulation result for shielded microstrip vs. frequency ………......................................2

Figure 33. HFSS simulation result for unshielded microstrip vs. line spacing.............................................28

Figure 34. HFSS simulation result for shielded microstrip vs. line spacing ...............................................28

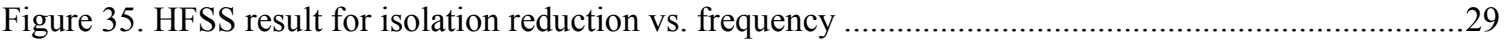

Figure 36. HFSS result for isolation reduction vs. line spacing ............................................................29

Figure 37. Crosstalk performance comparison of $50 \Omega$ microstrip structures ................................................31

Figure 38. AutoCAD drawing used for board fabrication ............................................................................33

Figure 39. Detailed view of a shielded microstrip structure to be fabricated ...............................................34

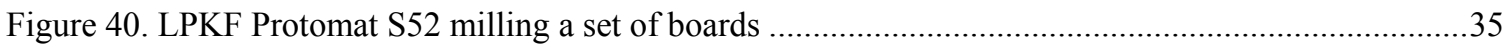

Figure 41. A set of milled boards before contour routing phase ……......................................................35

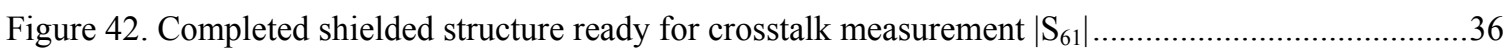

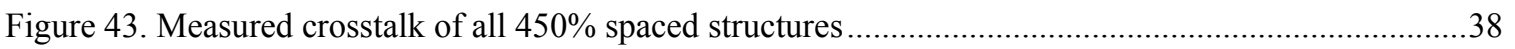

Figure 44. Measured throughput for all 450\% spaced structures .............................................................39

Figure 45. Input reflection coefficient for all $450 \%$ spaced structures.......................................................40

Figure 46. Scattering matrix magnitude for $450 \%$ Line Spacing ............................................................ 41

Figure 47. Three line structure power gain/loss ....................................................................................43

Figure 48. Measured isolation reduction vs. frequency referenced to dual line (ports $3 \& 4$ Terminated) .....45

Figure 49. Measured isolation reduction vs. frequency referenced to three line (ports $3 \& 4$ Terminated) ....45

Figure 50. Measured isolation reduction vs. frequency referenced to dual line (ports $3 \& 4$ open)................46

Figure 51. Measured isolation reduction vs. frequency referenced to three line (ports $3 \& 4$ open) ..............46

Figure 52. Measured isolation reduction vs. frequency referenced to dual line (port 3 open) .......................47 
Figure 53. Measured isolation reduction vs. frequency referenced to three line (port 3 open) 47

Figure 54. Measured isolation reduction vs. frequency referenced to dual line (port 4 open) ....................48

Figure 55. Measured isolation reduction vs. frequency referenced to three line (port 4 open) ..................48

Figure 56. Measured isolation reduction vs. line spacing referenced to dual line structure $(\mathrm{f}=500 \mathrm{MHz}) \ldots . .49$

Figure 57. Measured isolation reduction vs. line spacing referenced to three line structure $(\mathrm{f}=500 \mathrm{MHz}) \ldots .49$

Figure 58. Measured isolation reduction vs. line spacing referenced to dual line structure $(\mathrm{f}=2.5 \mathrm{GHz}) \ldots . .50$

Figure 59. Measured isolation reduction vs. line spacing referenced to three line structure $(\mathrm{f}=2.5 \mathrm{GHz}) \ldots . .50$

Figure 60. Measured isolation reduction vs. line spacing referenced to dual line structure $(\mathrm{f}=5.0 \mathrm{GHz}) \ldots . .51$

Figure 61. Measured isolation reduction vs. line spacing referenced to three line structure $(\mathrm{f}=5.0 \mathrm{GHz}) \ldots . .51$

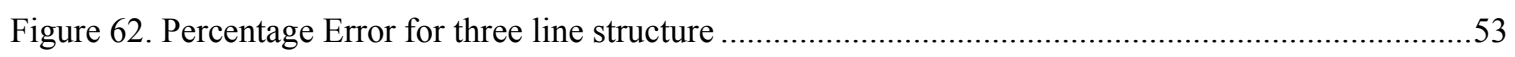

Figure 63. Percentage Error for terminated shielded structure ............................................................53 


\section{Chapter 1}

\section{INTRODUCTION}

Modern mobile communication handsets, computer motherboards, graphics cards circuit boards, and PCI interconnects all utilize of parallel transmission lines that transmit digital signals between components. For transmission lines carrying different information electromagnetic coupling between these lines is of a destructive nature. All devices suffer reduced signal integrity because of this coupling. This corruption is due to capacitive and/or inductive coupling which was studied extensively by $[\mathbf{1}, \mathbf{2}, \mathbf{3}, \mathbf{4}, \mathbf{5}, \mathbf{6}, \mathbf{7 , 8 , 9}, \mathbf{1 0}]$.

The focus of this study is on narrow side coupling of parallel stripline, microstrip and microstrip with dielectric overlay transmission lines on FR-4 fiberglass epoxy substrate. In particular, this study focuses on the characterization of three symmetric coupled transmission lines and an experimental two line shielded geometry. Scattering parameters are numerically calculated, using finite element method solvers, from each structure while varying the physical dimensions of each structure. This thesis topic was suggested by Peter Bartels of Motorola and the idea for shielding the lines is accredited to Dr. Grover Larkins. Mr. Bartels prediction and reason for concern is such that data buses of the future will operate at frequencies between $500 \mathrm{MHz}$ and $5 \mathrm{GHz}$ where inter-line coupling becomes problematic. The primary objective of the thesis is to predict signal corruption between the transmission lines in the aforementioned form factors by means of computer simulations with Sonnet and HFSS. The validation of the simulated results will be accomplished by fabrication of the microstrip transmission lines and testing on a vector network analyzer.

The results of this study will contribute to the design of parallel data buses by allowing layout engineers of printed circuit boards $(\mathrm{PCB})$ to accurately predict signal corruption before running extensive and timeconsuming simulations. Designers would be able to refer to information listed in this manuscript or adopt the methodology described in the following chapters to arrive at a model for their selected substrate because $\varepsilon_{\mathrm{r}}$ is fixed for this study. 


\section{CHAPTER 2}

\section{Coupled Planar Transmission line Structures}

Planar transmission lines such as stripline and microstrip have the ability to propagate transverse electromagnetic (TEM) waves and quasi TEM waves, respectively. The solutions to the line parameters are non-trivial and the procedure of obtaining the line parameters involves the application of partial differential equations which yield solutions that involve infinite summations for the capacitance [7] within the dielectric medium separating the conductive planes and strips. The focus of this research is not on the analysis of a single line which was done in [11], but on the analysis of 2 and 3 narrow side coupled lines in stripline, microstrip and microstrip with a dielectric overlay.

a.

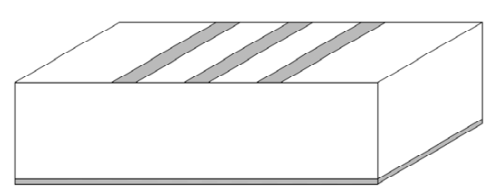

b.

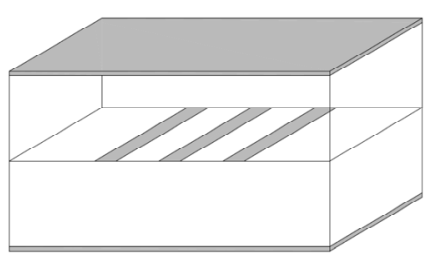

c.

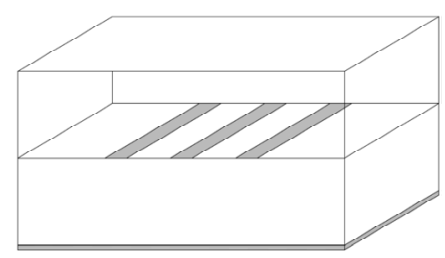

Figure 1. Microstrip (a), Stripline (b), and Microstrip with dielectric overlay (c)

The analysis of coupled lines is logically more complex than that of a single line. Many engineers and scientists have developed different methods to analyze $\mathrm{N}$-coupled transmission line structures but the route to a nominal solution for all of these methods is through numerical techniques where certain simplifications have been made $[\mathbf{3 , 4}]$ due to mathematical complexity.

\subsection{Field Visualization}

To establish a simplified concept of the coupling mechanisms involved for the different propagation modes of three coupled transmission lines begin by finding find orthogonal components of fields at different points of the cross sectional structure. This implies that we are assuming a TEM propagation mode for all 3 modes. For a three coupled line structure there are three modes of propagation that we define as the side active odd mode, center active odd mode, and the even mode. Even mode propagation is defined as all three signals propagating in the same direction. The center active odd mode is defined as the center conducting strip propagating in the $\pm \mathrm{z}$-direction and the outer strips propagating signals in the $\mp$ z-direction. 
The side active odd mode is defined as either the left or right strip propagating in the $\pm \mathbf{z}$-direction and the center and right or left in the $\mp$ z-directions.

\subsection{EVEn Mode Field ANALysis}

Assume a signal with amplitude $\mathrm{V}(\mathrm{z})=+\mathrm{V}_{0} \mathrm{e}^{-\mathrm{j} \beta \mathrm{z}}$ is applied to all lines in the microstrip geometry shown in the figure $(\mathrm{XX})$ below and that the lines are terminated with matched loads to eliminate reflected waves.

The potential distribution in the $\mathrm{x}$-direction at $\mathrm{y}=\mathrm{b}$ is given by the curve below along the potential distribution in the $y$-direction under each strip. That is $\mathrm{x}=[0, \mathrm{~W}],[\mathrm{W}+\mathrm{S}, 2 \mathrm{~W}+\mathrm{S}],[2(\mathrm{~W}+\mathrm{S}), 3 \mathrm{~W}+2 \mathrm{~S}]$.

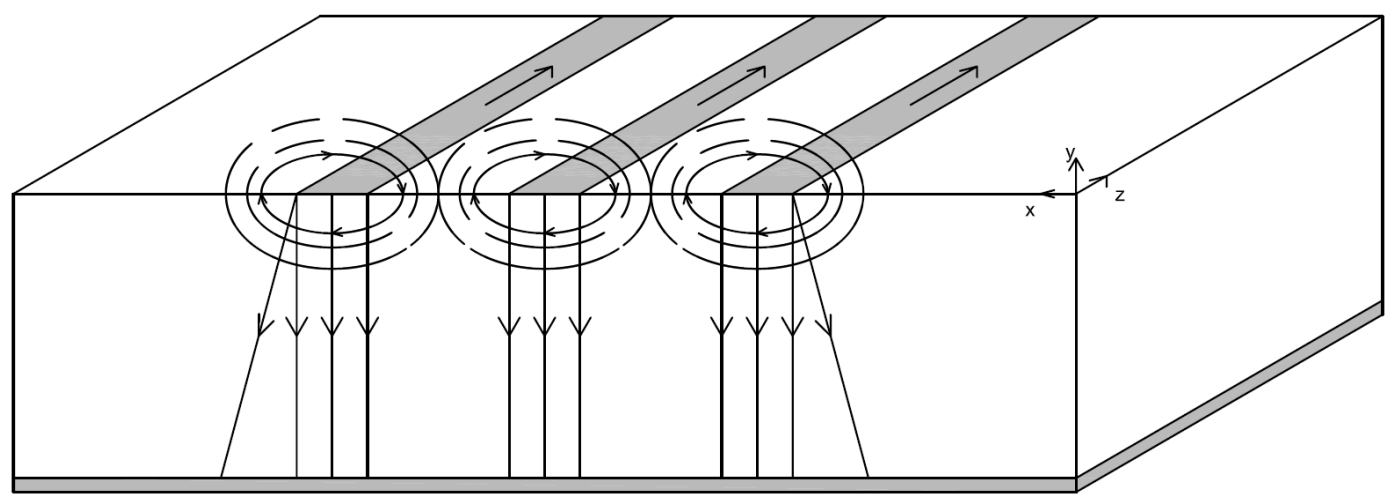

Figure 2. Even mode field distribution
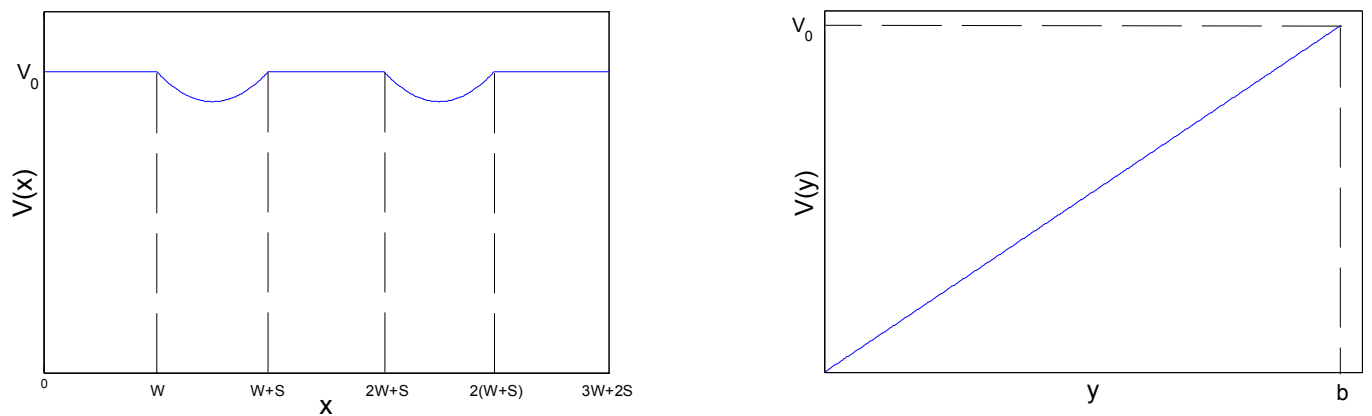

Figure 3. Potential distributions for $x \& y$ directions in even mode

The dips in $\mathrm{V}(\mathrm{x})$ represent the drop in potential between the transmission lines Assuming a sloped change in potential between the strips at $\mathrm{y}=\mathrm{b}$ and finding the $\mathrm{x}$-component of the electric field by:

$$
\vec{E}=-\nabla V
$$


We can see that the electric $\mathrm{x}$ components oppose each other and that there is no electric field component between the strips in the even mode. The y-component of the magnetic field can now be found by using the following equation.

$$
\begin{array}{ccc}
\overrightarrow{H_{y}}=\frac{1}{\eta} \hat{z} \times \overrightarrow{E_{x}} & \\
\overrightarrow{E_{x}}>0 & \text { for } x=\left[W, W+\frac{S}{2}\right] \&\left[2 W+S, 2 W+\frac{S}{2}\right] & \overrightarrow{H_{y}}<0 \\
\overrightarrow{E_{x}}<0 \quad \text { for } x=\left[W+\frac{S}{2}, W+S\right] \&\left[2 W+\frac{S}{2}, 2(W+S)\right] & \overrightarrow{H_{y}}>0
\end{array}
$$

The wave impedance of the TEM wave propagating on the line, $\eta$, is only dependent on the material properties. The magnetic field y-components are in opposite directions in the left and right half of the separation between the lines. By a similar procedure we can calculate the x-component of the magnetic field between the ground plane and the strip conductors.

$$
\begin{gathered}
\overrightarrow{H_{x}}=\frac{1}{\eta} \hat{z} \times \overrightarrow{E_{y}} \\
\overrightarrow{E_{y}}<0 \quad \text { for } x=[0, W] ;[W+S, 2 W+S] ;[2(W+S), 3 W+2 S]
\end{gathered}
$$

The above result shows that the $\mathrm{x}$-component of the magnetic field under each line is in the same direction and thus they add to each other. Combining these results with those of the y-component of the magnetic field clearly shows that there is a net magnetic field encircling all 3 transmission lines.

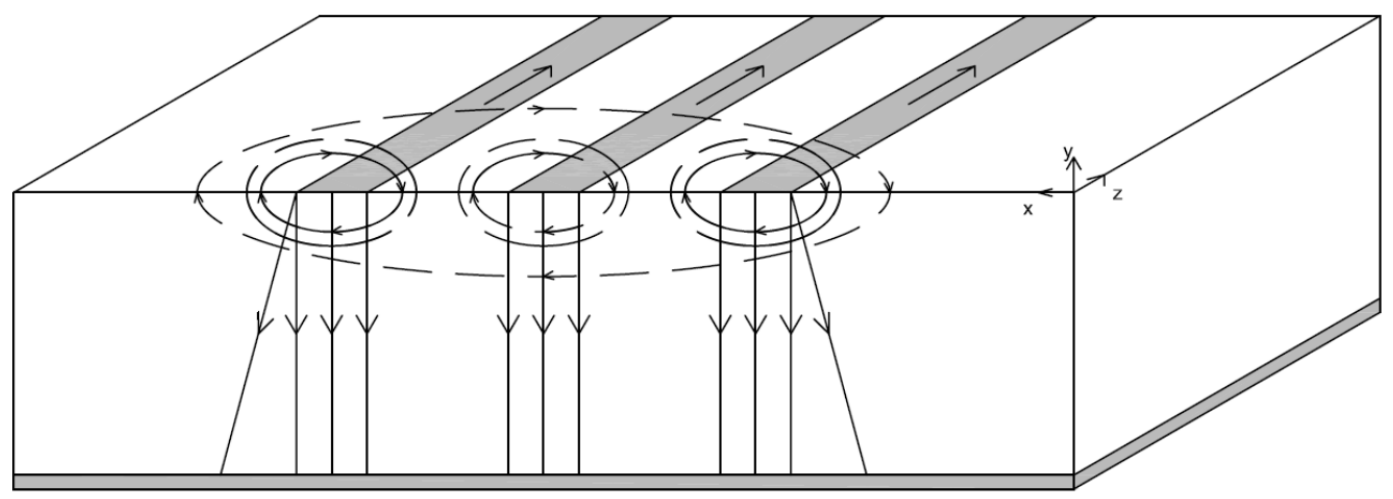

Figure 4. Effective field distribution for even mode 
Each transmission line forms a closed loop with the ground plane. The x-component of the magnetic field and the surface normal of the loop give rise to a mutual inductance between each line on top from their own self-inductance.

$$
\begin{gathered}
\Phi_{i j}=\int_{S_{j}} \overrightarrow{B_{1}} \cdot d \vec{s} \\
L_{i j}=\frac{\Phi_{i j}}{I_{i}} \\
{[\boldsymbol{L}]=\left[\begin{array}{lll}
L_{11} & L_{12} & L_{13} \\
L_{21} & L_{22} & L_{23} \\
L_{31} & L_{32} & L_{33}
\end{array}\right]}
\end{gathered}
$$

$\Phi_{i j}$ is the magnetic flux linkage between lines $i$ and $j . L_{i i}$ represents the self-inductance of the $i$ th line and $L_{i j}$ represents the mutual inductance between the $i$ th and $j$ th lines. From the symmetry in the structure it is clear that the inductance matrix is diagonally symmetric. Capacitive coupling between transmission lines is not a large concern in the even mode because the x-components of the electric fields are of equal magnitude and opposite orientation.

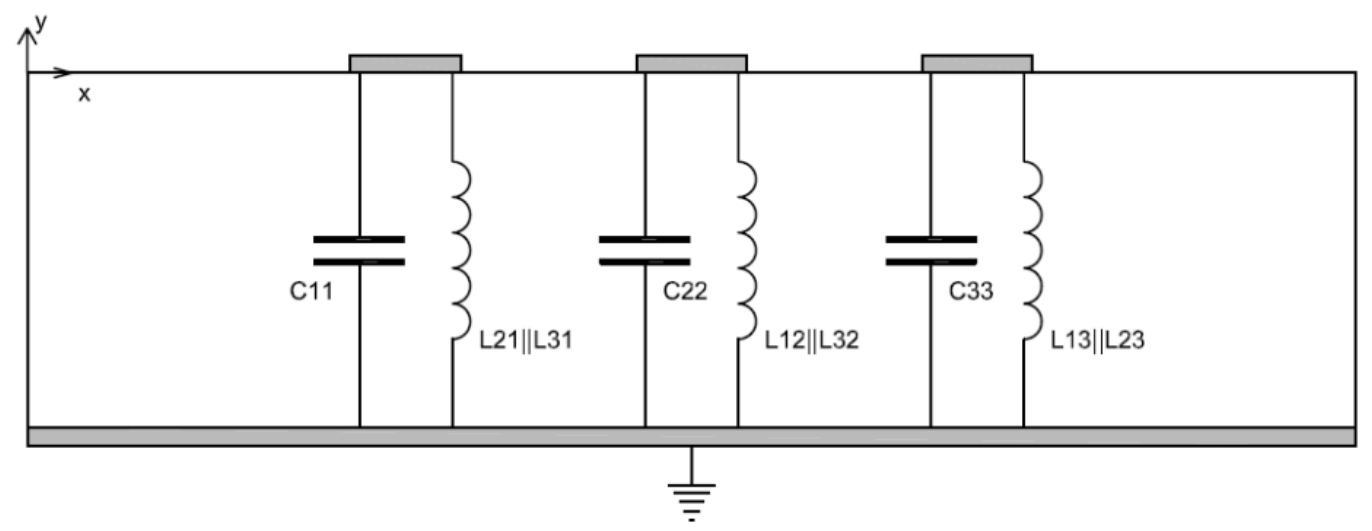

Figure 5. Equivalent self capacitances and mutual inductances for the even mode

\subsection{Center ACtive Odd Mode Field AnAlysis}

The center active odd mode has the two side transmission lines propagating in the direction opposite to that of the center transmission line. In contrast to the even mode, capacitive coupling will be dominant due to 
the potential difference between the conducting strips. The continuous electric field lines from the center conductor to the side conductors imply that the vector sum of the magnetic fields between the lines equals zero because of the opposing propagation directions.

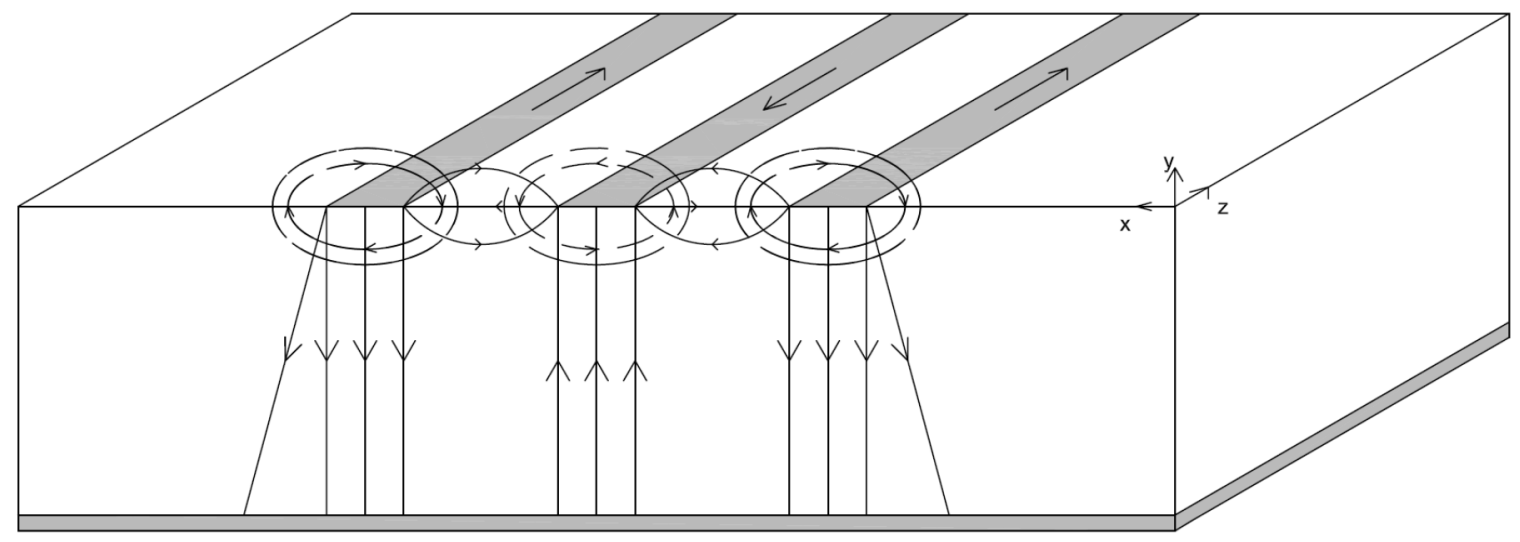

Figure 6. Center active odd mode field distribution

The center conductor has a signal propagating in the +z-direction and the outer conductors in the $-\mathrm{z}$ direction. By a similar analysis we can approximate the potential distributions in the $\mathrm{x}$ and $\mathrm{y}$ directions and follow the same procedure that will lead to the determination of the directions of electromagnetic fields.

a.

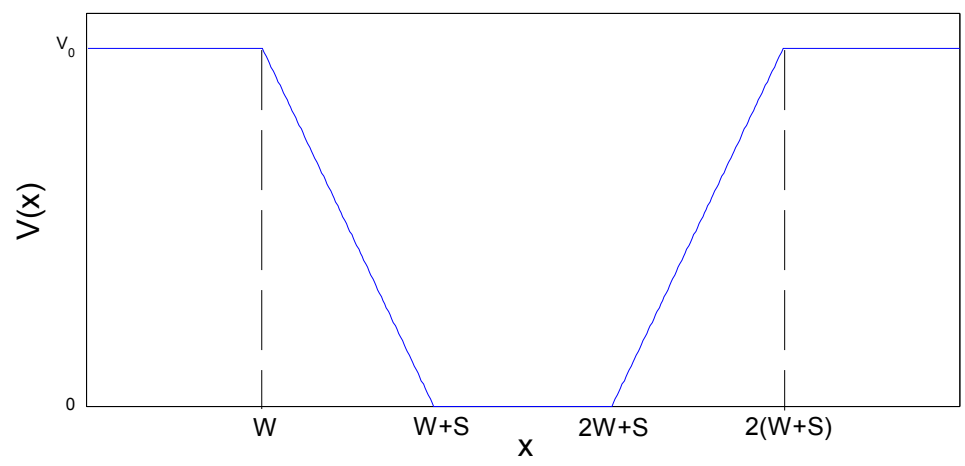

b.

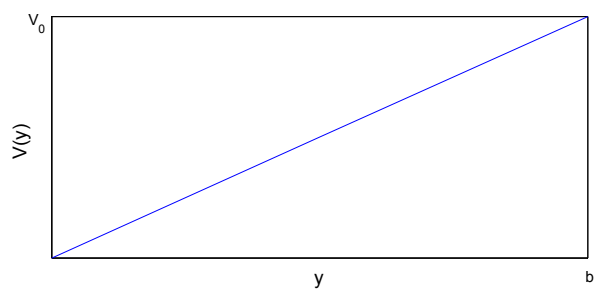

c.

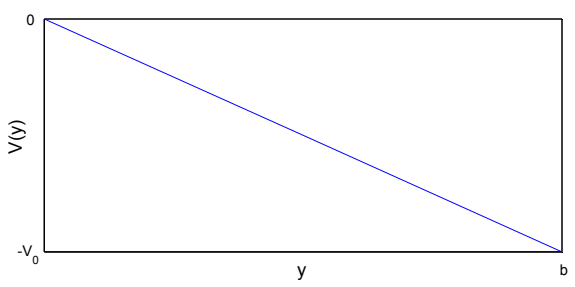

Figure 7. Potential distributions for $x \& y$ directions for center active odd mode 
The potential distribution for $\mathrm{x}=[\mathrm{W}, \mathrm{W}+\mathrm{S}]$ gives rise to an electric field oriented in the $+\mathrm{x}$-direction and the potential distribution for $\mathrm{x}=[2 \mathrm{~W}+\mathrm{S}, 2(\mathrm{~W}+\mathrm{S})]$ gives an electric field component oriented along the $-\mathrm{x}-$ component. For $\mathrm{x}=[\mathrm{W}, \mathrm{W}+\mathrm{S} / 2]$ there exists a magnetic field $\mathrm{y}$-component aligned with the $-\mathrm{y}$-direction due to the signal propagating on the left conductor, and for $\mathrm{x}=[\mathrm{W}+\mathrm{S} / 2, \mathrm{~W}+\mathrm{S}]$ the $\mathrm{y}$-component of the magnetic field is in the -y-direction as well. A similar trend can be found from $x=2 W+S$ to $x=2(W+S)$. These adding magnetic fields do not contribute to mutual inductance because they are orthogonal to the surface normal of the loop made by each strip conductor and the ground $(\mathrm{B} \cdot \mathrm{ds}=0)$.

The potential distribution along the y-direction underneath the left and right transmission lines gives rise to an electric field oriented in the -y-direction which implies that the x-component of the magnetic field is in the $-\mathrm{x}$-direction. For the center line, the electric field is oriented in the $+\mathrm{y}$-direction and yields a magnetic field $\mathrm{x}$-component oriented along the $+\mathrm{x}$-direction. The fact that the $\mathrm{x}$-components of the magnetic fields between conductors are canceled out means that the network is magnetically decoupled. The potential difference between the center conductor and the left and right conductors gives an electric field component that is inversely proportional to the spacing between them. This leads to the conclusion that capacitive coupling between the lines in the center active odd mode is the dominant force in destructive coupling for the mode. The capacitive network distribution for the mode is illustrated in the figure below.

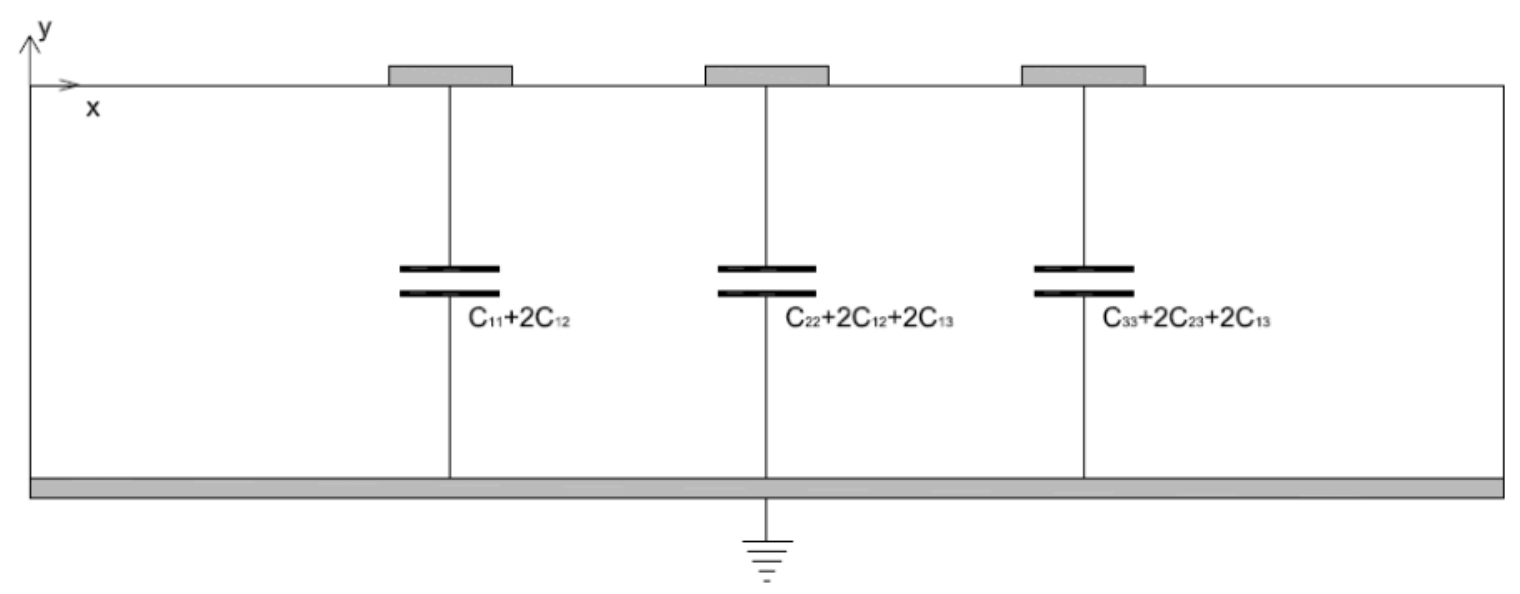

Figure 8. Self and mutual capacitances for the center active odd mode 


\subsection{Side Active Odd Mode Field Analysis}

For the final mode of propagation we will assume that the leftmost strip conductor is propagating a TEM wave in the $+\mathrm{z}$-direction and the center and right conductors are propagating a signal in the $-\mathrm{z}$-direction. The potential distribution in the parameter for $\mathrm{y}=\mathrm{b}$ is shown below.

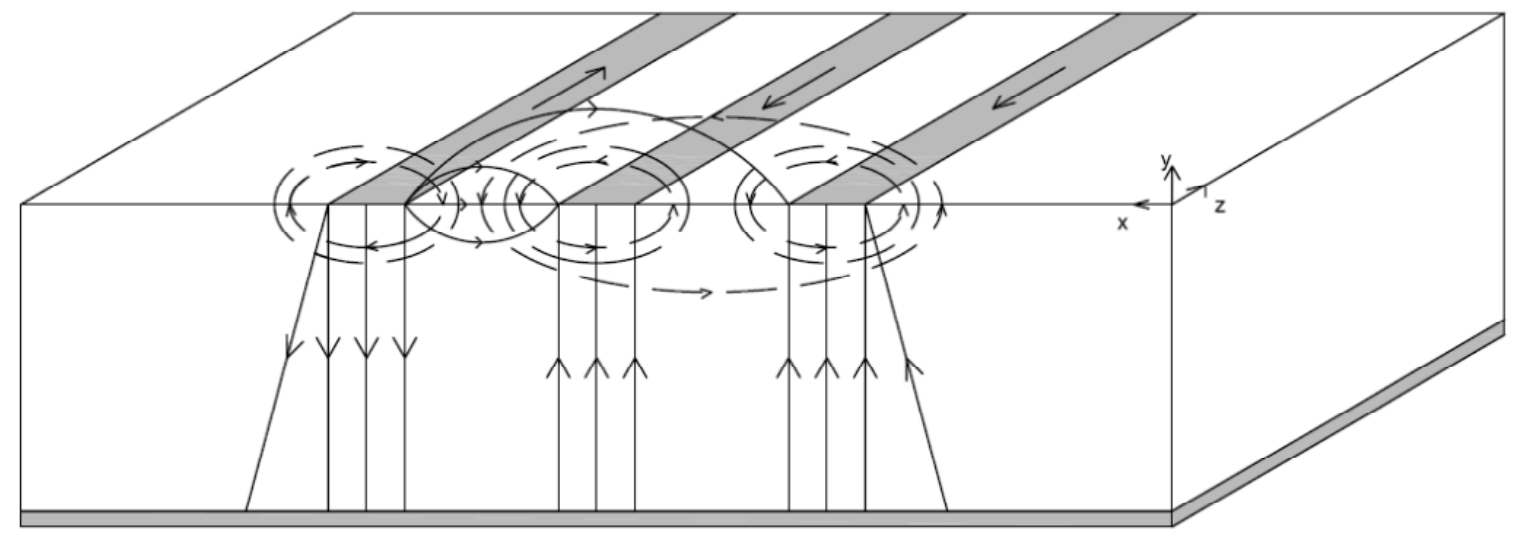

Figure 9. Side active odd mode field distribution

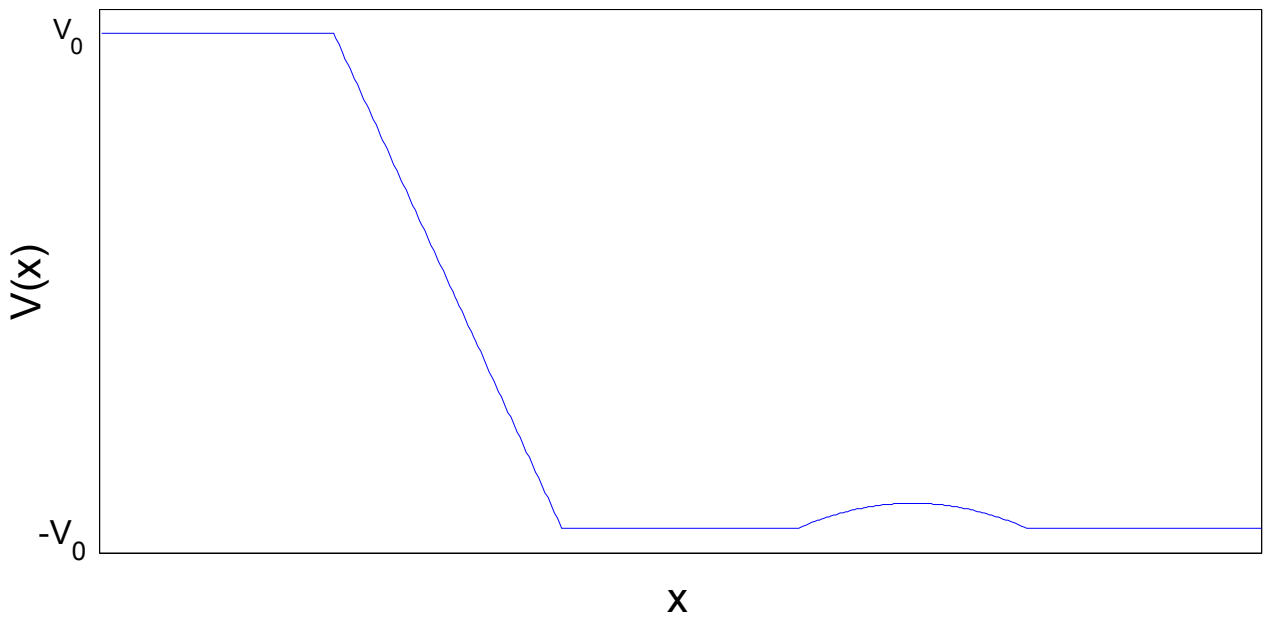

Figure 10. Potential distribution in the $x$ direction 
The potential distribution between the left and center lines is clearly analogous to that of the center active odd mode. The potential distribution between the right and center conductors is similar to that for the even mode described earlier. Referring to the results of the center active odd mode it is evident that capacitive coupling will dominate for the separation of the left and center transmission lines. Inductive coupling will be dominant mechanism of signal corruption in the separation between the center and right conductors. In contrast to the other two modes of signal transmission, this mode gives rise to the greatest amount of field asymmetry and, as a result, makes analysis more complicated. An equivalent circuit of the network capacitances is shown below.

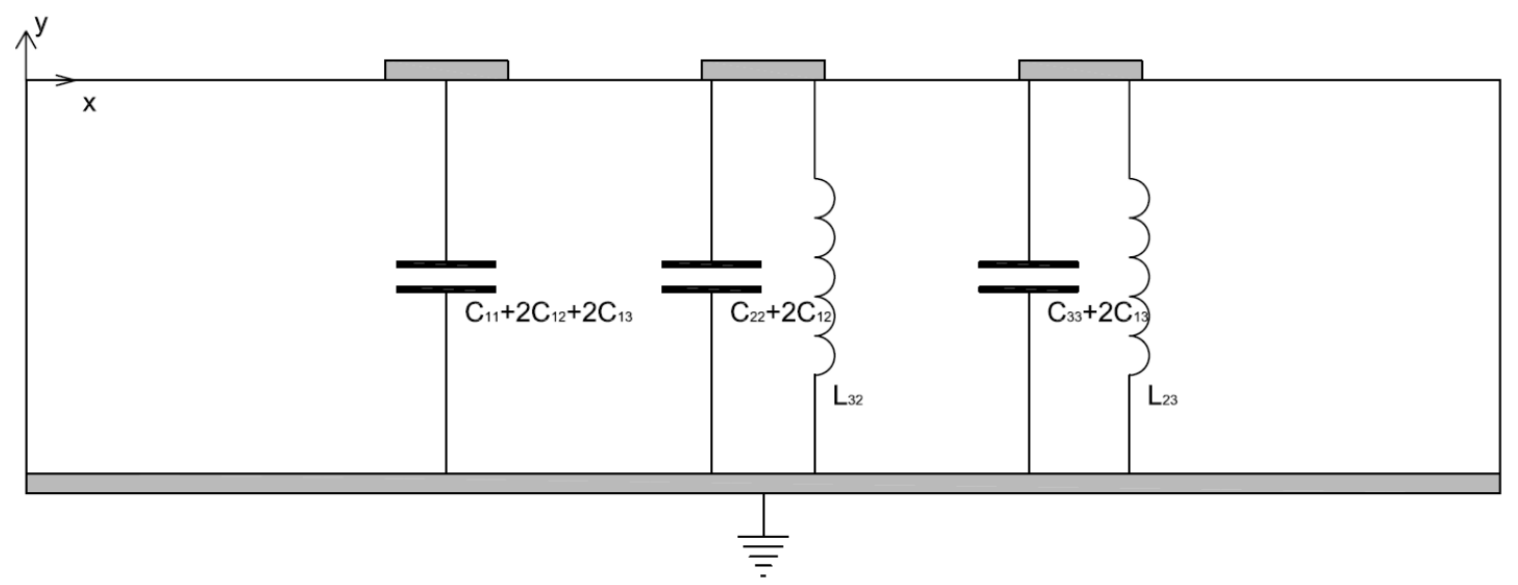

Figure 11. Equivalent self and mutual capacitances for the side active odd mode

\subsection{QUantitative ANalysis}

Some researchers have studied crosstalk in microstrip using even and odd mode analysis [1,7], others have analyzed the mutual inductances and capacitances $[\mathbf{2 , 3 , 4 , 5 , 6}]$. Zhou [12] cited Leone $[\mathbf{1 3}]$ stating that certain approximations and assumptions were made that ignore the line width and field distribution, not because he was inadequate but because, "It is difficult to obtain solution with both simplicity and accuracy if one just studies the coupling in a direct way [12]." Abbosh [10] confirms Zhou [12] by stating, "Analytical studies result in closed-form expressions which are suitable for analysis and design, but the accuracies depend to a large extent on the involved approximations." 
Abbosh goes on to say that numerical methods conducted by $[\mathbf{1 4 , 1 5}]$ are accurate but do not yield practical algorithms for design optimization. Integral equations utilizing image theory were performed by Hellman and Palocz [16], and they pointed out that conformal mapping was performed on a single microstrip by Black and Higgins [17] and the resulting integral cannot be evaluated in closed form when applied to multiple microstrip lines. 


\section{CHAPTER 3}

\section{ENHANCING NOISE IMMUNITY}

One proposed solution to reduce electromagnetic coupling between parallel lines is to have a grounded conducting strip between the transmitting lines. This grounded center strip is terminated into a matched load on both ends, and has periodically spaced of vias along the length of the conductor. The purpose of these vias along with the center conductor is to capture radiated electric fields and create a closed surface for magnetic fields to permeate and cancel based of the principle of Faraday's Law. This work will examine the even and odd propagating modes in this structure give a qualitative analysis of the field behavior of this structure and explain how it acts to reduce the coupling between lines.

\subsection{Odd Mode Propagation}

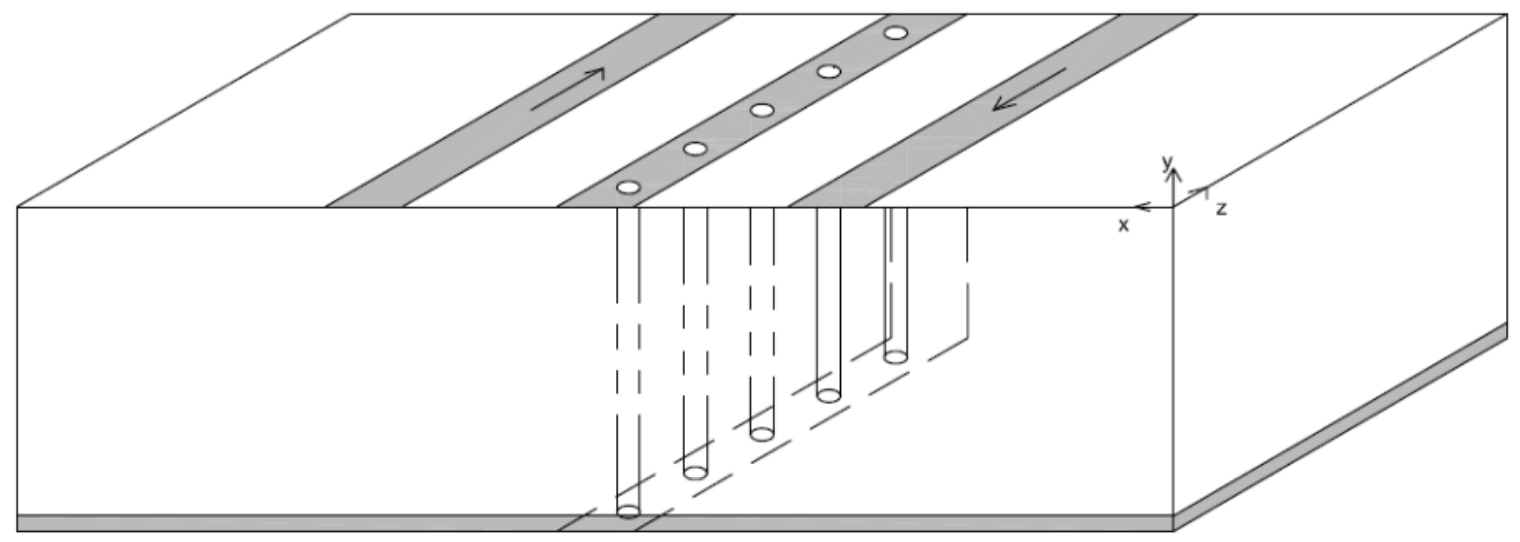

Figure 12. Isometric view of proposed shielding structure

Capacitive coupling is the dominant mechanism that leads to signal degradation for signals propagating in opposite directions. The insertion of a grounded strip conductor with periodic short circuit through holes allows parasitic electric fields to couple to this conductor and dissipate into the ground plane. For two signals propagating in opposite directions with their voltage maximums $180^{\circ}$ out of phase, their electric fields are such that they couple to the center conductor and the vias. The electric fields couple to the center conductor and cancel due to induced surface charge. Recalling the potential distributions in the $\mathrm{x}$-direction for the center active odd mode it is easy to see that there is a potential gradient which induces an electric 
field inversely proportional to the spacing of the lines and directly proportional to the potential difference between the lines.

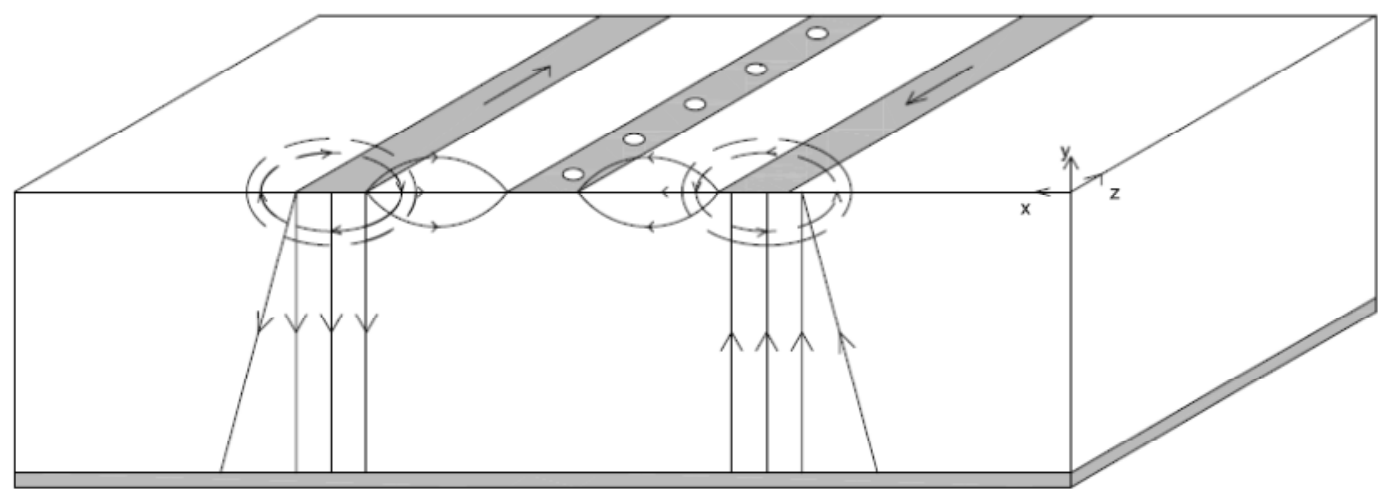

Figure 13. Field distribution for shielded microstrip structure propagating in odd mode

Grounding the center conductor allows the potential distribution in the x-direction to have a smaller slope for the same spacing. This smaller slope means a lower magnitude $\mathrm{x}$-oriented electric field. The fact that the coupling is now with a grounded conductor ensures that there is less re-radiation. This in turn reduces the corruption from the signal on its neighboring transmission line. The grounded conductor serves as a channel for the radiated energy.

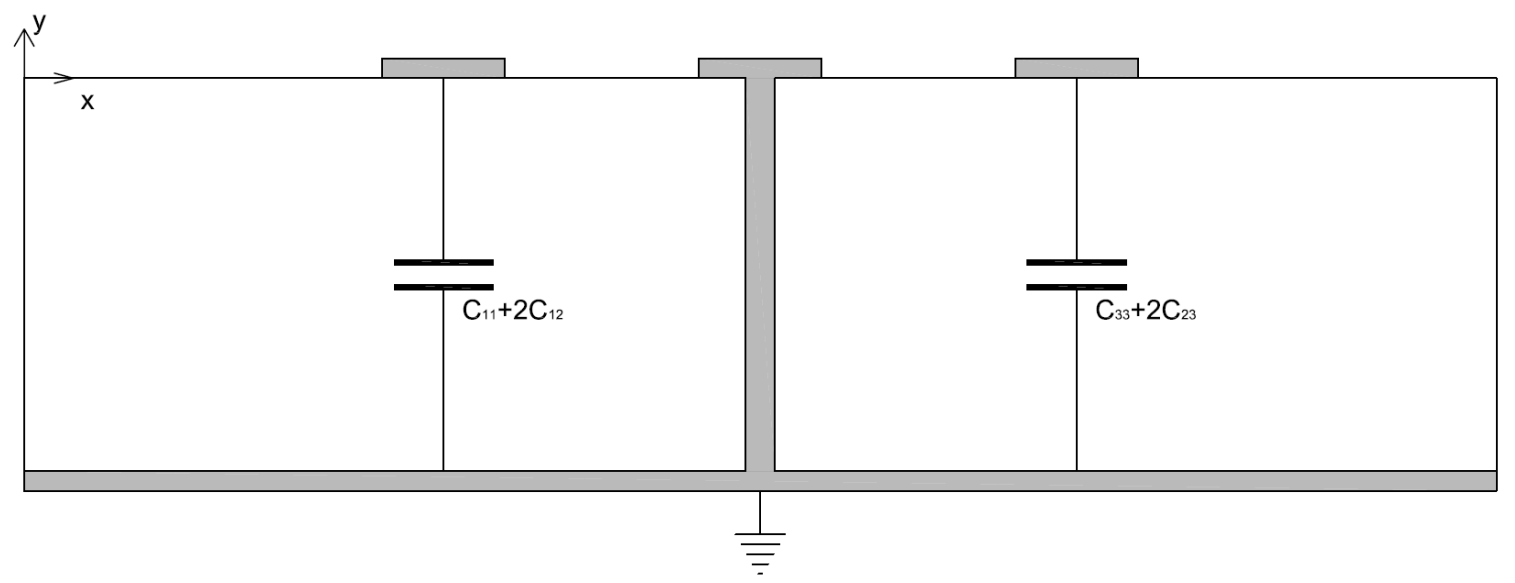

Figure 14. xy projection of shielded microstrip structure with equivalent self and mutual capacitances

Magnetic fields will be circulating in opposite directions which tell us that the vertical components will sum up and create stronger fields but fall off by inverse distance squared. However, horizontal components 
oppose each other. Assuming symmetrical conditions and identical signals propagating such that peak amplitude of one signal and minimum amplitude of the other are aligned along a plane $\mathrm{z}=\mathrm{z}_{0}$ such that $\mathrm{z}_{0}$ is less than the length of the line, we can conclude that the horizontal components of the magnetic fields between the lines will completely cancel.

\subsection{Even Mode Propagation}

The even mode propagation for the shielded lines has a similar mechanism to enhance noise immunity with the exception being that it comes from magnetic fields. Again for TEM wave propagation we will have magnetic fields permeating the surfaces of the loops created in the center conductor and through hole shorts. These magnetic fields, according to Faraday's Law will induce eddy currents into these loops.

$$
V_{e m f}=-\frac{d \Phi}{d t}=-\int_{S} \frac{d \vec{B}}{d t} \cdot d \vec{s}
$$

The time derivative can be moved into the integrand because the area of the surface is not changing in time. Utilizing Kirchoff's Current Law, we can see that the currents will sum in such a way as to not cancel each other. These induced currents will in turn generate magnetic fields to oppose the magnetic fields penetrating the closed surface of the loop thus enhancing the noise immunity of one line from the other.

In order for induced magnetic field to cancel the incident magnetic field, the self-inductance of the shield line must be as high as possible and the resistance of the line as low as possible so that the phase difference between the induced voltage and induced current approaches ninety degrees. One such method to ensure that line inductance is sufficiently high is to make sure that the vias are made with the smallest diameter to height ratio possible as determined by Goldfarb and Pucel [18].

\subsection{ALTERNATIVE MethODS OF CROSSTALK SUPPRESSION}

Other variations to reduce crosstalk between planar transmission line structures have been worked upon and have seen success. The more traditional methods include increased line spacing [19] and using materials that have dielectric constants that approach $\varepsilon_{0}[\mathbf{2 0}]$. These methods are intuitive because if we consider the electric fields as the transport mechanism of energy between lines then if line spacing is 
increased by a factor of two then the electric field strength falls off by a factor of 4 and the power induced by a factor of 16 . Recalling the formula for parallel plate capacitance gives an intuition as to why low relative permittivity substrates have lower crosstalk values. Parallel plate capacitance is linearly proportional to the relative dielectric constant. Sometimes greater line spacing is not an option because of mechanical constraints. Other instances may require a particular substrate and lower values of relative dielectric constant are not available.

Other exotic methods that reduce crosstalk include the use of magnetic-loaded absorber sheets and breaks in the ground plane for microstrip. Drnovsek et al [21] was attempting to determine ways to increase line coupling for the design of $3 \mathrm{~dB}$ couplers and phase shifters using magnetic absorbing sheets placed atop the coupled lines. What worked for Drnovsek is not what is of interest. What did not work for Drnovsek is the more interesting case for this research. A magnetic absorber sheet with a relative permeability of 20 and relative dielectric constant of unity resulted in the lowest values of coupling for their research. Kazerooni et al [22] proposed the implementation of parallel slots or total breaks in the ground plane to reduce crosstalk. His results concluded that a parallel break in the ground plane effectively lowered the mutual inductance and capacitance considerably more than a parallel slot.

Suspending a floating conducting surface above a coupled microstrip structure was implemented by Malisuwan and Ungvichian [23] in order to reduce crosstalk. The height of suspension was varied and their results indicated that the closer they could suspend the line the more the crosstalk was reduced. 


\section{CHAPTER 4}

\section{VALIDATION}

The validation of the aforementioned qualitative analyses is carried out by computer simulation and physical fabrication and testing of the boards themselves, shielded and unshielded. With data from simulation and these experiments we can validate the accuracy by comparison. Accuracy of Sonnet's FEM simulator was evaluated by Oldfield et al. [24].

\subsection{COMPUTER Simulation In SONNET}

Computer simulation was initially done in Sonnet, which is a finite element method electromagnetic simulator where models are drawn in planar 3D. That is, metallization is drawn on planes and layers of dielectrics are added in blocks where their shape is not editable. The following table details what physical and electrical parameters were varied in the Sonnet simulator.

\begin{tabular}{|l|l|l|}
\hline Parameter & Values & Units \\
\hline Length & $0.5,1.0,1.5,2.0$ & Inch \\
\hline Substrate Thickness & $0.015625,0.03125,0.0625$ & Inch \\
\hline Line Spacing & $10,20, \ldots, 100$ & $\%$ Line Width \\
\hline Characteristic Impedances & $25,50,75,100$ & Ohms \\
\hline Frequency & $5 \times 10^{6}$ through $5 \times 10^{9}$ & Hertz \\
\hline
\end{tabular}

Table 1. Summary of all parameters varied for simulations performed

Project files were created for every value of line length and for shielded and unshielded lines. 288

individual project files were created that ran a decade frequency sweep in adaptive band sweep mode and a parameter sweep over line spacing where the values of the line spacing were dictated by the width of the line which depends on the characteristic impedance.

The scattering matrix is calculated from the simulations. From geometry considerations we can see that the matrix is diagonally symmetric. A sample drawing below illustrate port placement so that reference to cells of the scattering matrix become clear. 
The results of the simulations yielded values for $\left|\mathrm{S}_{61}\right|$ such that for $10 \%$ line width spacing, the magnitude of voltage transfer is lower across the entire frequency band of interest!

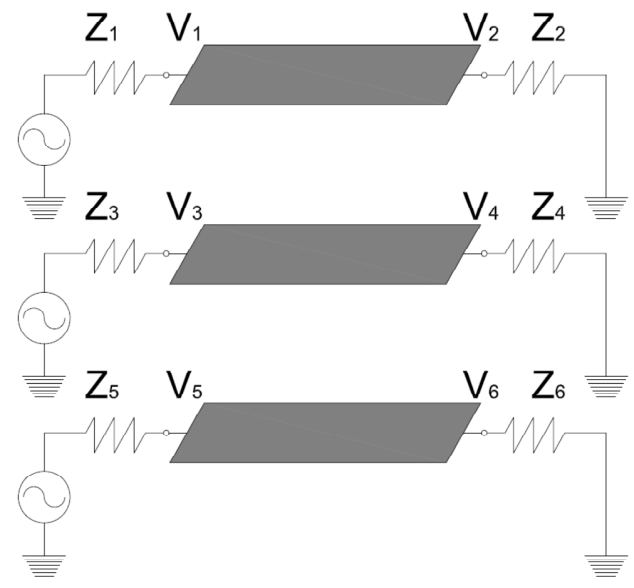

Figure 15. Structure/circuit hybrid diagram to illustrate port numbering scheme

\subsubsection{SONNET RESULTS}

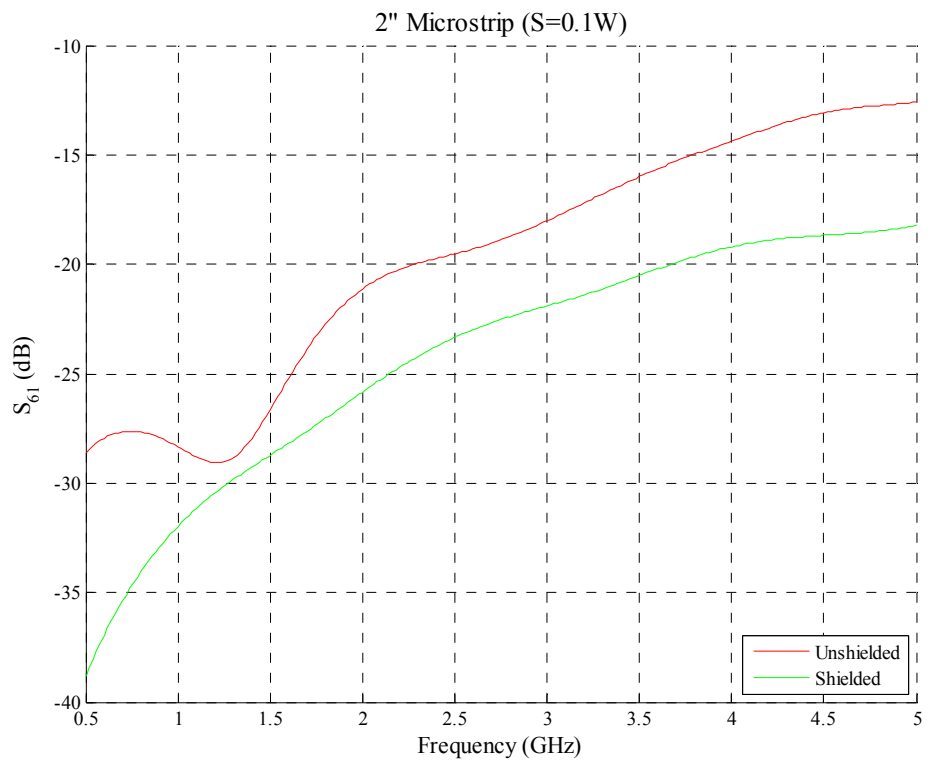

Figure 16. 2" Microstrip line cross corner voltage transfer characteristic for shielded and unshielded structures 
The above figure illustrates the simulation results for $50 \mathrm{Ohm}$ lines on FR-4 epoxy substrate with a 10\% line width spacing $(\mathrm{S}=10.6 \mathrm{mils}=0.26924 \mathrm{~mm})$. At $5 \mathrm{GHz}$, electrical isolation is increased by $5.6403 \mathrm{~dB}$ which corresponds to a $52.24 \%$ reduction of energy transfer when compared to the unshielded case. The various structures under investigation have different coupling characteristics as illustrated below.

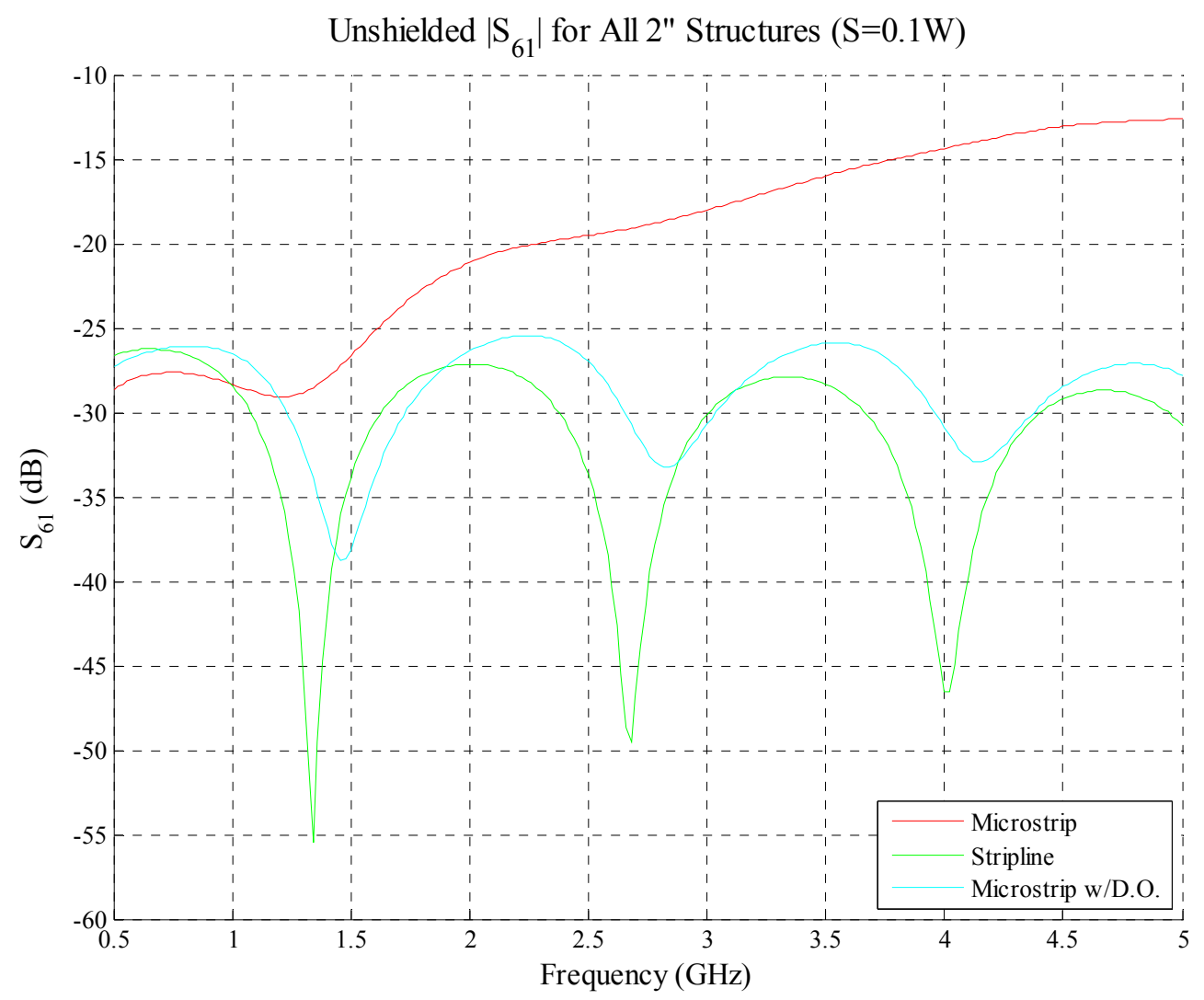

Figure 17. Crosstalk performance of all structures of $50 \Omega$ characteristic impedance

From this plot we can see that the microstrip structure exhibits the most inferior coupling characteristics. When considering the physical layout of the structure, it makes logical sense as to why this characteristic is so. Consider geometric optics and Snell's Law. When there is an electromagnetic wave incident on dielectric media there is a certain angle of diffraction that depends on the ratio of indices of refraction and the sine of the incident angle. The microstrip structure contains only a single ground plane whereas the stripline structure contains dual ground planes. We can postulate that the presence of a second ground plane lowers coupling substantially because the electric fields are more strongly coupled to the ground plane. 
Stripline would closely approximate the characteristics of a coaxial cable because stripline is practically a flattened out coaxial cable where the circular cross sections are stretched into an ellipse and then the major axis of that ellipse approaches an infinite distance to create a rectangular/planar structure. Another observation that can be made is that the resonant frequencies are different for each structure.

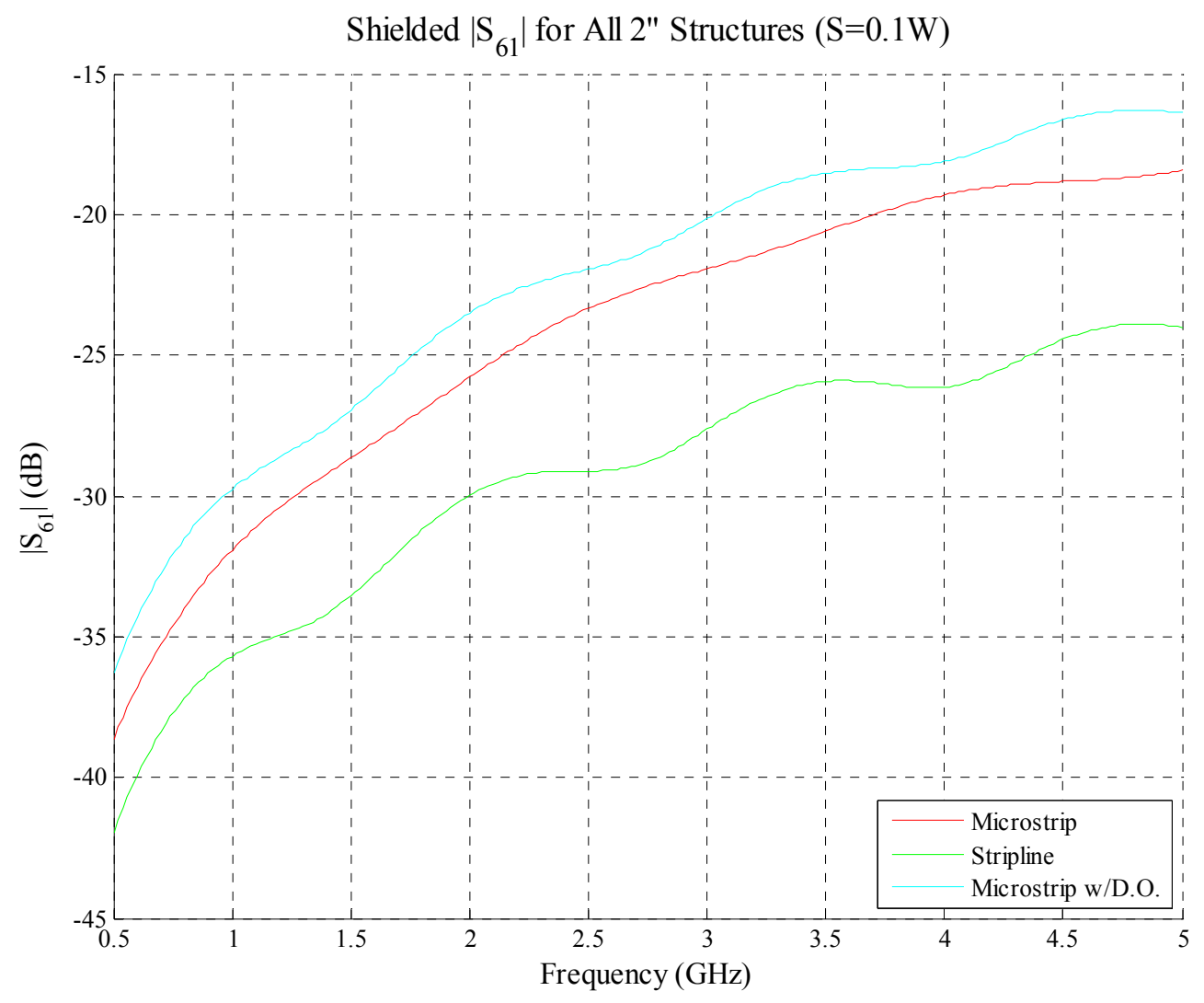

Figure 18. Crosstalk performance of all shielded $50 \Omega$ structures

The microstrip with dielectric overlay is the next step in the progression to the microstrip structure. This structure removes the ground plane thus reducing the strength of the electric field in the vertical directions, i.e. lower strip to ground coupling and increased fringe field strength which leads to higher degree of coupling. There is less diffraction present in this structure because it does not present a discontinuous dielectric interface between strip conductors. Because fields do not diffract here their magnetic fields are better canceled. Therefore spatial dielectric homogeneity is a key factor that could work to designer's advantage when designing high density parallel data buses. 
The microstrip structure, which from the figure above exhibited the worst cross corner coupling characteristics for a structure of the same length and width dimensions suffers this because it has the weakest strip to ground plane electric field coupling. Once again, like the microstrip with dielectric overlay structure, the fringe field coupling to the adjacent conductors is strengthened.

Because the magnitude of the electric field is proportional to potential difference over separation it would make sense that the coupling between lines would decrease for thinner substrates. The figure below illustrates that this is the case at higher frequencies on a microstrip structure fabricated on different substrate thicknesses. Shielded microstrip structures do exhibit the same properties and with greater success. The shielded structure across the entire band of interest exhibits a reduction of corner-to-corner coupling greater than $10 \mathrm{~dB}$. Because all lines are terminated in the same impedance, this would translate into a $20 \mathrm{~dB}$ improvement in signal to noise ratio.

The same way reducing the substrate thickness reduces the magnitude of $S_{61}$ the length of the line also has an effect because the mutual inductance increases with line length because the area of the loop increases proportionally with line length.

Figure 18 shows how the crosstalk performance for stripline and microstrip with dielectric overlay degrade after implementing the shield. In order to view how well the shield is working a new parameter can be defined that quantifies the effectiveness of the shield for a structure, isolation gain or reduction. The isolation gain is defined to be $\left|\mathrm{S}_{61}\right|$ in decibels for an arbitrary unshielded structure minus $\left|\mathrm{S}_{61}\right|$ for its corresponding shielded structure. That is,

$$
\text { IsolationGain }=- \text { IsolationRe duction }=-\left(\left|S_{61}\right|_{\text {Shielded }}-\left|S_{61}\right|_{\text {Unshielded }}\right)(d B)
$$

Figures 19-21 illustrate isolation gain for all structures at $500 \mathrm{MHz}, 2.5 \mathrm{GHz}$ and $5 \mathrm{GHz}$ versus percentage line width spacing. A positive value of isolation gain would indicate that the shield has increased noise immunity by the value indicated at that particular value of line spacing. Negative values of isolation gain would immediately indicate to a designer that the shielded structure would not be worth his/her time to fabricate because the shield would degrade signal integrity between these ports. 
Shielding Effectiveness at $\mathrm{f}=500 \mathrm{MHz}$

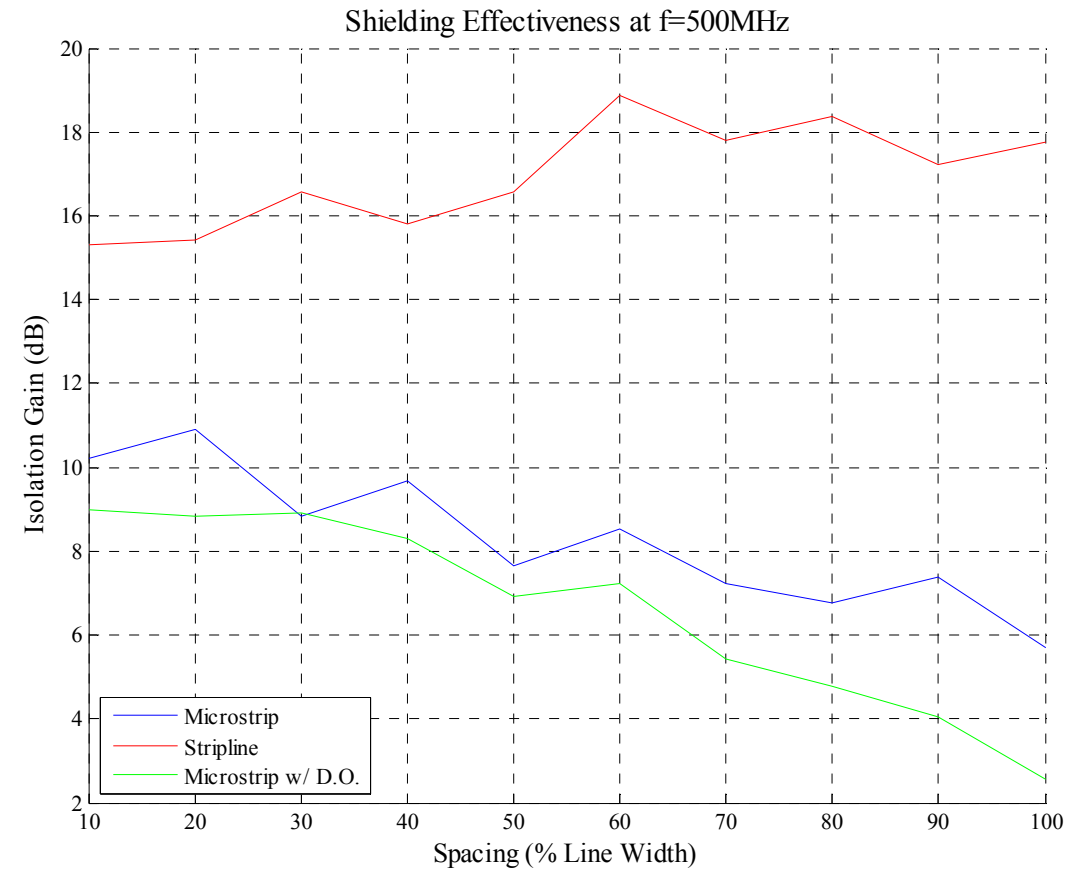

Figure 19. Isolation gain for all structures at $500 \mathrm{MHz}$

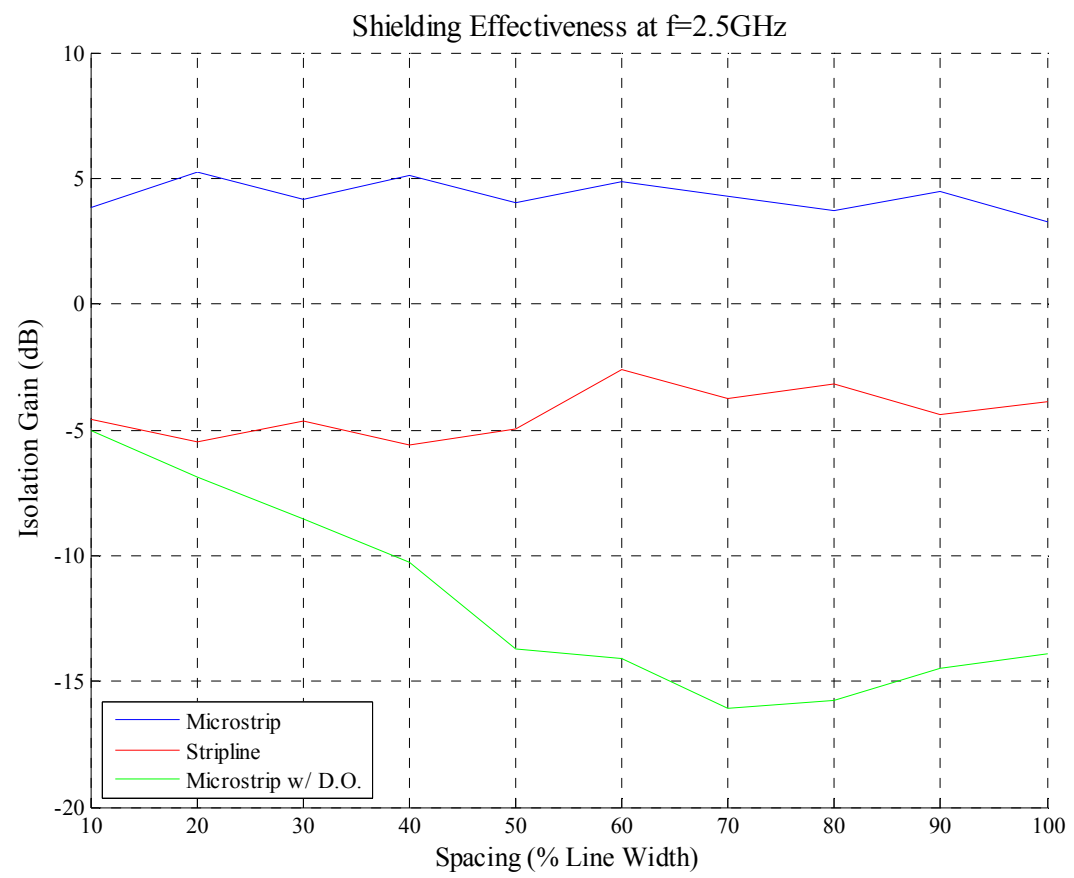

Figure 20. Isolation gain for all structures at $2.5 \mathrm{GHz}$ 


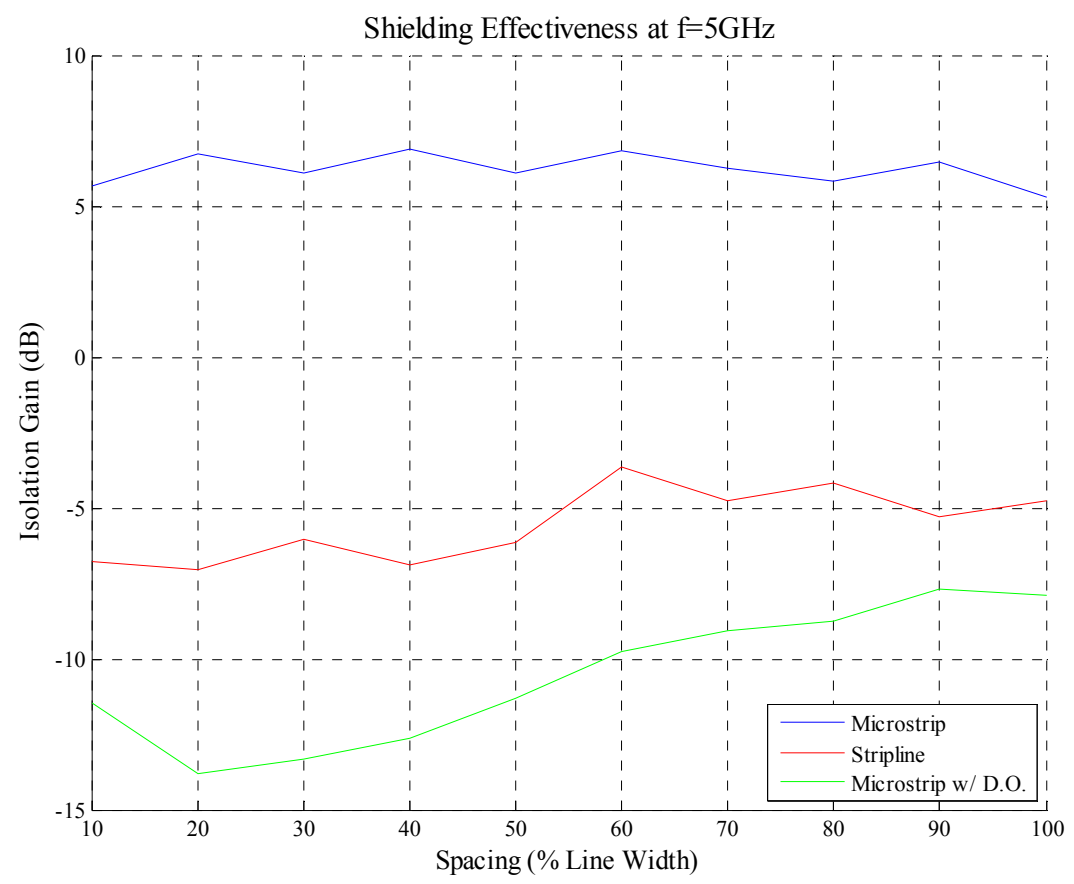

Figure 21. Isolation gain for all structures at $5 \mathrm{GHz}$

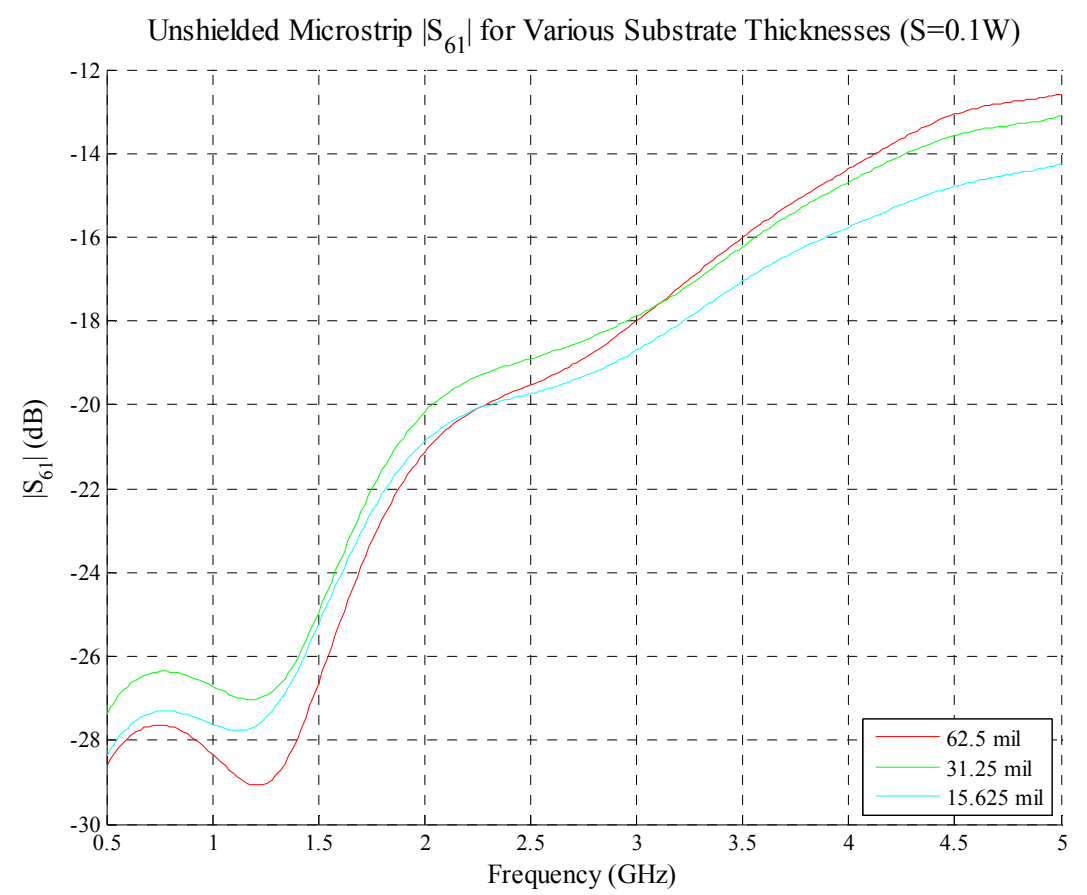

Figure 22. Crosstalk performance of unshielded $50 \Omega$ microstrip for variable thickness 


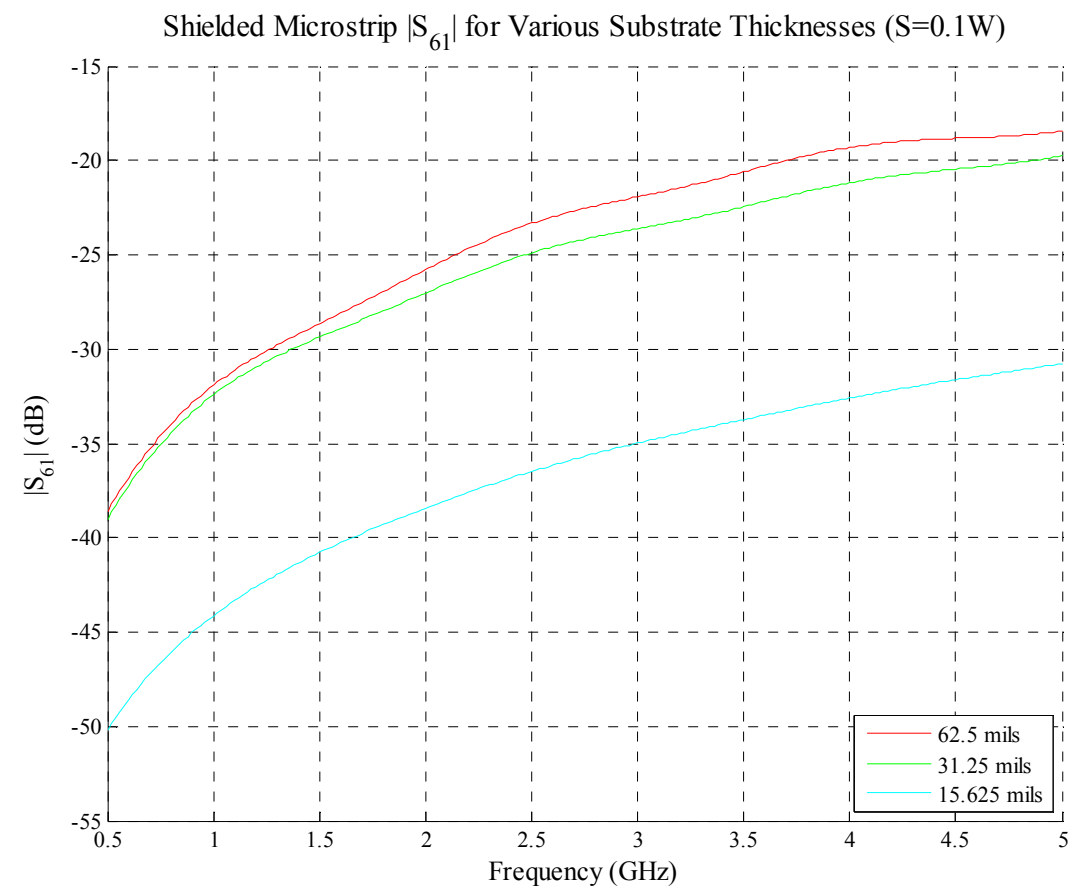

Figure 23. Crosstalk performance of shielded $50 \Omega$ microstrip on FR-4 of varying thickness

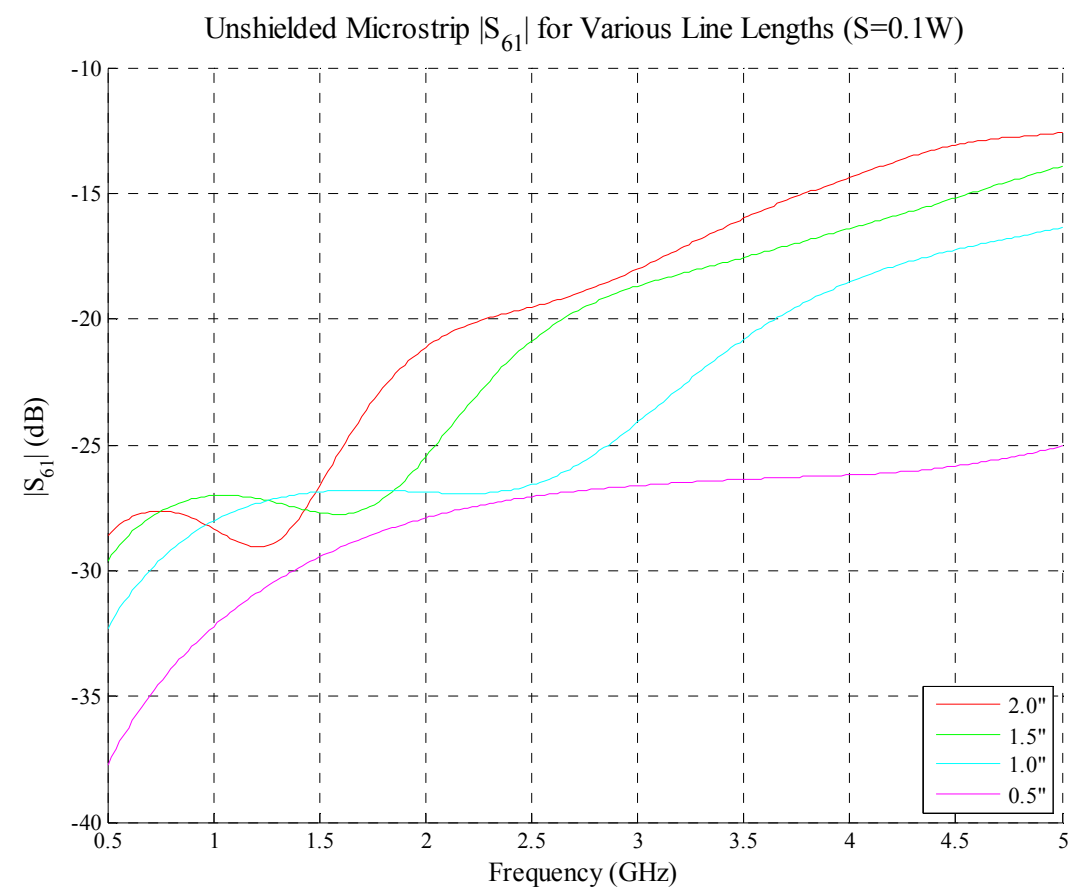

Figure 24. Crosstalk performance of unshielded $50 \Omega$ microstrip for multiple line lengths 


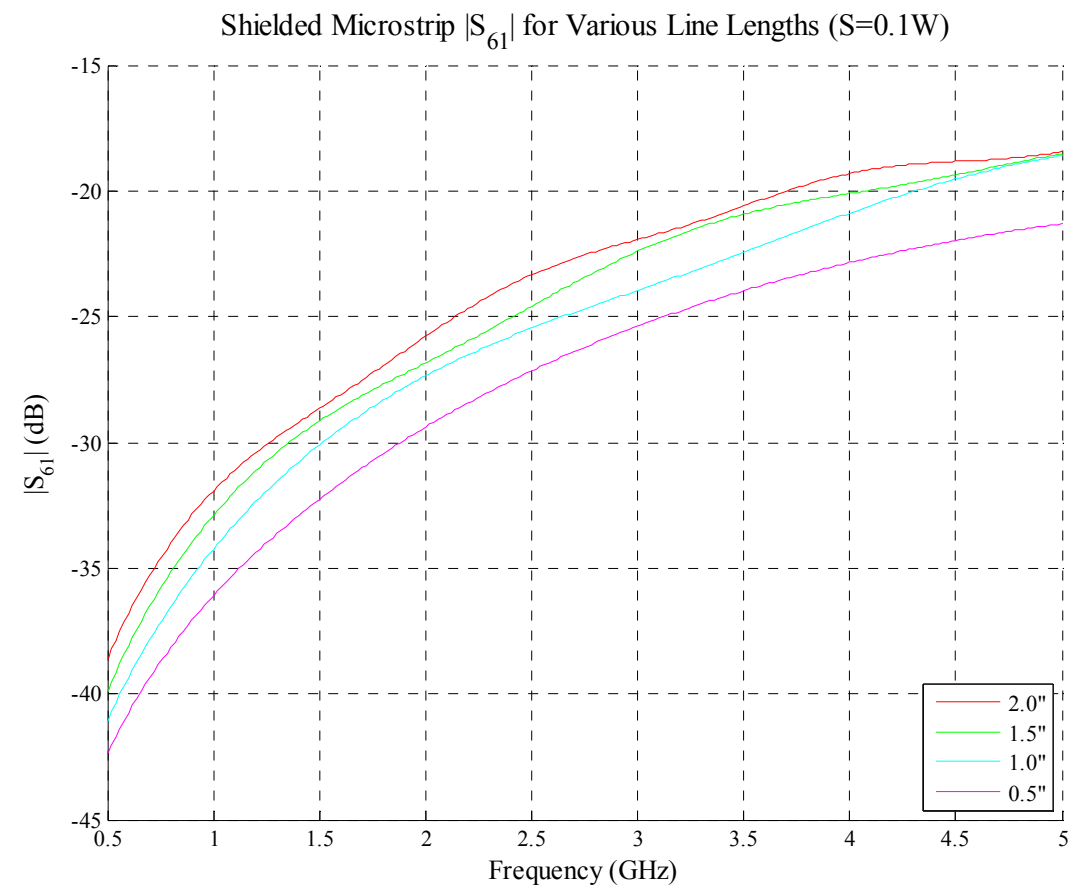

Figure 25. Crosstalk performance of shielded $50 \Omega$ microstrip for multiple line lengths

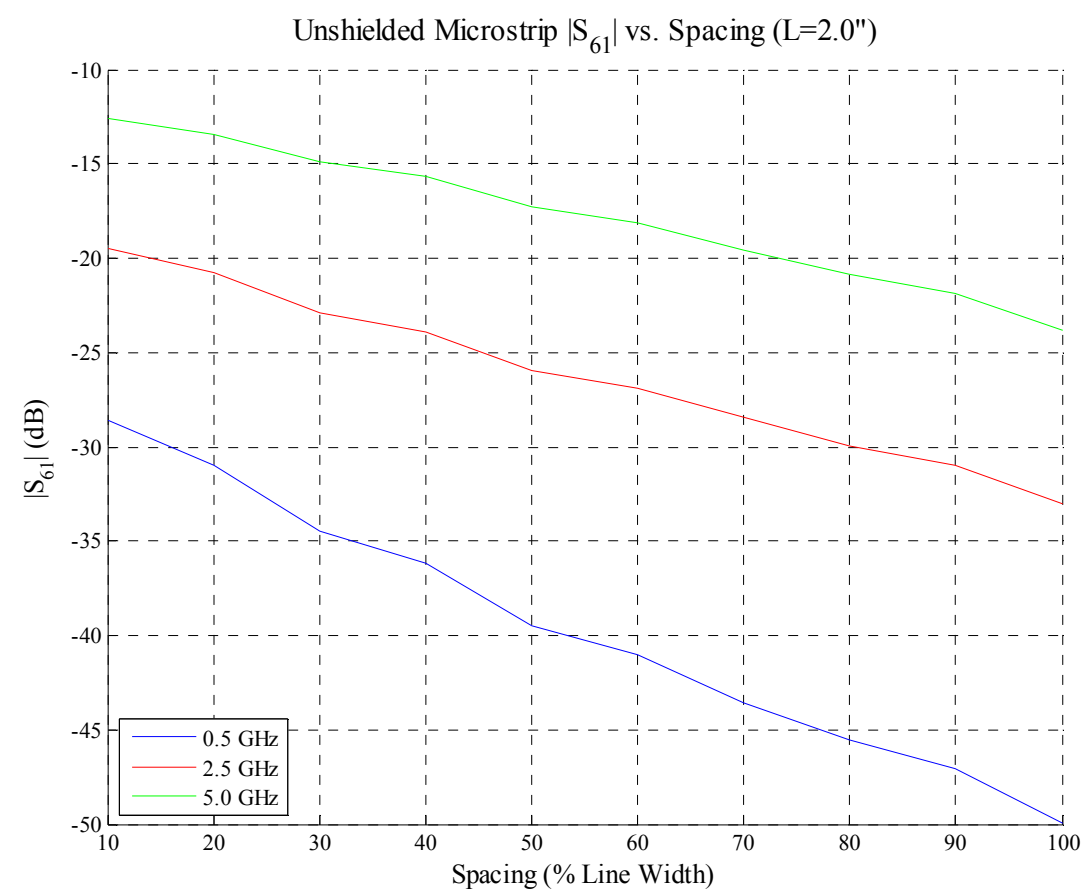

Figure 26. Crosstalk vs. line spacing for 2" unshielded $50 \Omega$ microstrip 


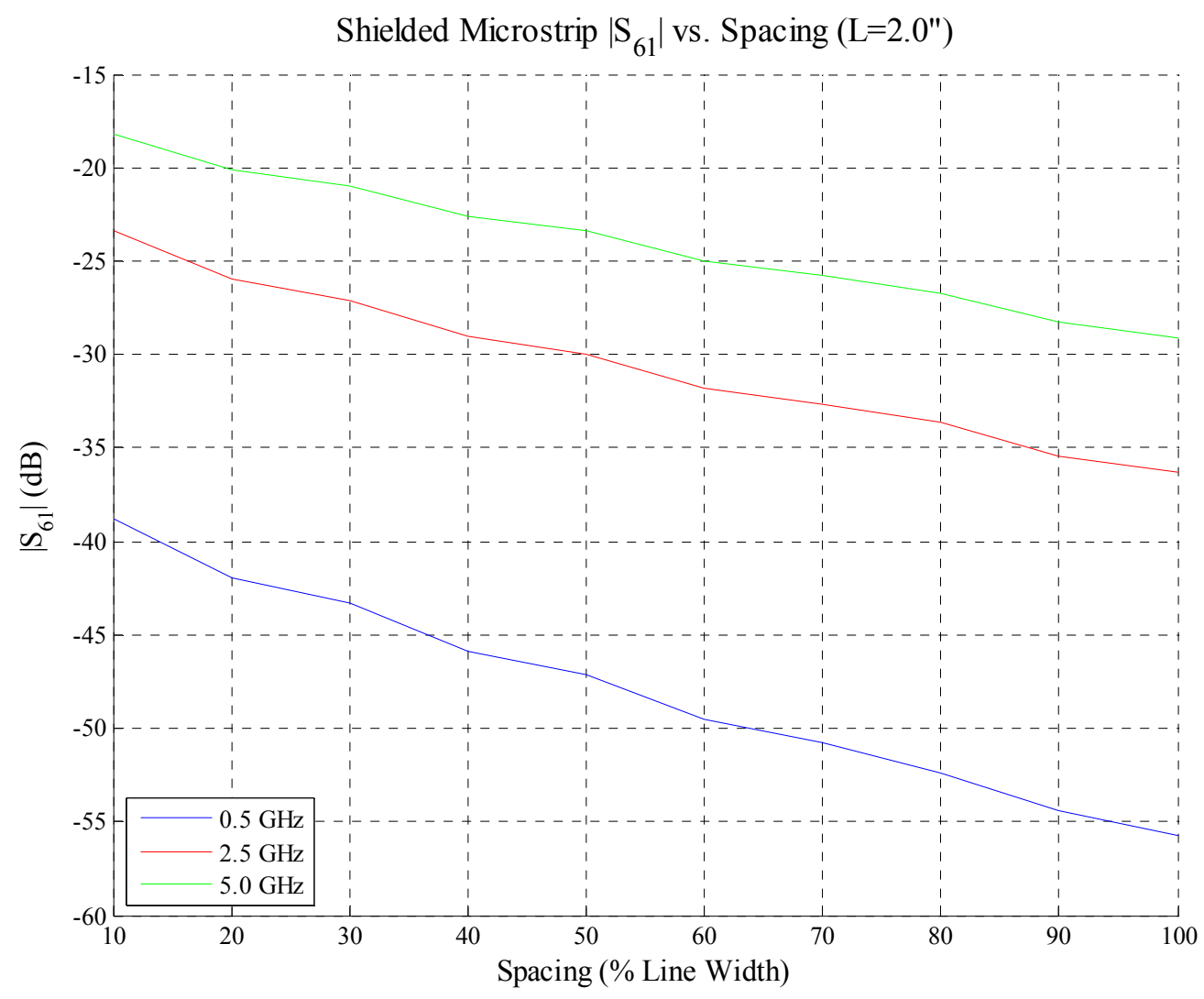

Figure 27. Crosstalk vs. line spacing for 2" shielded $50 \Omega$ microstrip

Seeing how the cross corner coupling changes as the line spacing is increased is a useful way to look at the coupled lines when in a design scenario. The preceding figures illustrate how the crosstalk is reduced as the spacing between the lines is increased for a fixed substrate thickness of $0.0625 "(1.5875 \mathrm{~mm})$.

This view of the curves allows for a clear view of voltage transfer is decreased as the line spacing is increased. This result is in agreement with the results found by Kasuga \& Inoue [19]. The shielded microstrip structure shows a lower amount of crosstalk for the entire spacing range. Where microstrip shows a constant decline in crosstalk for increasing spacing, stripline does not. Stripline and microstrip with dielectric overlay now follow the same trend as microstrip now having lost their periodicity in frequency. To a transmission line designer, this would not be a desirable effect and it is clear that isolation gain is a negative value for these structures. 


\subsection{HFSS SIMULATIONS}

In order to meet VSWR requirements to properly test the structures there is a minimum spacing requirement set by the recommended pattern of the connectors. Improper signal coupling to the board would create spurious radiation at the discontinuities and, in turn, corrupt the measurements. The coaxial to microstrip transition itself is a source of error because it is a line discontinuity. This discontinuity is a measurement error that will discussed in Chapter 6 in greater detail.

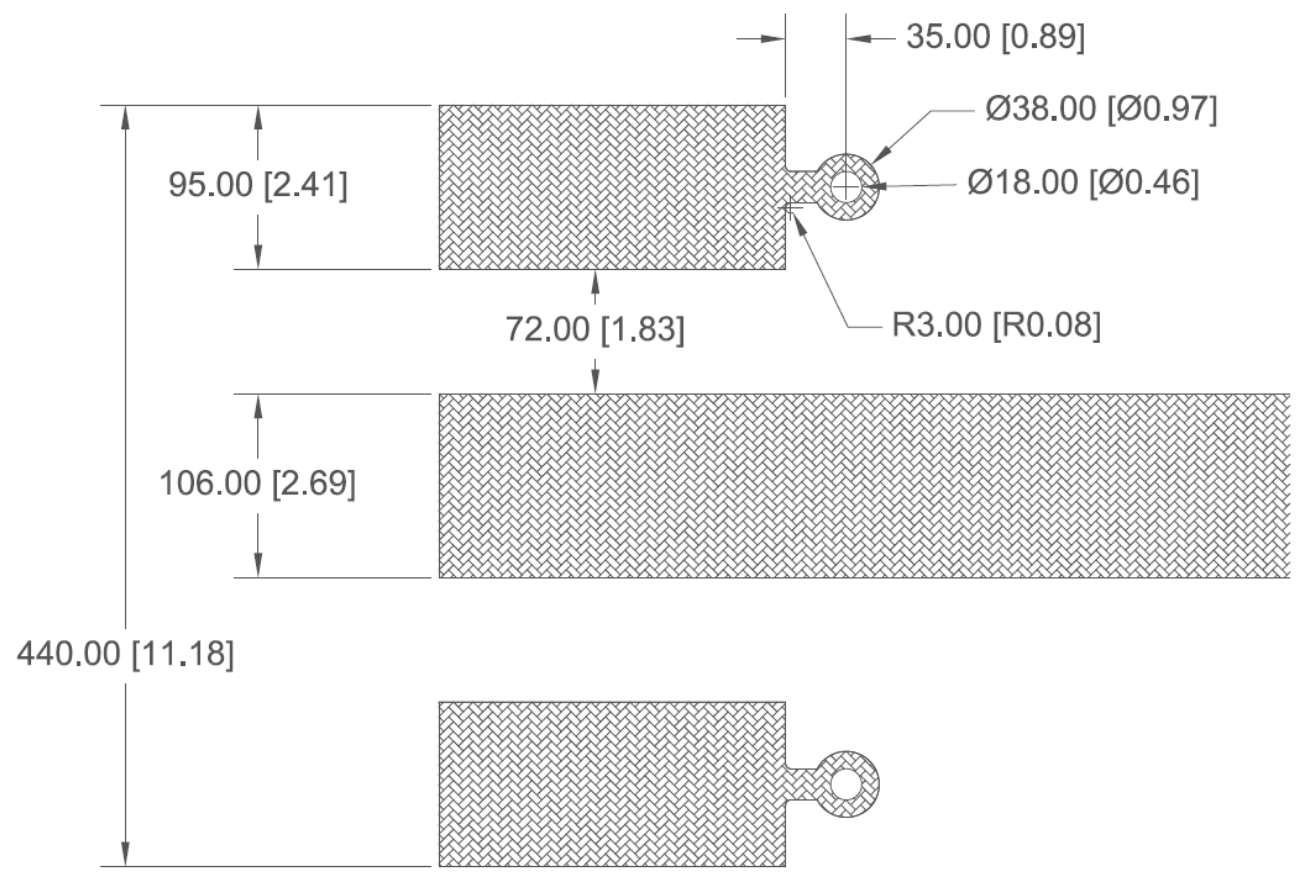

Figure 28. Land pattern used in the fabrication of test structures

The 440 mil width is also the minimum line spacing that imposes the minimum connector spacing. With the minimum value being,

$$
\frac{S_{\min }}{W}=\frac{440 m i l s}{106 m i l s}=4.15
$$

Stepping 4.15 up to 4.5 allows for a small gap between connectors and ensures that connector mounting will be clean and easy. Therefore the minimum value of spacing shifts to $4.5 \cdot \mathrm{W}$ which is equal to $477 \mathrm{mils}$. Stepping these values up 53mils at a time will give the $500 \%, 550 \%$ and $600 \%$ values. This calls for a re- 
simulation of the microstrip structures with the new line spacing values at a fixed length of 2" $(50.8 \mathrm{~mm})$ over the $500 \mathrm{MHz}$ to $5 \mathrm{GHz}$ band. HFSS is used for the re-simulation primarily because of its ability to simulate larger domains where structures are drawn in a full three-dimensional model.

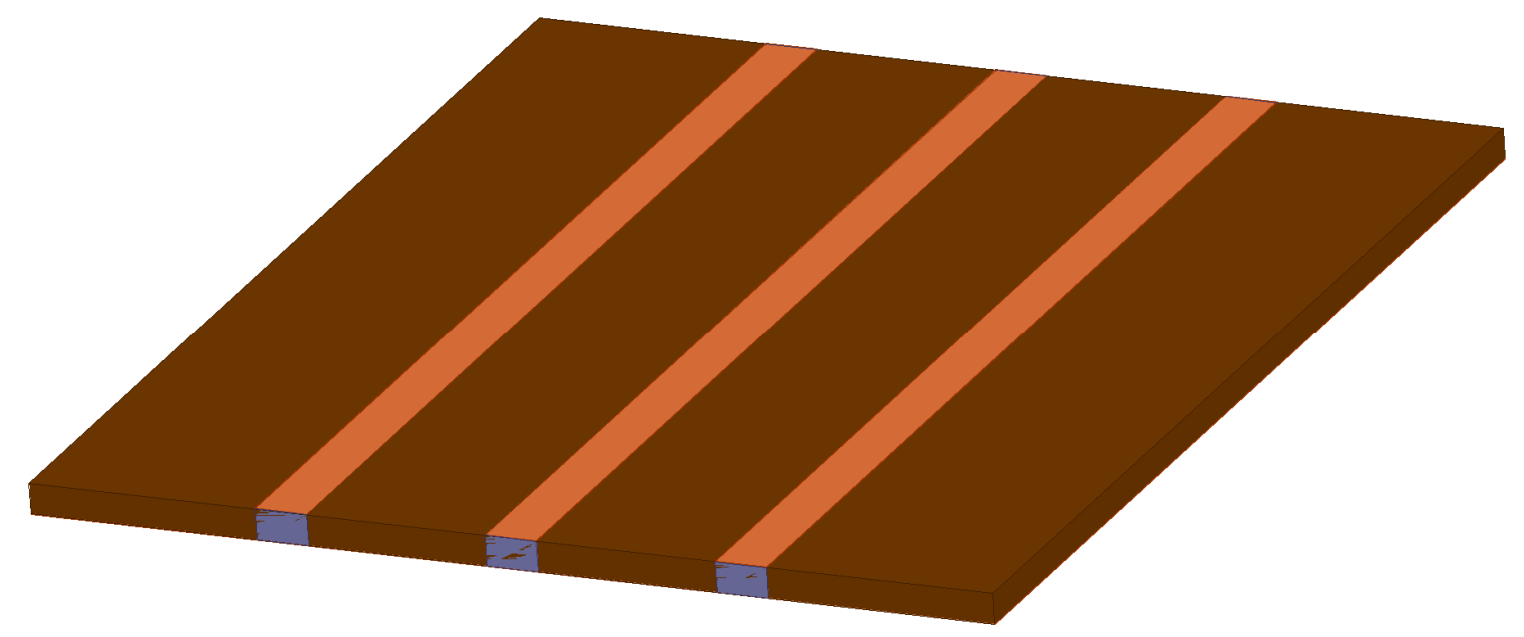

Figure 29. 3D Model of unshielded microstrip in HFSS

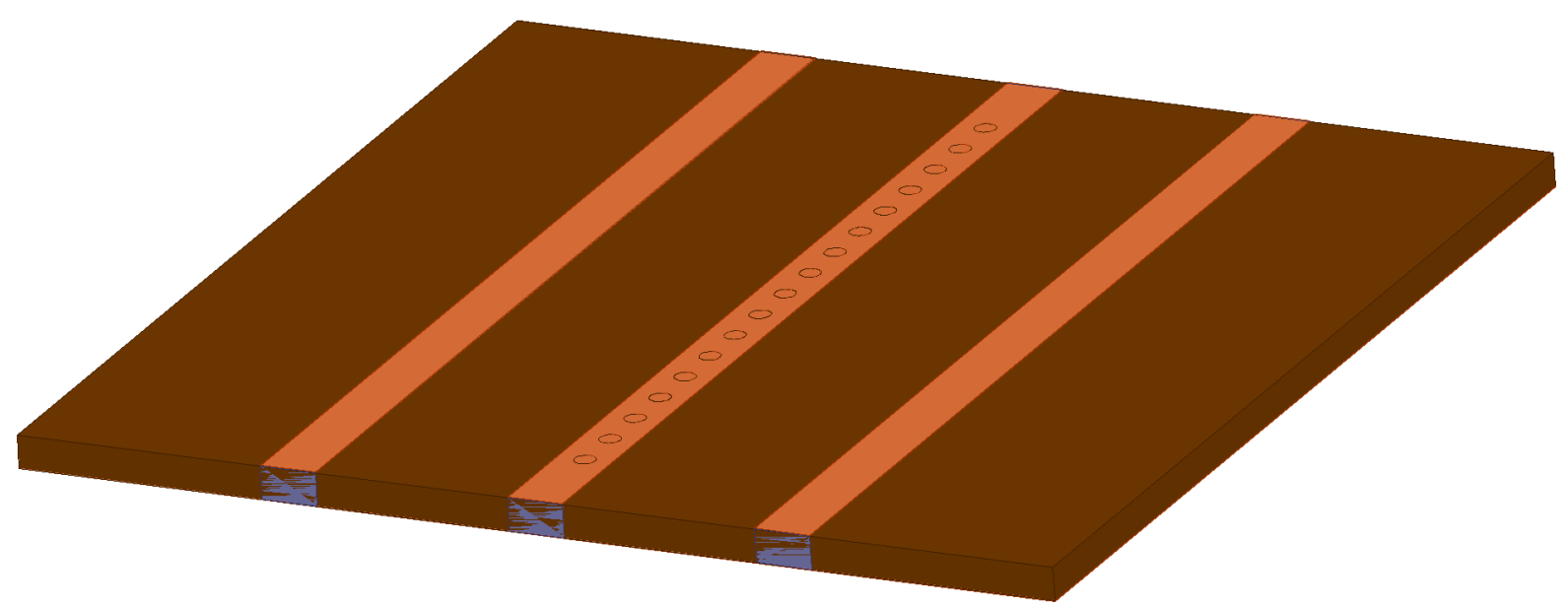

Figure 30. 3D Model of shielded microstrip in HFSS 


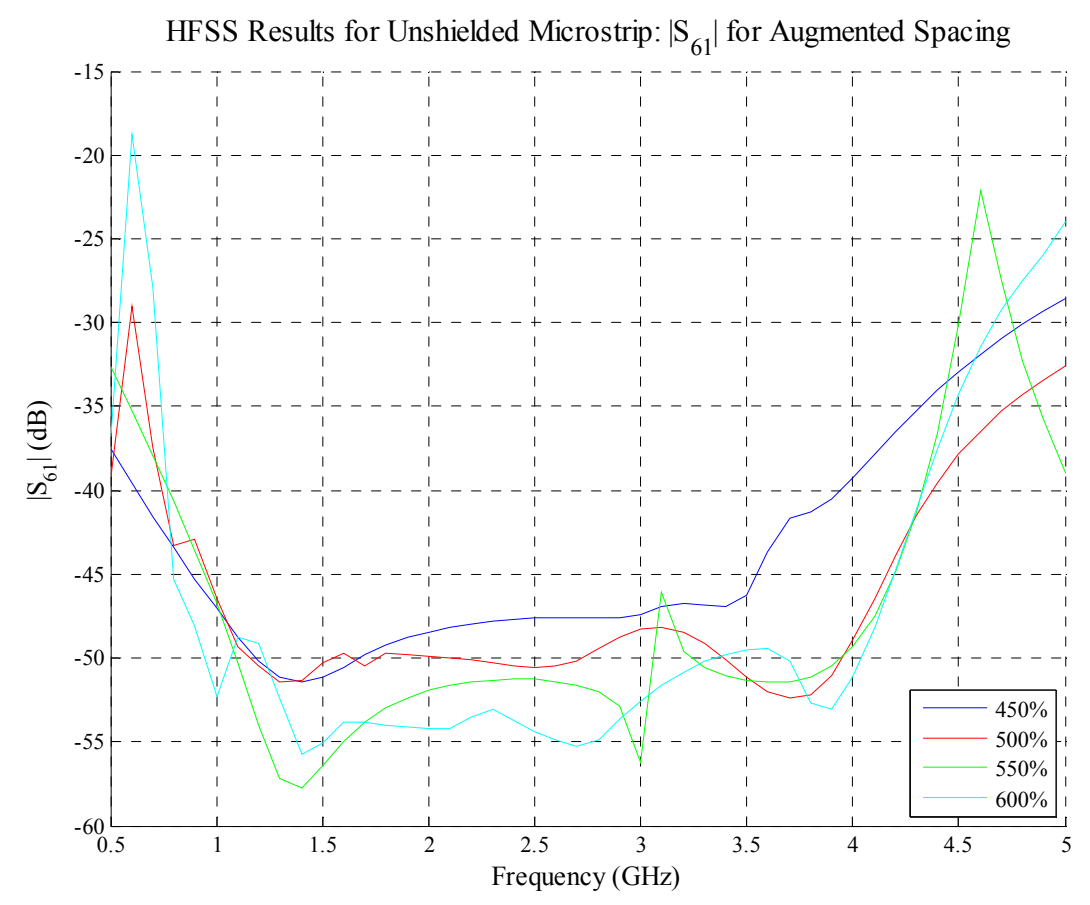

Figure 31. HFSS simulation result for unshielded microstrip vs. frequency

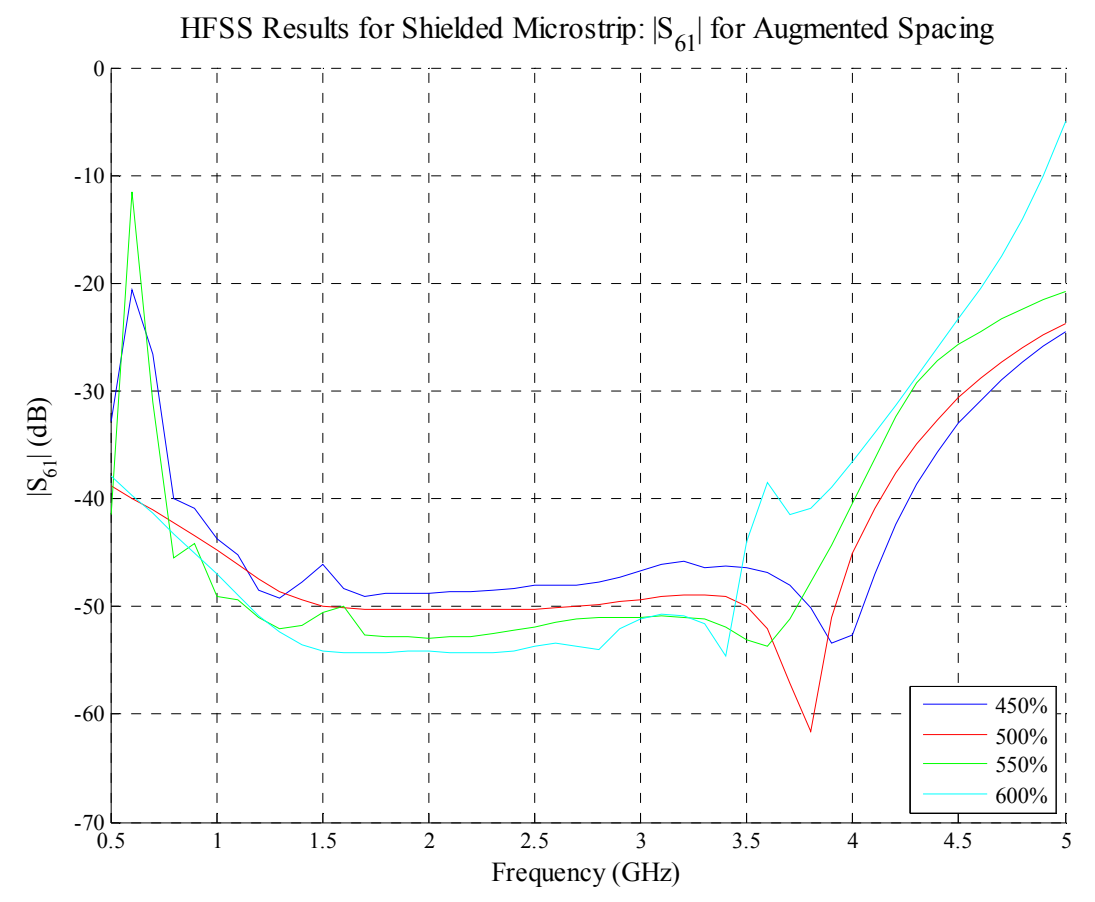

Figure 32. HFSS simulation result for shielded microstrip vs. frequency 


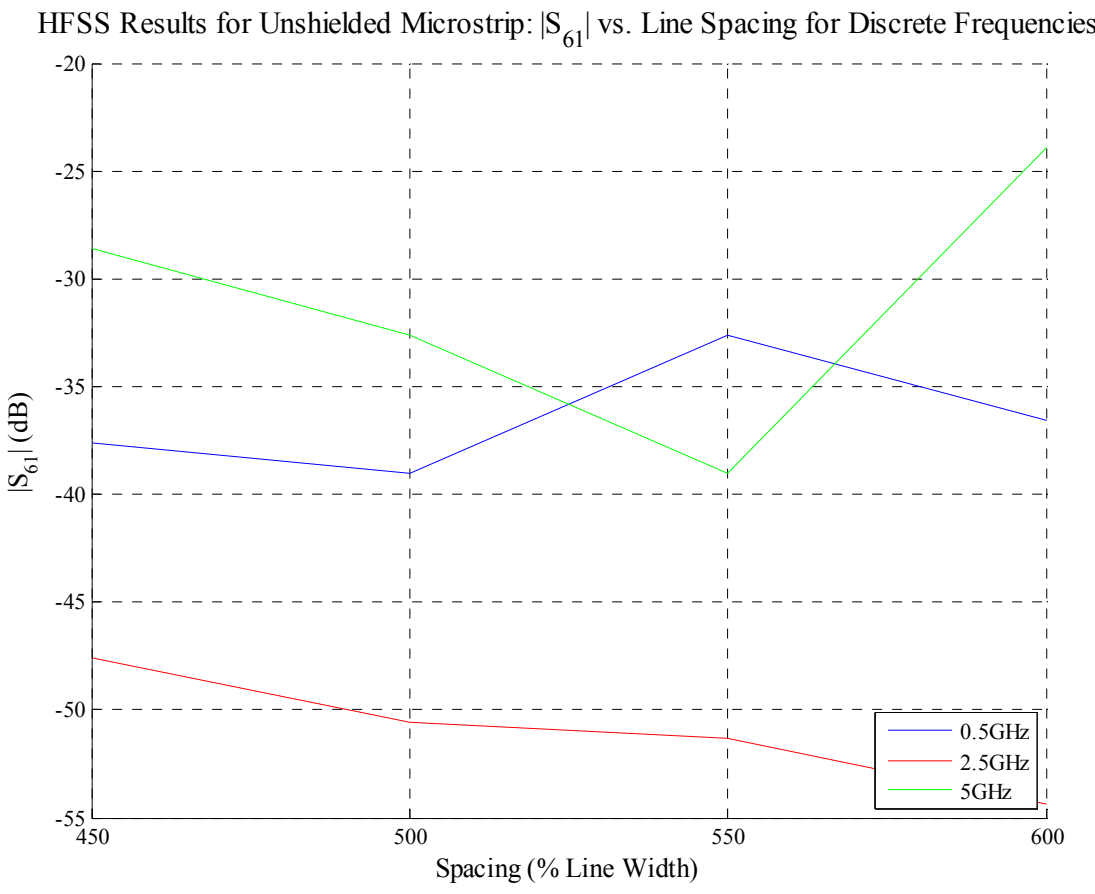

Figure 33. HFSS simulation result for unshielded microstrip vs. line spacing

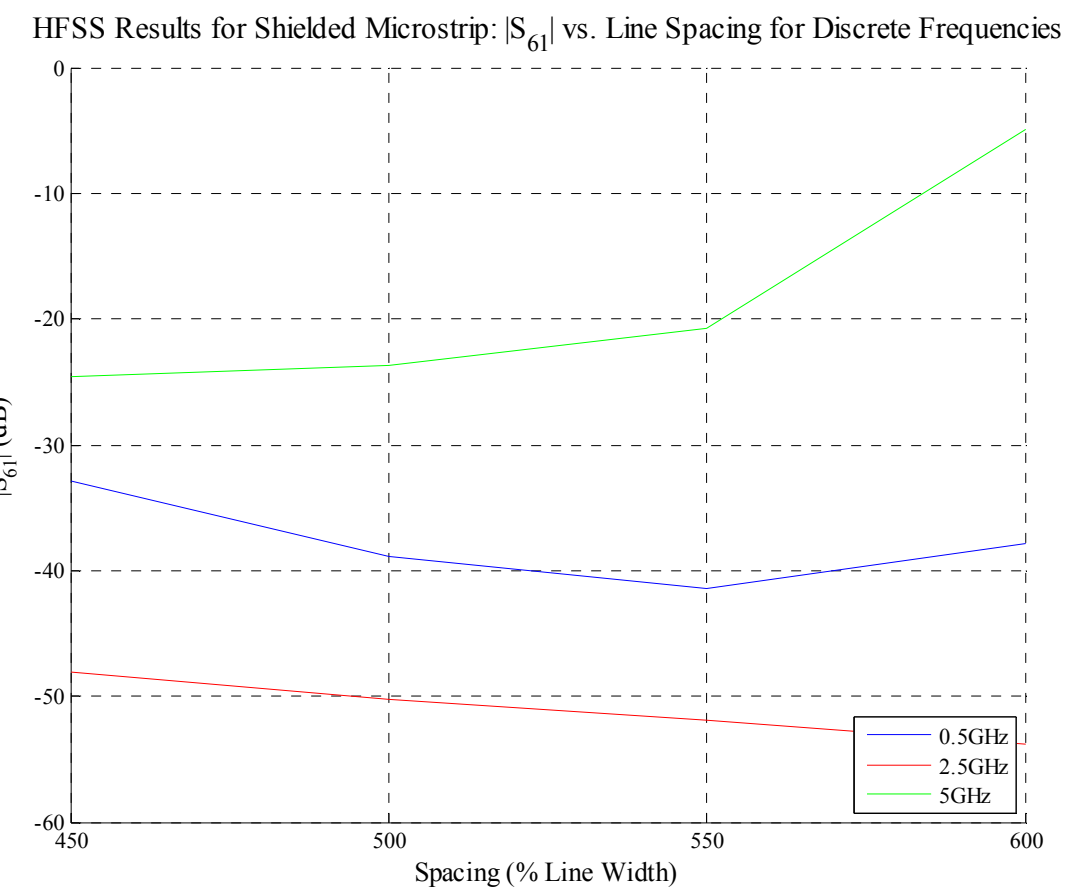

Figure 34. HFSS simulation result for shielded microstrip vs. line spacing 
HFSS Results for Isolation Reduction vs. Frequency for Augmented Spacing

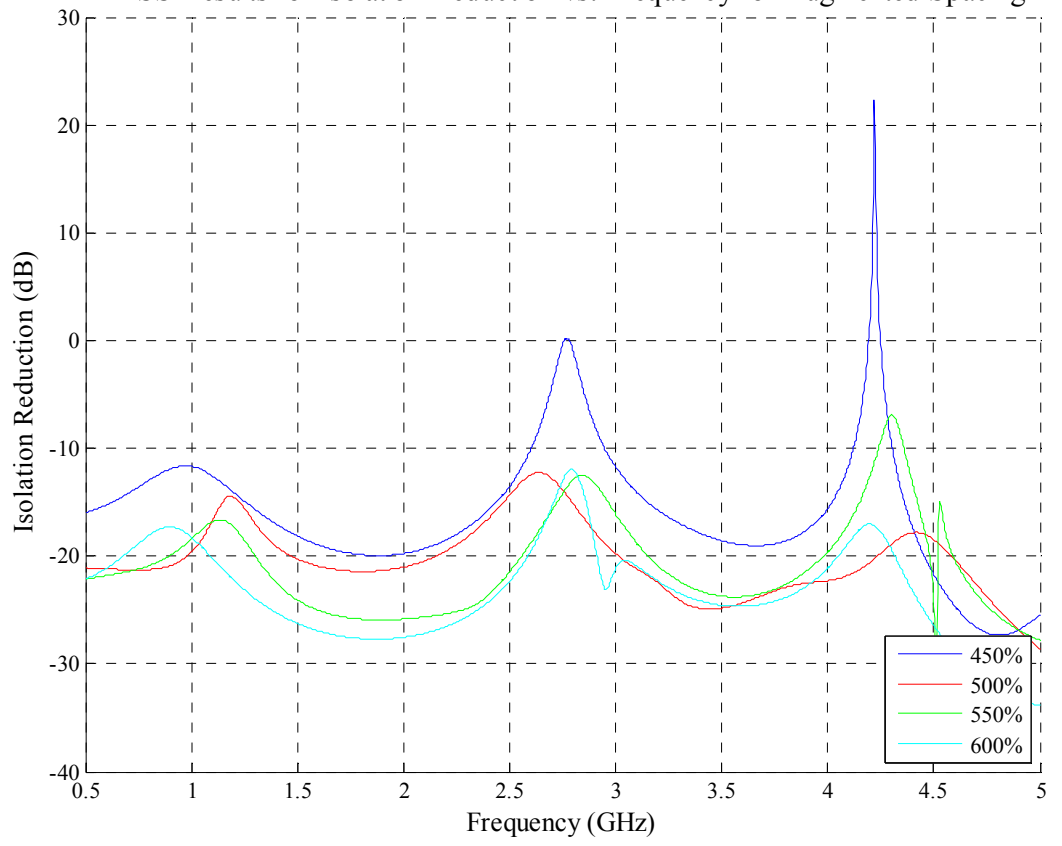

Figure 35. HFSS result for isolation reduction vs. frequency

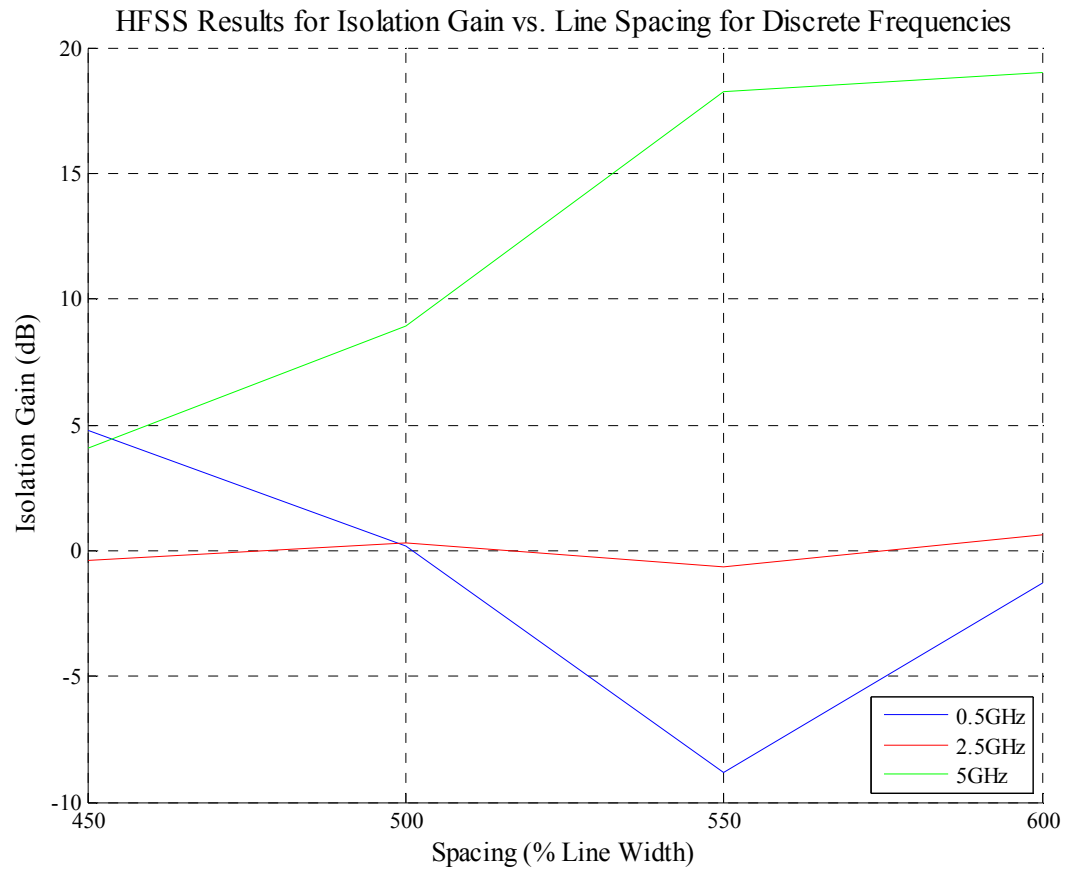

Figure 36. HFSS result for isolation reduction vs. line spacing 
Figures $31 \& 32$ illustrate the magnitude of the crosstalk present in the system for various values of line spacing. According to this data there are certain frequency ranges where a tighter spacing reduces crosstalk for certain frequency bands. Figures $33 \& 34$ present the crosstalk versus line spacing for three different frequencies. Viewing crosstalk in this manner would be beneficial for a designer working on a narrowband system that requires high signal quality. This view also serves to show how cross corner coupling may be greater for higher frequencies, which is counter intuitive but still occurs. Isolation reduction versus frequency and spacing are presented in figures $35 \& 36$. Each plot can illustrate whether or not implementing a shield would be beneficial or not. Values of isolation gain less than 0 clearly indicate that you would only be degrading the structures noise immunity and the values greater than zero would of course enhance the noise immunity.

\subsection{LABORATORY TESTING}

The second component toward the validation of hypothesis put forth in the previous chapters is laboratory testing. Fabrication and testing procedures are covered with vast detail in this section. Microstrip is a planar transmission line that lends itself very well to rapid fabrication methods such as photolithography and circuit board milling machines. Therefore, as established earlier, it will be the transmission line structure that is to be fabricated for experimental validation purposes. As in the computer simulations, FR-4 epoxy will be the only substrate used in fabrication. $50 \Omega$ characteristic impedance on a $0.0625 "(1.5875 \mathrm{~mm})$ substrate gives a line width of $0.106 "(2.6924 \mathrm{~mm})$.

\subsubsection{FABrication Process}

The road to fabrication of coupled microstrip lines begins with a survey of the test equipment available and, not to mention, necessary. The network analyzer available for measurements has male SMA connectors. Therefore, we elected to purchase the narrowest SMA end launch female connector available. The narrowest such connector on the market was $0.375 "(9.525 \mathrm{~mm})$ which imposes a minimum spacing of 0.440 " $(11.176 \mathrm{~mm})$ or $415 \%$ line width between conductors, after taking the land pattern into consideration for $50 \Omega$ microstrip on 0.0625 " $(1.5875 \mathrm{~mm})$ thick FR-4. This minimum spacing was beyond the bounds of the computer simulations already carried out. However, I have hypothesized that the magnitude of $\mathrm{S}_{16}$ for a shielded line will still be about $5 \mathrm{~dB}$ lower than the unshielded line even at values of spacing greater than 
$100 \%$ based on the following simulation result. From the plot below, at $5 \mathrm{GHz}$, the unshielded line has a corner to corner voltage transfer magnitude of $-23.8540 \mathrm{~dB}$ and the shielded line has a value of $-29.1543 \mathrm{~dB}$ giving a difference of $5.300209 \mathrm{~dB}$. So from $10 \%$ spacing to $100 \%$ for $50 \mathrm{Ohm}$ lines fabricated on $1.5875 \mathrm{~mm}$ FR-4 the difference between voltage transfer at $5 \mathrm{GHz}$ remains just about constant, interesting behavior if valid.

The spacing values required for a fabricated board requires the simulation of these structures which is done in HFSS. Installation of end launch connectors requires the insertion of several copper landing pads that straddle the strip the connector will connect to in order to achieve the best possible match for the coaxial to microstrip transition. This is important to the validation because without a proper match between connector and line there will be reflected waves that will distort the results.

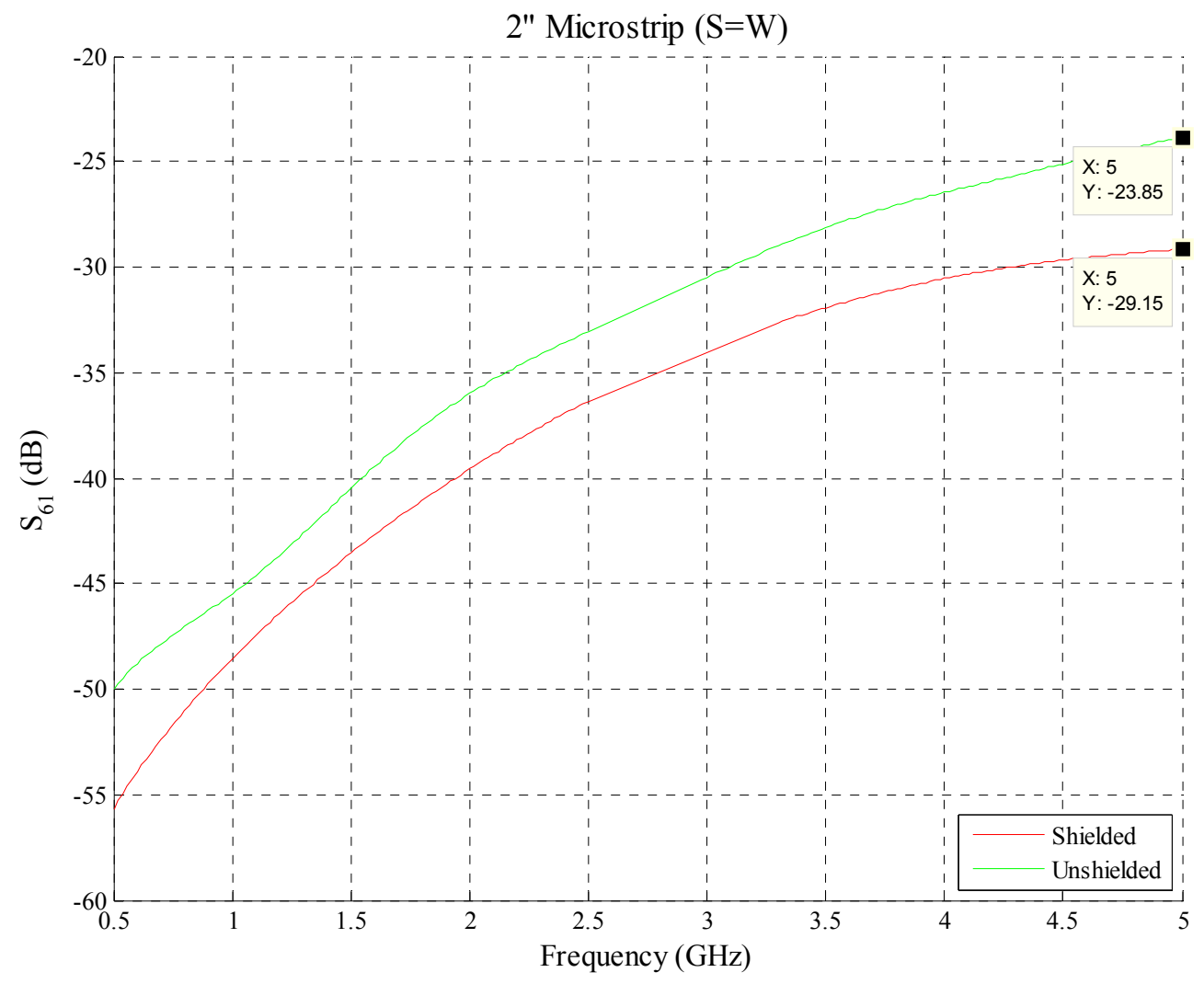

Figure 37. Crosstalk performance comparison of $50 \Omega$ microstrip structures 
Next, the platform that will be used to fabricate the test structures is the LPKF ProtoMat S52. This rapid prototyping printed circuit board milling machine is a good choice because it is time efficient as well as having an auxiliary end mill set with a minimum mill size of 10 mils (254 microns). The software that runs the milling machine, CircuitCAM, accepts many file formats including Gerber and *.dxf. I chose to draw my layout in AutoCAD where I can have a high degree of control of line spacing and implement the connector land patterns with extreme ease. I was able to create a $1 \times 4$ array of boards after the completion of the first and then adjust the spacing from $450 \%$ to $500 \%$ for the second board and so forth. Then create a $2 \times 1$ array of those four boards and place 17 equally spaced through holes down the length of the center conductor.

From this point, the file is saved as an AutoCAD 2000/LT2000 DXF so that I have full compatibility with CircuitCAM. In the CircuitCAM environment the details for board outline and copper rubout are specified.

With the CircuitCAM file complete, fabrication begins. The file exported from CircuitCAM is then imported into Board Master which is the software that controls the LPKF ProtoMat S52. The tools necessary for the fabrication were defined in CircuitCAM which are then loaded into the ProtoMat along with the double sided half ounce FR-4 board. The milling process is initiated, followed by the drilling, followed by the contour routing which allows the boards to be extracted from the source material.

Once the boards are extracted from the source material, connectors can now be mounted onto them. First, the through holes must be shorted to the ground plane by requirement for the SMA connectors and the center conductors for the experiment. The shielded boards require short circuits to be placed in through holes placed down the center conductor. This is accomplished by taking 30AWG enamel coated copper wire and threading it in a zig-zag pattern down the length of the board trying to minimize the air gap between wire and copper strip. The areas to be soldered are treated with rosin flux to allow for easy soldering without having to apply excessive heat. An example of a final build is shown in figure 42. Four of the six connectors have $50 \Omega$ terminations in place. This image shows how a board would be connected to a two port vector network analyzer to characterize corner to corner coupling $\left(\mathrm{S}_{61}\right)$. 

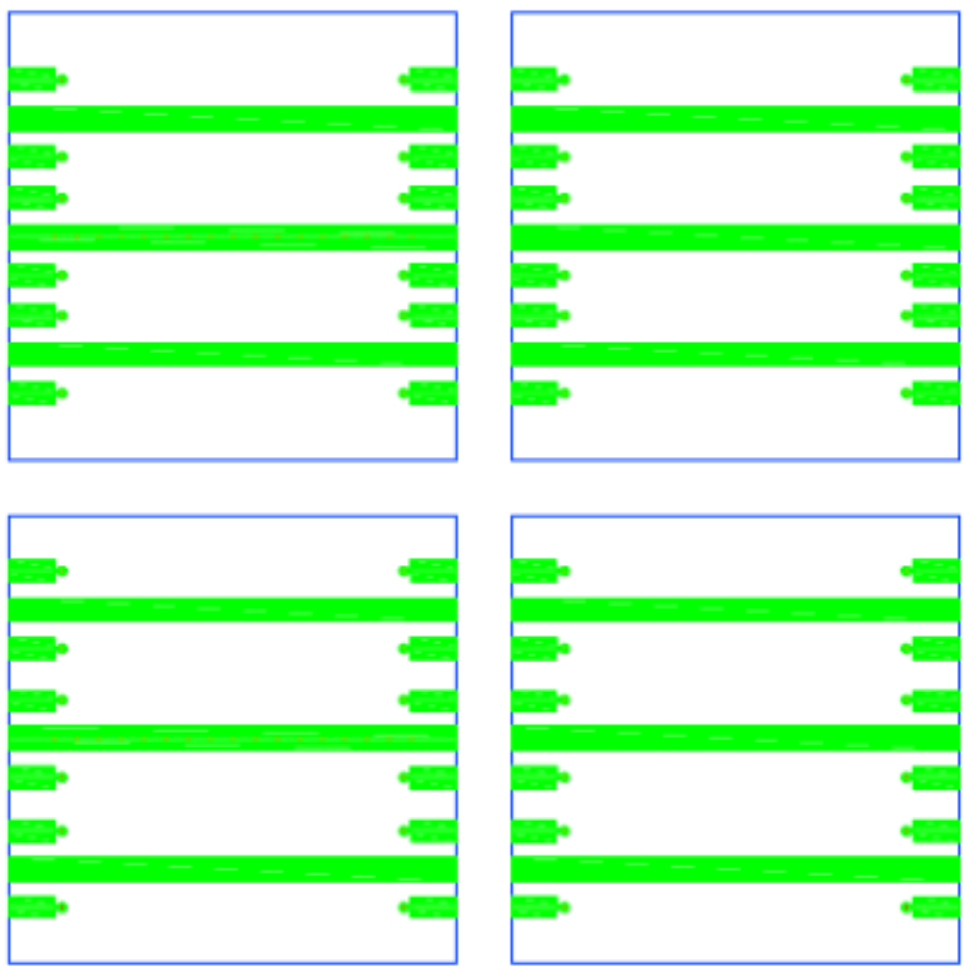

Figure 38. AutoCAD drawing used for board fabrication 


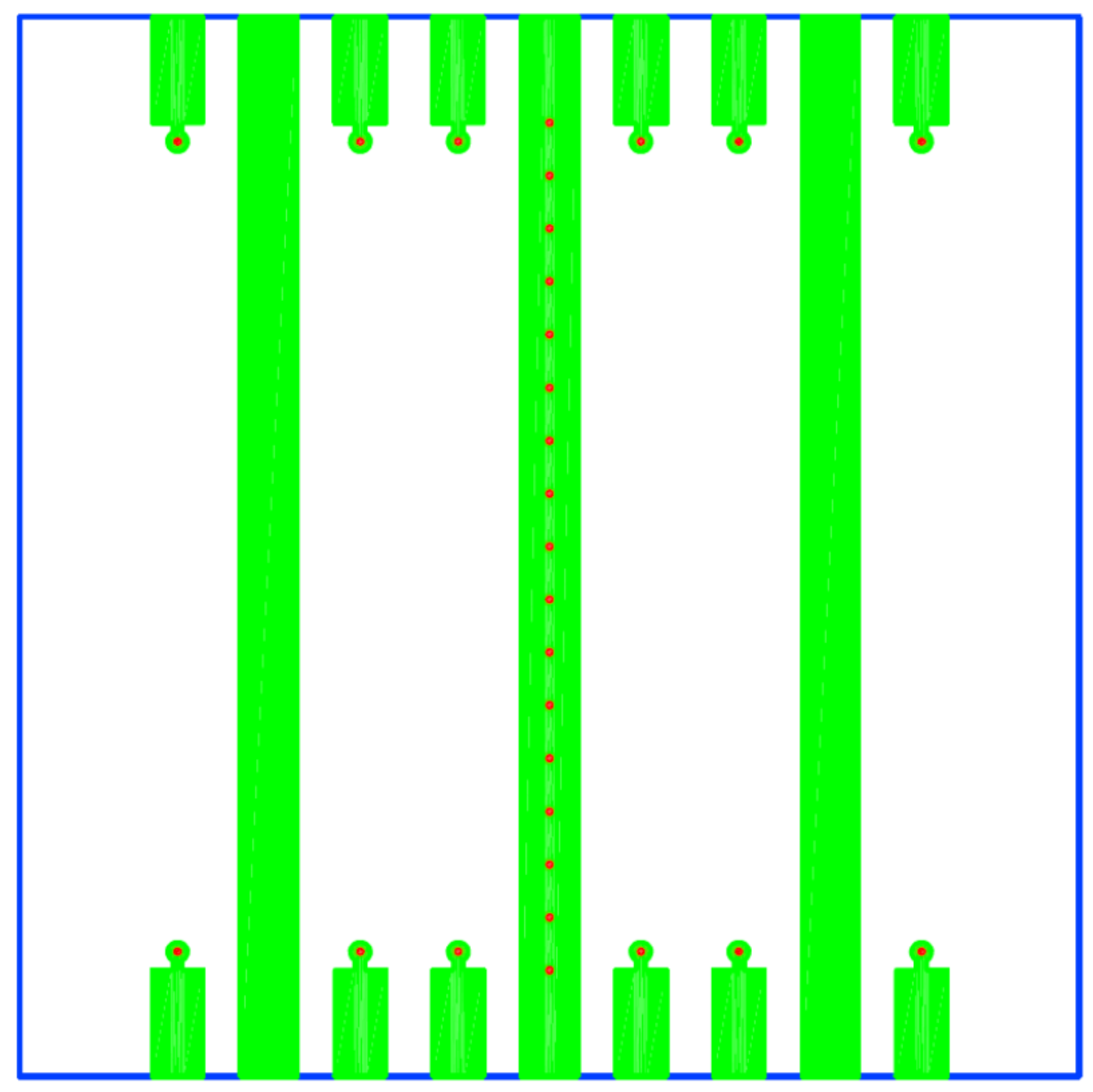

Figure 39. Detailed view of a shielded microstrip structure to be fabricated 


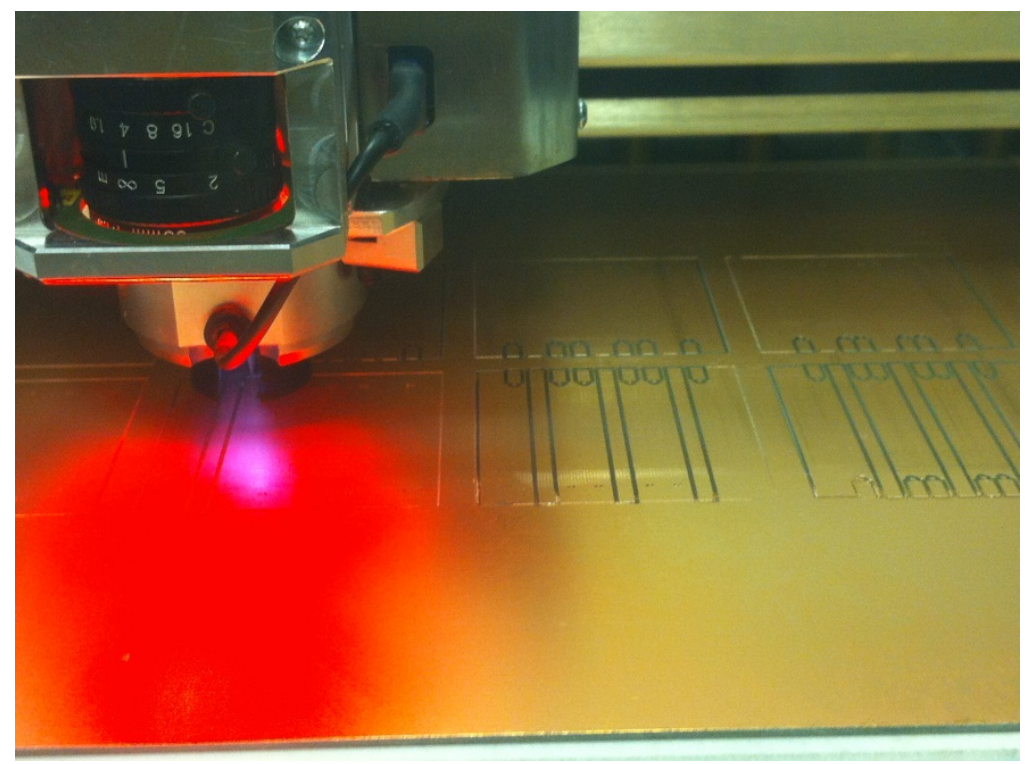

Figure 40. LPKF Protomat S52 milling a set of boards

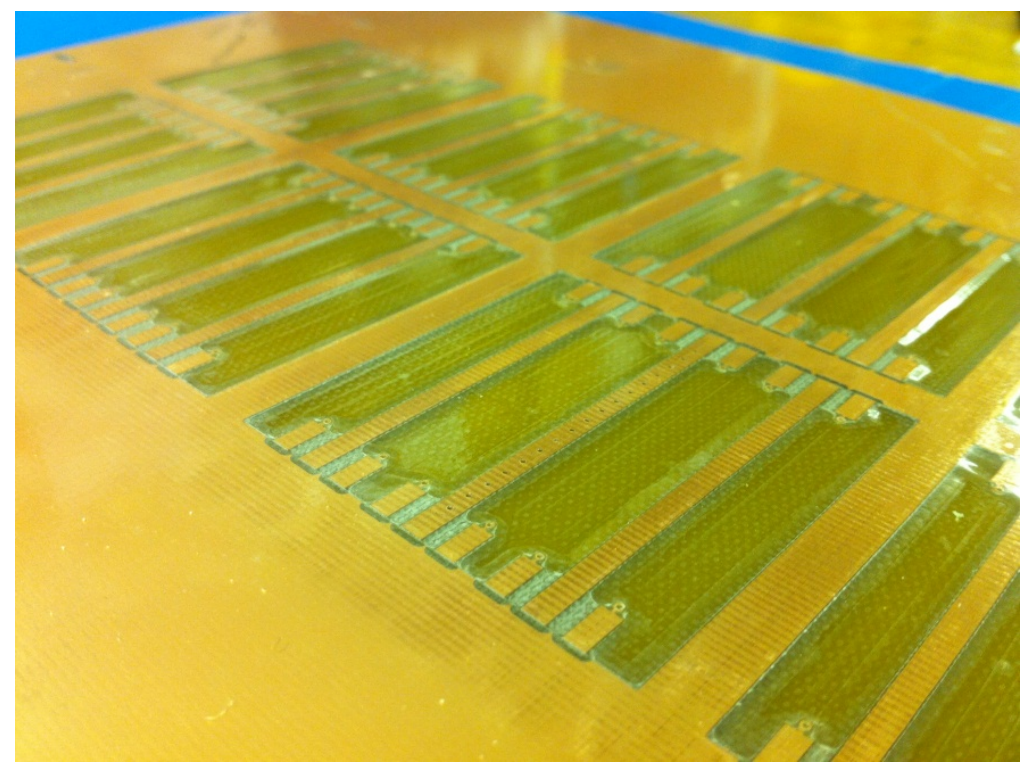

Figure 41. A set of milled boards before contour routing phase 


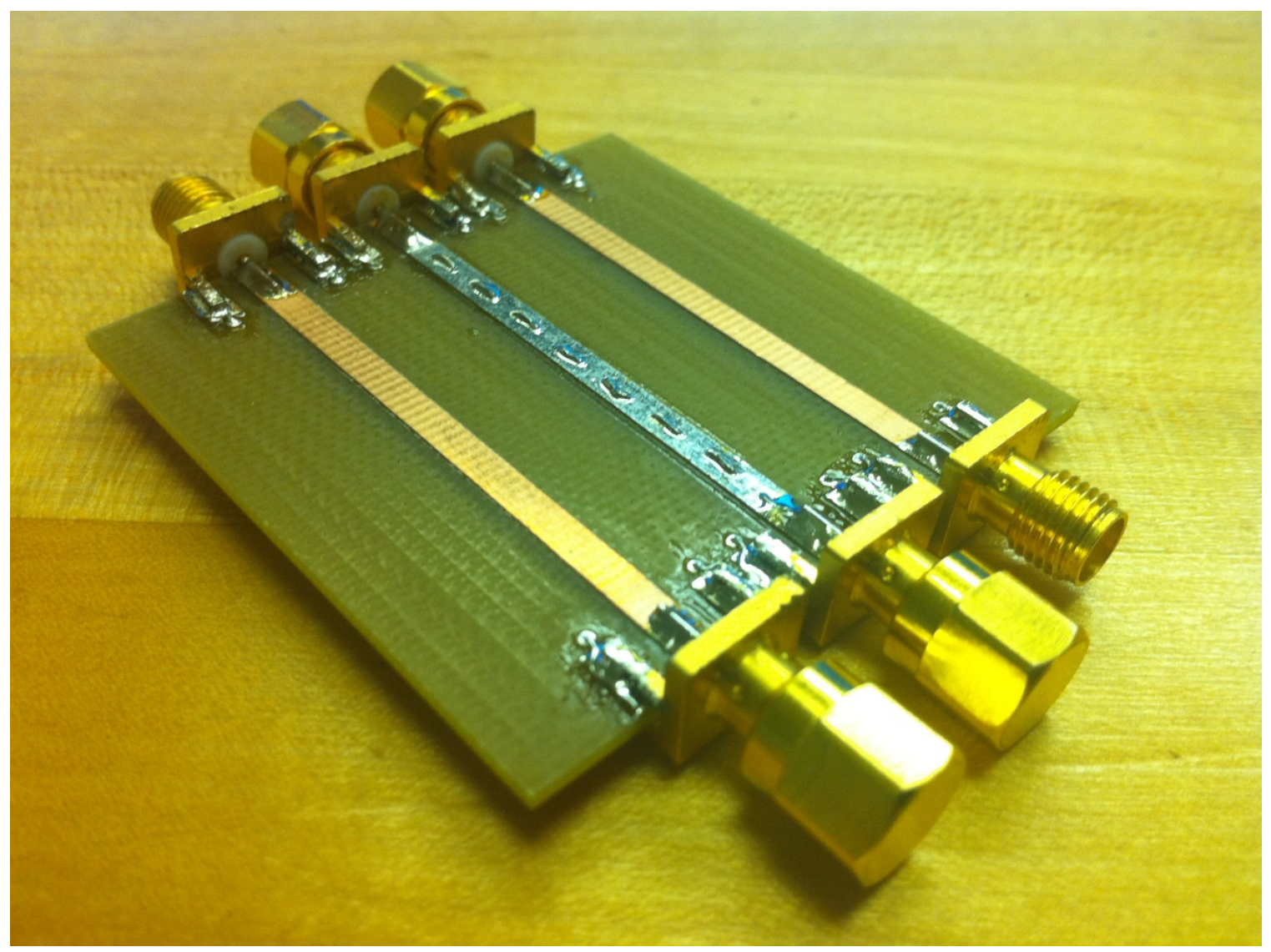

Figure 42. Completed shielded structure ready for crosstalk measurement $\left|S_{61}\right|$

\subsubsection{Measurement Considerations}

Measurements were taken on an Agilent 8720ES Vector Network Analyzer. The symmetry of the structure allows for symmetry to lower the number of measurements taken illustrated below.

$$
S=\left[\begin{array}{llllll}
S_{11} & S_{12} & S_{13} & S_{14} & S_{15} & S_{16} \\
S_{21} & S_{22} & S_{23} & S_{24} & S_{25} & S_{26} \\
S_{31} & S_{32} & S_{33} & S_{34} & S_{35} & S_{36} \\
S_{41} & S_{42} & S_{43} & S_{44} & S_{45} & S_{46} \\
S_{51} & S_{52} & S_{53} & S_{54} & S_{55} & S_{56} \\
S_{61} & S_{62} & S_{63} & S_{64} & S_{65} & S_{66}
\end{array}\right]
$$




\begin{tabular}{|c|c|}
\hline \multicolumn{2}{|c|}{ Symmetry Breakdown } \\
\hline Reflection in Side Lines & $\mathrm{S}_{11}=\mathrm{S}_{22}=\mathrm{S}_{55}=\mathrm{S}_{66}$ \\
\hline Reflection in Center Line & $\mathrm{S}_{33}=\mathrm{S}_{44}$ \\
\hline Throughput Side Lines & $\mathrm{S}_{12}=\mathrm{S}_{21}=\mathrm{S}_{56}=\mathrm{S}_{65}$ \\
\hline Throughput Center Line & $\mathrm{S}_{34}=\mathrm{S}_{43}$ \\
\hline Next Line Adjacent Side Induction & $\mathrm{S}_{13}=\mathrm{S}_{31}=\mathrm{S}_{24}=\mathrm{S}_{42}=\mathrm{S}_{35}=\mathrm{S}_{53}=\mathrm{S}_{46}=\mathrm{S}_{64}$ \\
\hline Next Line Opposite Corner & $\mathrm{S}_{14}=\mathrm{S}_{41}=\mathrm{S}_{23}=\mathrm{S}_{32}=\mathrm{S}_{36}=\mathrm{S}_{63}=\mathrm{S}_{45}=\mathrm{S}_{54}$ \\
\hline Side Corners & $\mathrm{S}_{15}=\mathrm{S}_{51}=\mathrm{S}_{26}=\mathrm{S}_{62}$ \\
\hline Cross Corners & $\mathrm{S}_{16}=\mathrm{S}_{61}=\mathrm{S}_{25}=\mathrm{S}_{52}$ \\
\hline
\end{tabular}

Table 2. Types of symmetry present in scattering parameter matrix used to reduce number of measurements needed

There were 3 types of structures fabricated for testing: Dual Line, Unshielded Line and Shielded Line. The only structure of the three that can take full advantage of the symmetry and have the added advantage of higher data transmission rate because of having a third transmitting (or receiving) line is the unshielded structure. Dual and shielded line structures may or may not have the advantage of higher signal integrity, that is what is being validated.

Taking these symmetries into consideration it is easy to see that the only port combinations that must be measured for an unshielded geometry are $1 \& 2,1 \& 3,1 \& 4,1 \& 5,1 \& 6$ and $3 \& 4$ in order to determine the entire scattering matrix. Dual and shielded lines will be measured at the same ports with the exception of $1 \& 3,1 \& 4$ and $3 \& 4$. Shielded line measurements were taken under 4 different conditions that will be presented in the following sections. 


\section{ChAPTER 5}

\section{Measurement Results}

All measurements were exported to a text(*.csv) file format along with an image $(*$.jpg) file which are included in Appendix B. All text based data was imported into the MATLAB environment for postprocessing and comparison between structures.

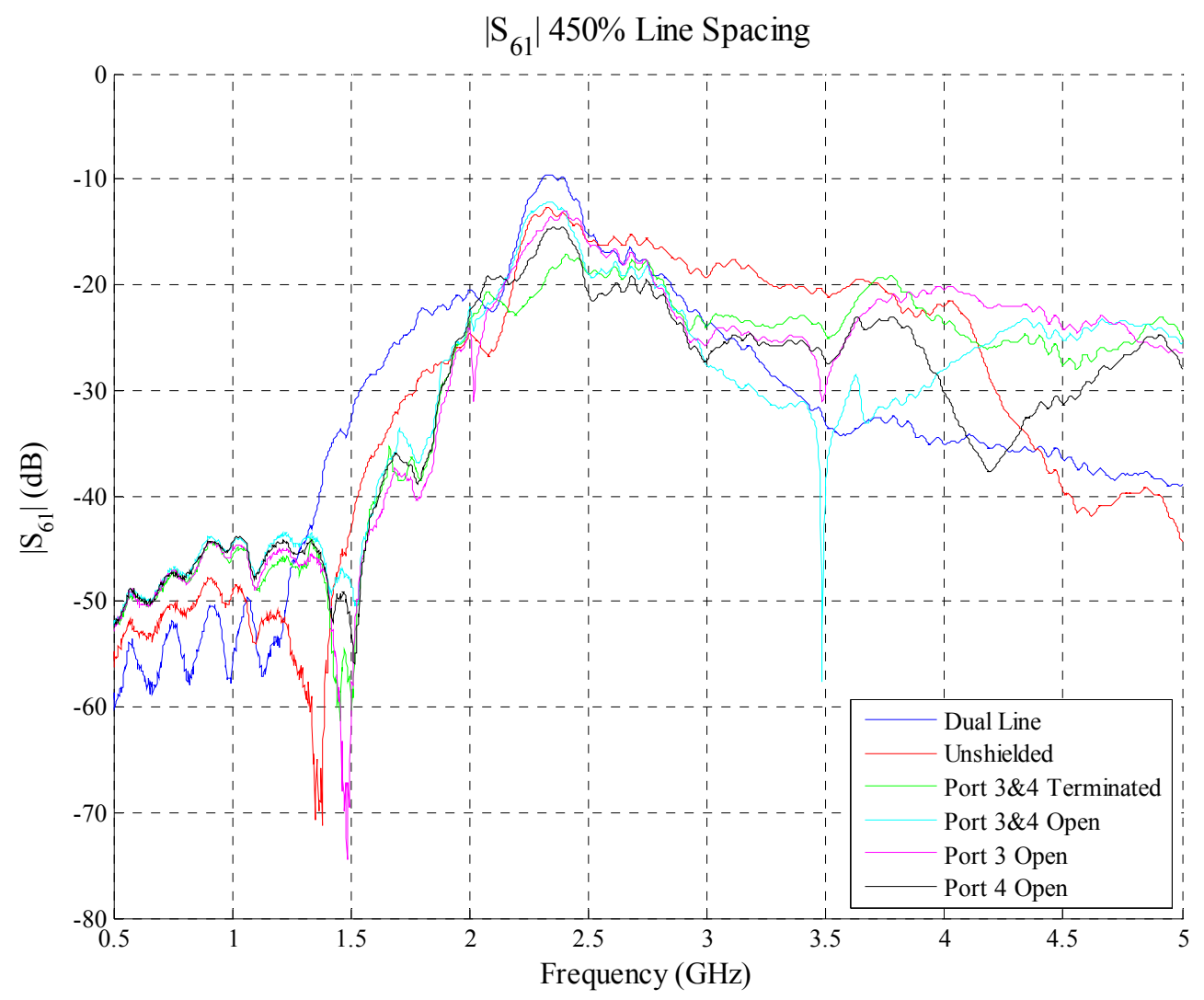

Figure 43. Measured crosstalk of all $450 \%$ spaced structures

The figure above shows a family of curves for $\mathrm{S}_{61}$ for the different structures. Recall that $\mathrm{S}_{61}$ is the cross corner voltage transfer. The curves illustrate that from $500 \mathrm{MHz}$ to about $1.3 \mathrm{GHz}$ that the guarded lines have no effect in comparison to the unshielded three line structure. The dual line is the structure that performs the best in this band. At about $1.4 \mathrm{GHz}$ there is a transition to where shielded lines will have lower values for $\mathrm{S}_{61}$. When considering only the shielded lines and the unshielded lines, the shielded lines effectively lower the coupling between opposite corners from $1.4 \mathrm{GHz}$ up to about $4.25 \mathrm{GHz}$. After that 
point the shielding mechanism loses its effectiveness. The following figure shows the side line throughput which could help give a clue as to how much energy is being radiated from the side line over to the adjacent lines.

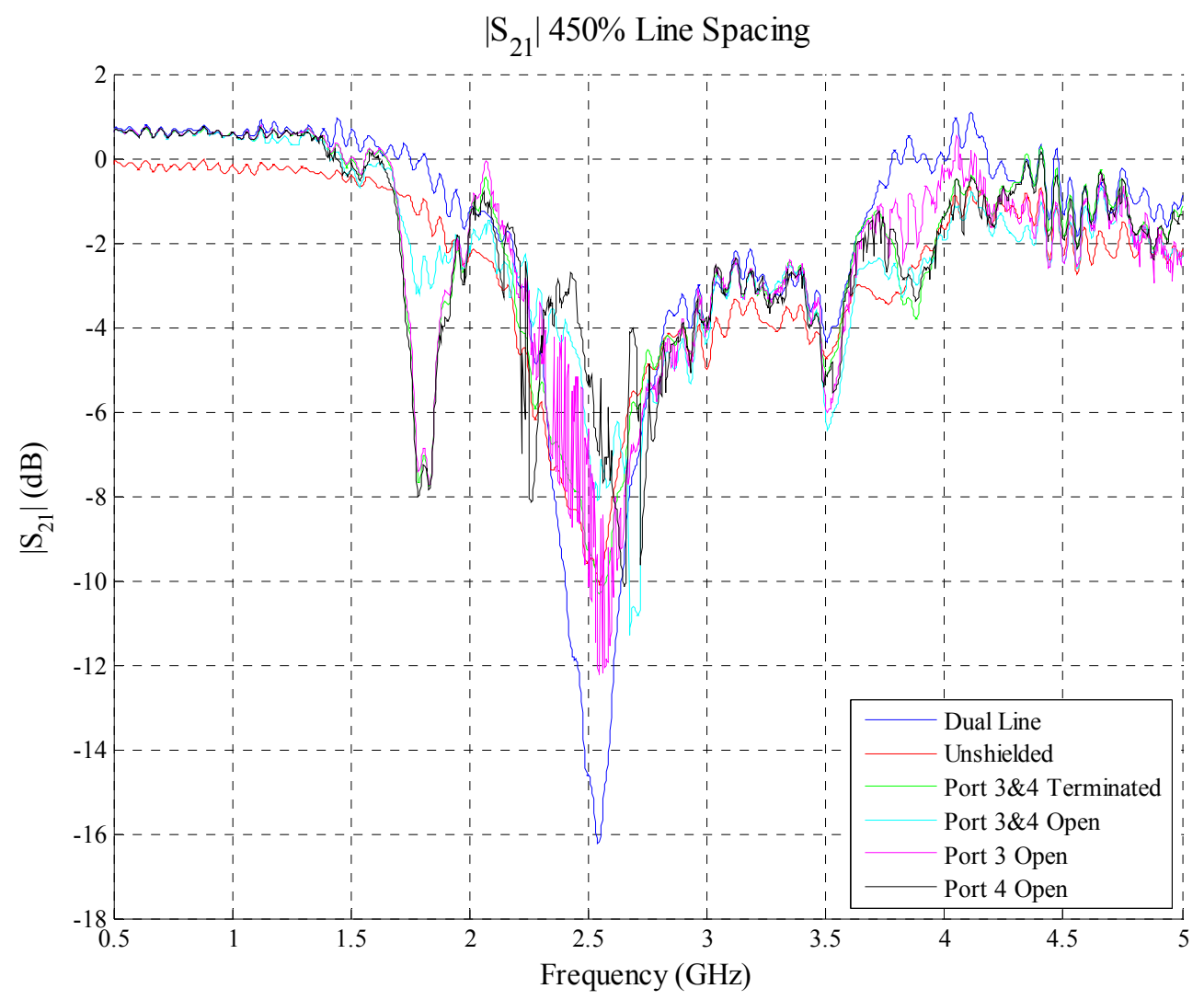

Figure 44. Measured throughput for all 450\% spaced structures

From this family of curves there is an immediate discrepancy. The received wave at port 2 has greater amplitude than the incident wave at port 1 and this is a completely passive structure so this is either a calibration error of the instrument or an impedance mismatch. There are various losses of signal throughput due to line resonances.

A graphic of $\mathrm{S}_{11}$, better known as the reflection coefficient in this case, show the points at which resonance occurs for different structures. The marker in the figure for $\mathrm{S}_{11}$ shows a resonant peak where $2.23 \mathrm{GHz}$ is the 
resonant frequency with a reflection coefficient value of $-38.34 \mathrm{~dB}$ or 0.0121 . Input impedance at this frequency is defined in terms of the reflection coefficient, $\Gamma$, by the following expression.

$$
Z_{I N}=Z_{0} \frac{1+\Gamma}{1-\Gamma}=(50 \Omega) \frac{1-0.0121}{1+0.0121}=51.23 \Omega
$$

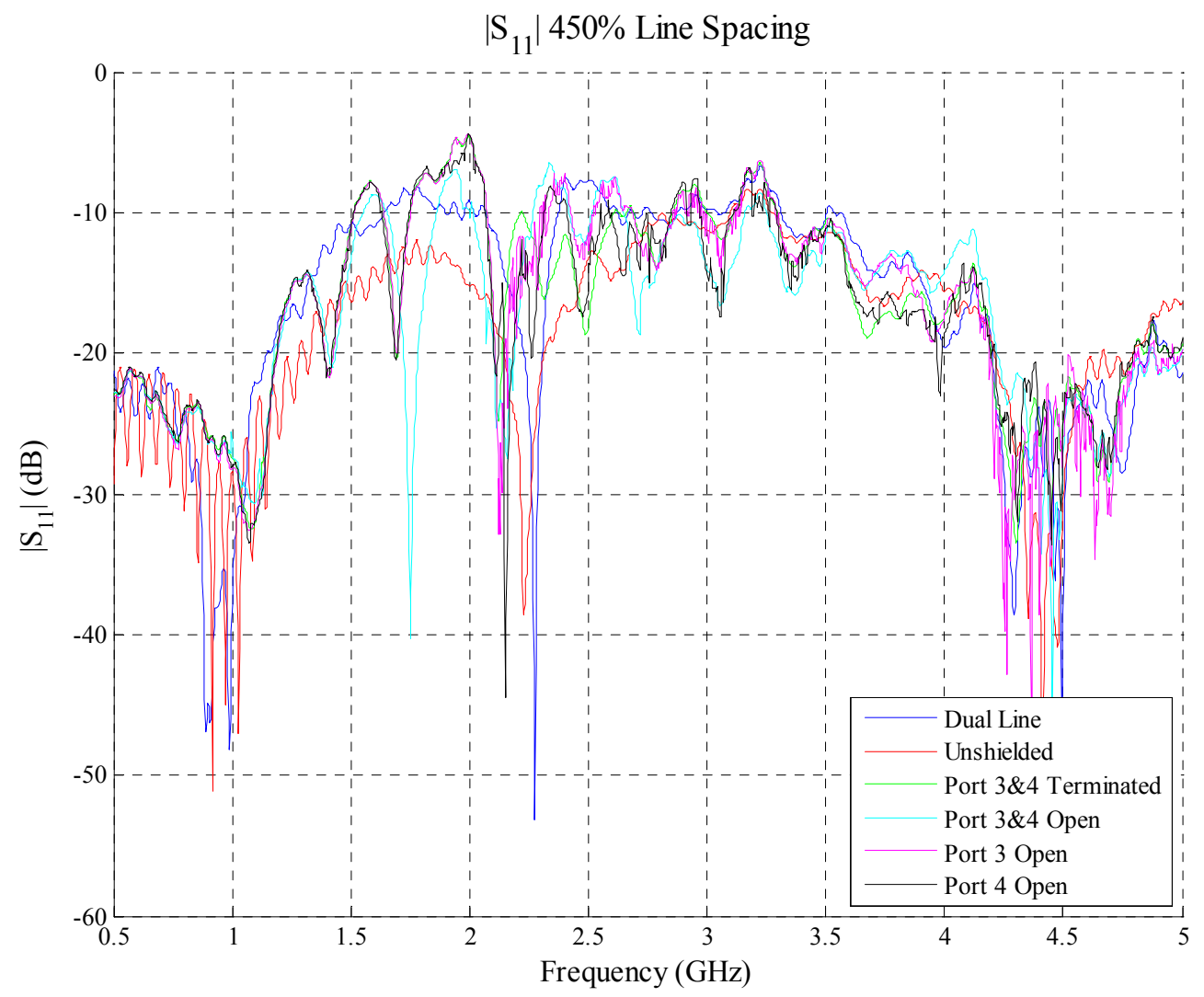

Figure 45. Input reflection coefficient for all 450\% spaced structures

The effective dielectric constant for this structure can be calculated by the following expression:

$$
\nu_{p}=\frac{c}{\sqrt{\varepsilon_{e}}}=f_{0} \lambda
$$

It is apparent that the half wavelength is at this frequency because it is numerically close to the free space wavelength. Where wavelength is two times the line length $(\lambda=21)$. Substituting this for $\lambda$, we obtain. 


$$
\varepsilon_{e}=\left(\frac{c}{2 f_{0} l}\right)^{2}=1.7533
$$

This result is as expected given that the effective dielectric constant should lie between unity and the relative dielectric constant of the substrate, which for FR-4 fiberglass epoxy is $\approx 4.9$.

\section{Scattering Parameters for Three Line Structure at 450\% Line Spacing}
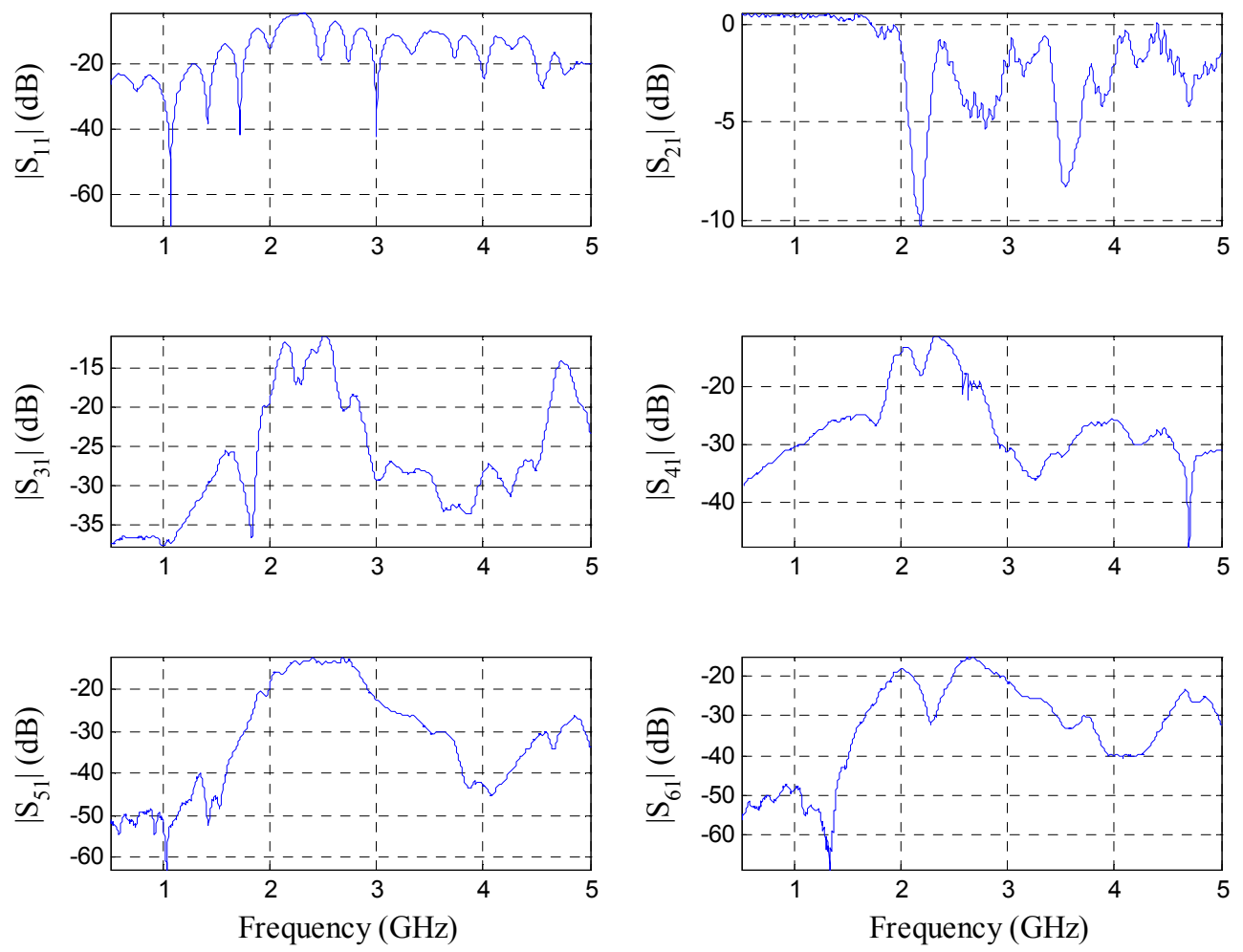

Figure 46. Scattering matrix magnitude for 450\% Line Spacing

The sum of the magnitude squared of elements in the first column of the scattering matrix is theoretically equal to unity for a lossless network where the structure does not radiate or suffer losses to finite conductivity and the dielectric's small but finite conductivity due to displacement current. Theoretically because the system is isolated from other sources of radiation and time varying fields in simulation. For the unshielded structure at $450 \%$ spacing the sum of each element in the first column dotted with its complex conjugate is calculated to get a numerical grasp as to how much energy has entered or been dissipated. 


$$
\begin{gathered}
\sum_{n=1}^{6} S_{n 1} S_{n 1}^{*}=S_{11} S_{11}^{*}+S_{21} S_{21}^{*}+S_{31} S_{31}^{*}+S_{41} S_{41}^{*}+S_{51} S_{51}^{*}+S_{61} S_{61}^{*} \\
=\left|S_{11}\right|^{2}+\left|S_{21}\right|^{2}+\left|S_{31}\right|^{2}+\left|S_{41}\right|^{2}+\left|S_{51}\right|^{2}+\left|S_{61}\right|^{2}
\end{gathered}
$$

Because all ports are terminated into the characteristic impedance of the line, the total power incident at each port will be,

$$
P_{\text {OUT }}=\frac{\left|V_{1}^{+}\right|^{2}}{2 Z_{0}} \sum_{n=1}^{6} S_{n 1} S_{n 1}^{*}
$$

The network analyzer was set to stimulate at a constant $1 \mathrm{~mW}(0 \mathrm{dBm})$ for all frequencies stimulated.

Therefore, the difference between input and output power will reveal how much energy was lost or gained in the system.

$$
\Delta P=P_{I N}-P_{\text {OUT }}=P_{I N}-\frac{\left|V_{1}^{+}\right|^{2}}{2 Z_{0}} \sum_{n=1}^{6} S_{n 1} S_{n 1}^{*}
$$

Positive values of $\Delta \mathrm{P}$ mean that the structure has lost energy to finite conductivity of the conductor and substrate, displacement current in the dielectric and radiation. Negative values of $\Delta \mathrm{P}$ mean that the system has gained energy from external electromagnetic fields. Power loss to the dielectric can be calculated by the following expression.

$$
\alpha_{d}=\frac{k_{0} \varepsilon_{r}\left(\varepsilon_{e}-1\right) \tan \delta}{2 \sqrt{\varepsilon_{e}}\left(\varepsilon_{r}-1\right)}(N p / m)
$$

Where $\mathrm{k}_{0}$ is the free-space propagation constant linearly proportional to frequency. The attenuation per unit length due to conductor losses can be found by.

$$
\alpha_{c}=\frac{R_{s}}{Z_{0} W}=\frac{1}{Z_{0} W} \sqrt{\frac{\pi \mu_{0} f}{\sigma_{C u}}}(\mathrm{~Np} / \mathrm{m})
$$

The total attenuation for $50 \Omega$ microstrip line two inches in length $(50.8 \mathrm{~mm})$ can be found by the following. 


$$
P_{\text {LOSS }}=P_{I N}-P_{O U T}=P_{I N}\left(1-e^{-2 l\left(\alpha_{c}+\alpha_{d}\right.}\right)
$$

Input impedance can be found in order to calculate the incident voltage at port one.

$$
\Gamma_{I N}(f)=\frac{Z_{I N}(f)-Z_{0}}{Z_{I N}(f)+Z_{0}} \Rightarrow Z_{I N}(f)=Z_{0} \frac{1+\Gamma_{I N}(f)}{1-\Gamma_{I N}(f)}
$$

The calculation of $\left|\mathrm{V}_{1}^{+}\right|$is as follows,

$$
P_{I N}=\frac{\left|V_{1}^{+}\right|^{2}}{2 \operatorname{Re}\left[Z_{I N}(f)\right]} \Rightarrow\left|V_{1}^{+}\right|=\sqrt{2 P_{I N} \operatorname{Re}\left[Z_{I N}(f)\right]}
$$

In order for input power to remain constant the incident voltage must also vary with frequency which is shown by the frequency dependence of the input impedance.

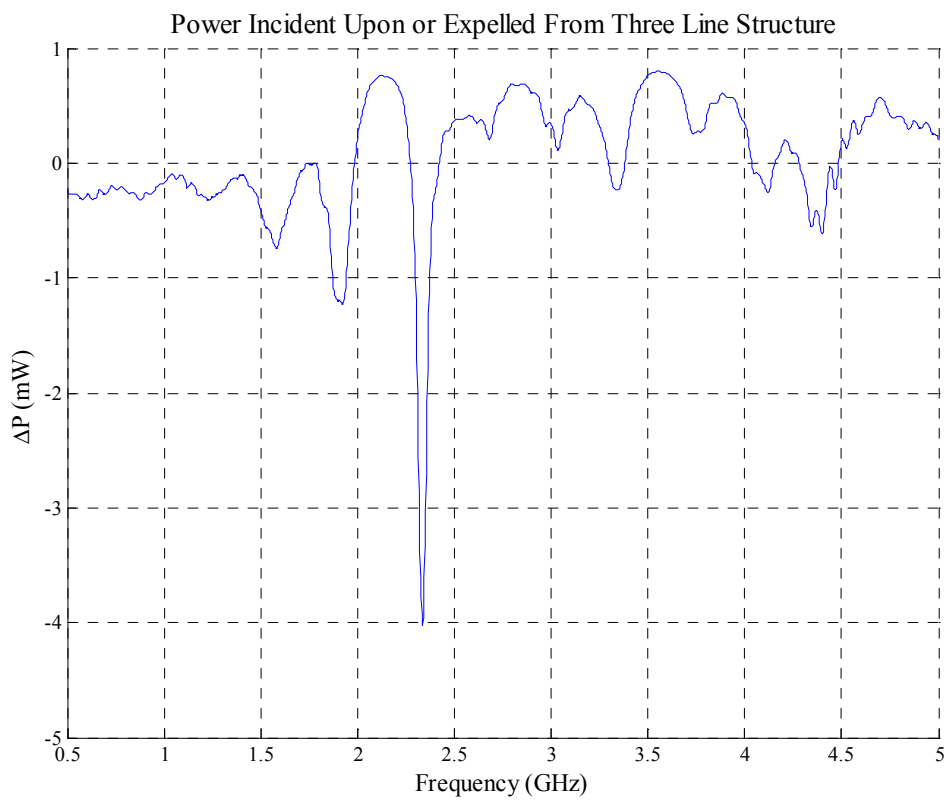

Figure 47. Three line structure power gain/loss

Figure 47 shows that there is a large amount of external interference in the measurements. The greatest amount of incident energy being around 2.4GHz.This result makes sense because there are active cellular 
phones and Bluetooth devices in the laboratory. Loss due to all factors is present at every frequency, but the non-encapsulated geometry of microstrip permits any incident electromagnetic waves to create noise on the structure.

Measured isolation reduction versus frequency with respect to either the dual or three line structure plots are shown in figures 48-55. Each dual/three line pair of plots illustrates isolation reduction for the shielded structure under certain termination conditions. Each of these plots would be useful for a layout engineer because it would allow him/her to see at what frequency band and spacing he/she would be able to apply a shielding technique and achieve that much better noise immunity. For example, if a designer was debating whether or not two transmission lines could be spaced $450 \%$ and saw that the crosstalk magnitude from an extracted scattering parameter was borderline high for his/her application in the 4.5 to $5 \mathrm{GHz}$ band then he/she could refer to figure 48 and see that crosstalk can be reduced by at least $10 \mathrm{~dB}$ for the majority of the band. If the application were in the 1 to $2 \mathrm{GHz}$ band then crosstalk performance would vary over the band but be degraded for most of the band. Figure 48 shows isolation reduction when ports three and four are terminated. If the designer were to take a look at figure 50 then he/she would say that there is an added benefit in having the shield line terminations as open circuits.

Figures 56-61 illustrate the isolation reduction vs. line spacing. This view of isolation reduction would allow a designer to see how much noise immunity would be gained if a shielded line under specific termination conditions were implemented for a specific frequency and the isolation reduction is viewed versus line spacing. An example in applying such a plot would be if a designer is working in a narrow band that is centered at $2.5 \mathrm{GHz}$, and has been given mechanical constraints for line spacing and has determined that they can be anywhere from $450 \%$ to $600 \%$. A look at figure 58 shows that the implementation of a shielded line at $450 \%$ spacing would enhance the noise immunity of the data bus by about $1 \mathrm{~dB}$ with the port on the shielded line adjacent to the transmitting line open circuited. The designer is better off staying with a dual line structure in this case. However, if the center frequency is moved down to $500 \mathrm{MHz}$, noise immunity is degraded by approximately $7.6 \mathrm{~dB}$ with the shield line ports open. At $5 \mathrm{GHz}$ noise immunity is severely degraded for all shield line termination conditions by approximately $14 \mathrm{~dB}$ with the shield line ports terminated in the line characteristic impedance. Therefore the variance of isolation gain is very large 
and is, in most application viewed best versus frequency and compared against the different termination conditions on the shielded line.

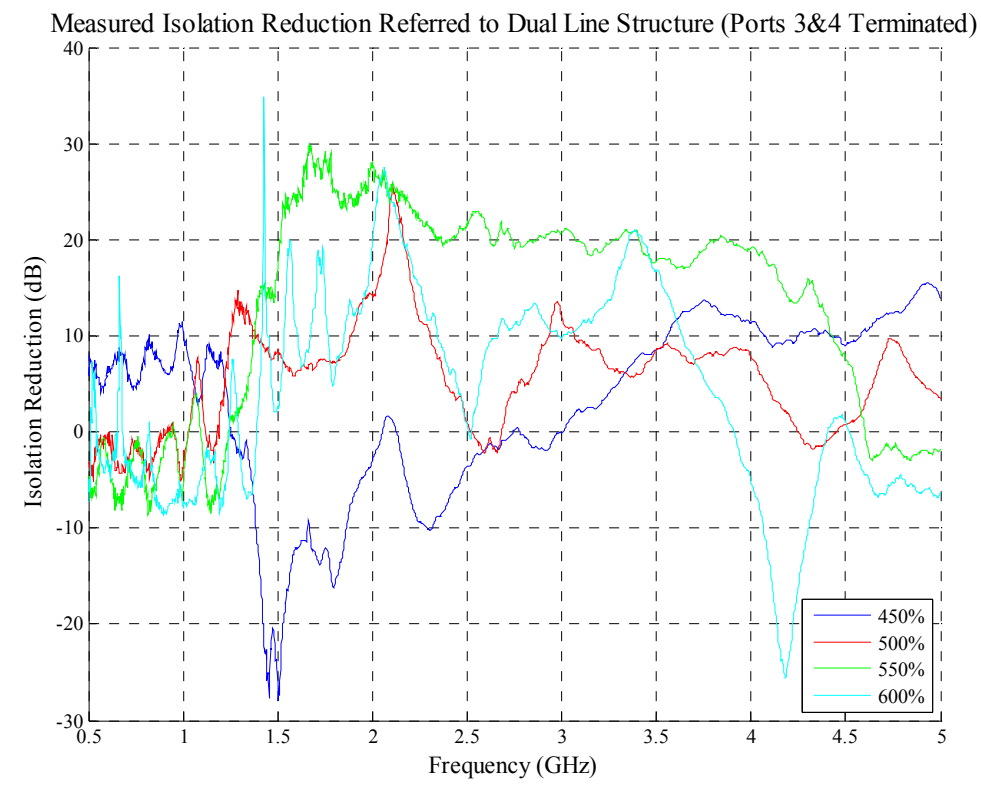

Figure 48. Measured isolation reduction vs. frequency referenced to dual line (ports $3 \& 4$ Terminated)

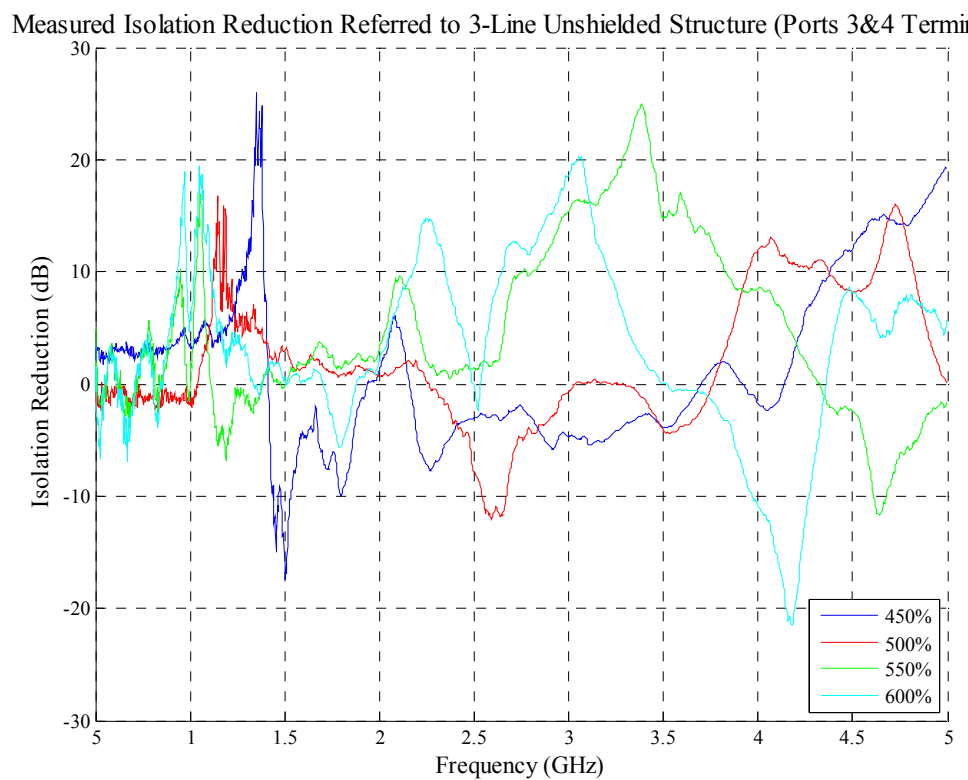

Figure 49. Measured isolation reduction vs. frequency referenced to three line (ports $3 \& 4$ Terminated) 


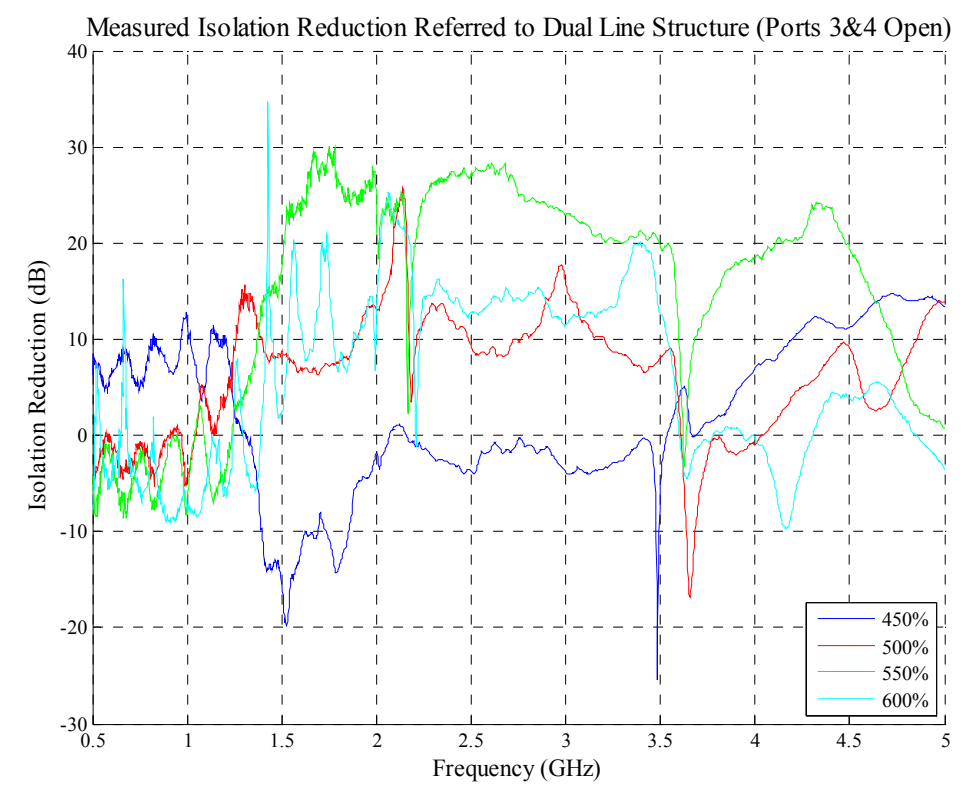

Figure 50. Measured isolation reduction vs. frequency referenced to dual line (ports $3 \& 4$ open)

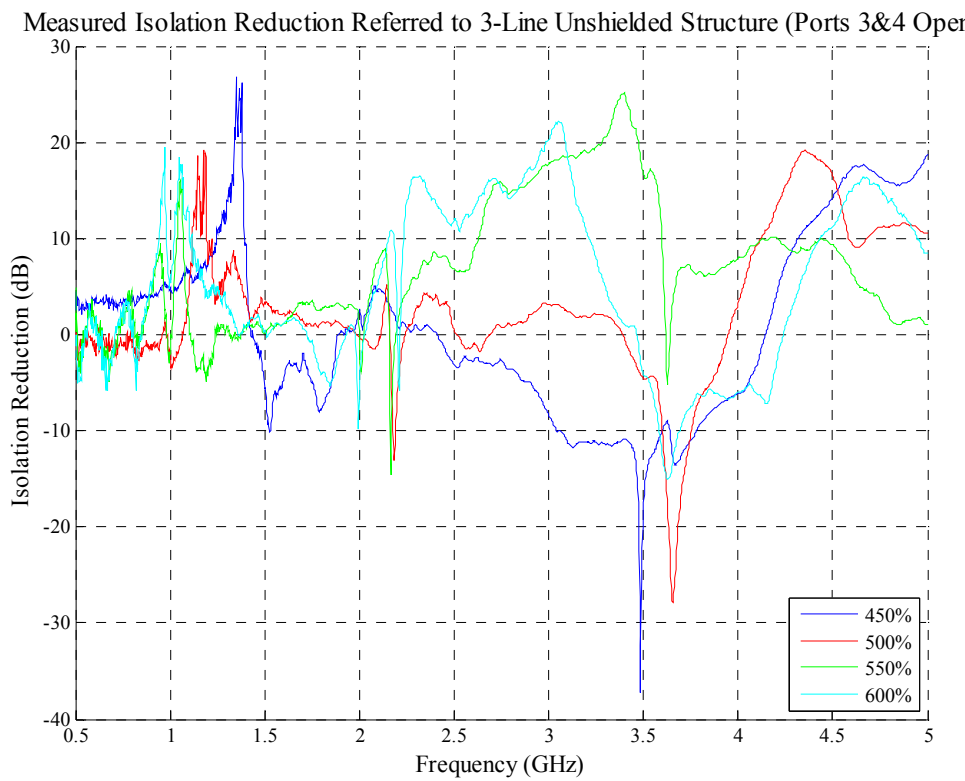

Figure 51. Measured isolation reduction vs. frequency referenced to three line (ports $3 \& 4$ open) 


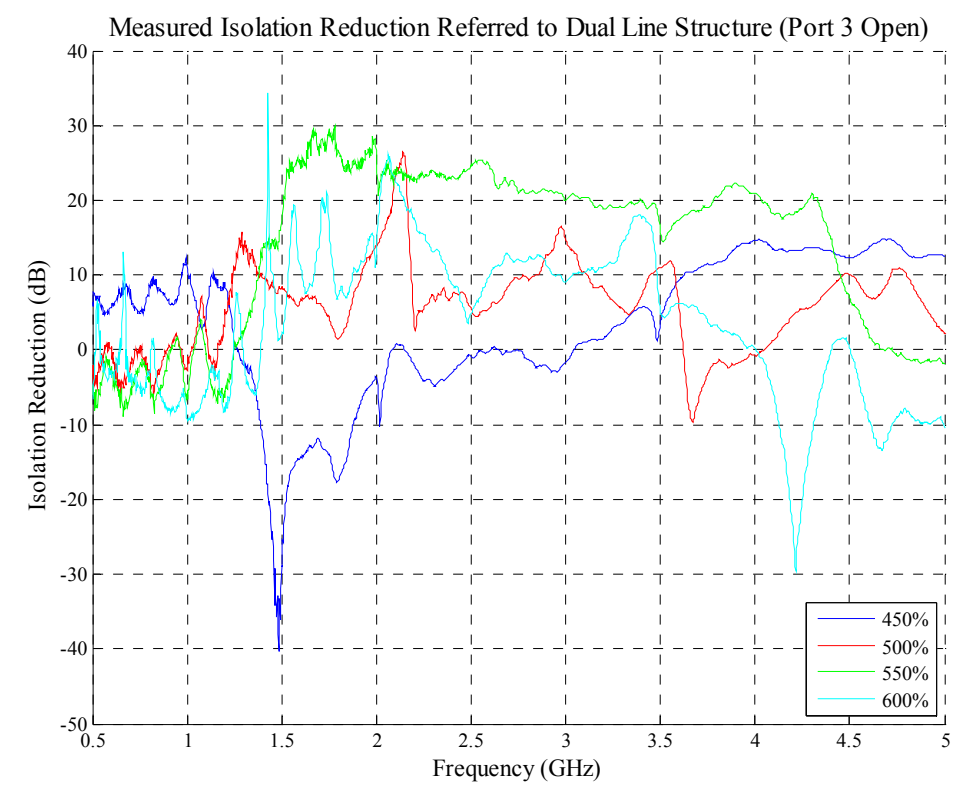

Figure 52. Measured isolation reduction vs. frequency referenced to dual line (port 3 open)

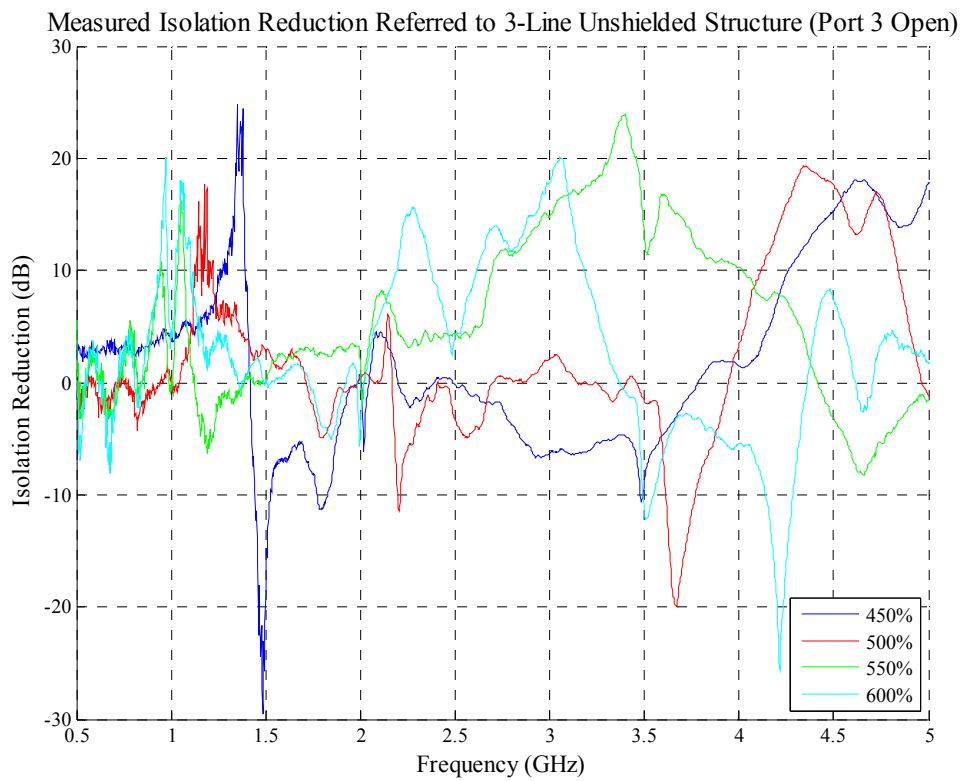

Figure 53. Measured isolation reduction vs. frequency referenced to three line (port 3 open) 


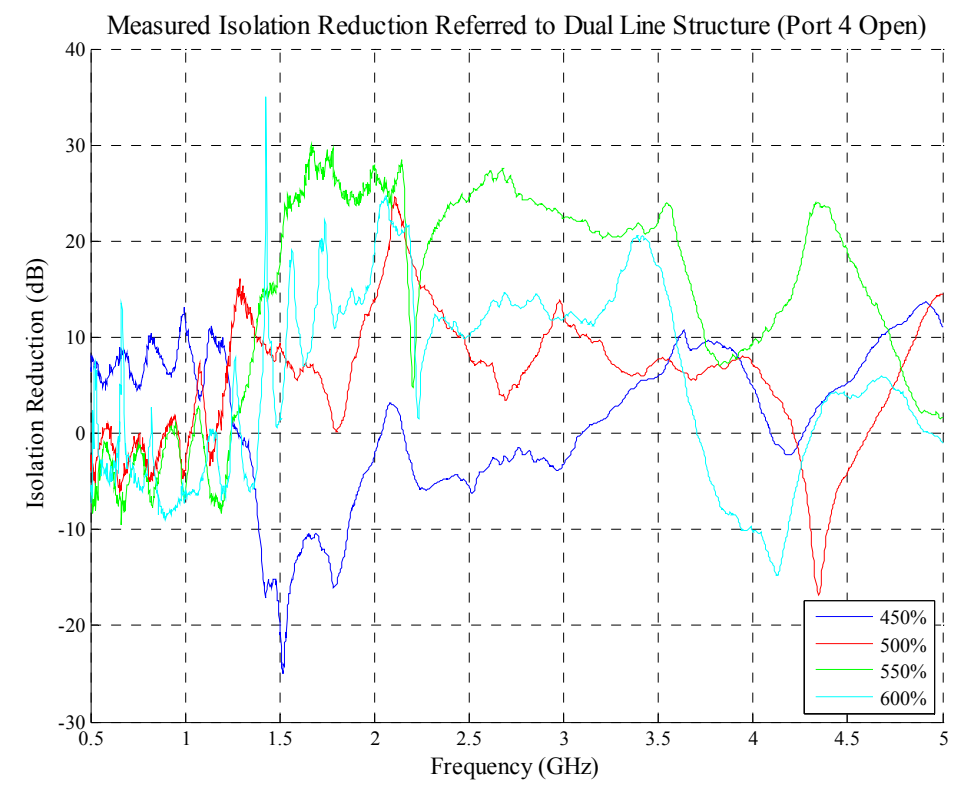

Figure 54. Measured isolation reduction vs. frequency referenced to dual line (port 4 open)

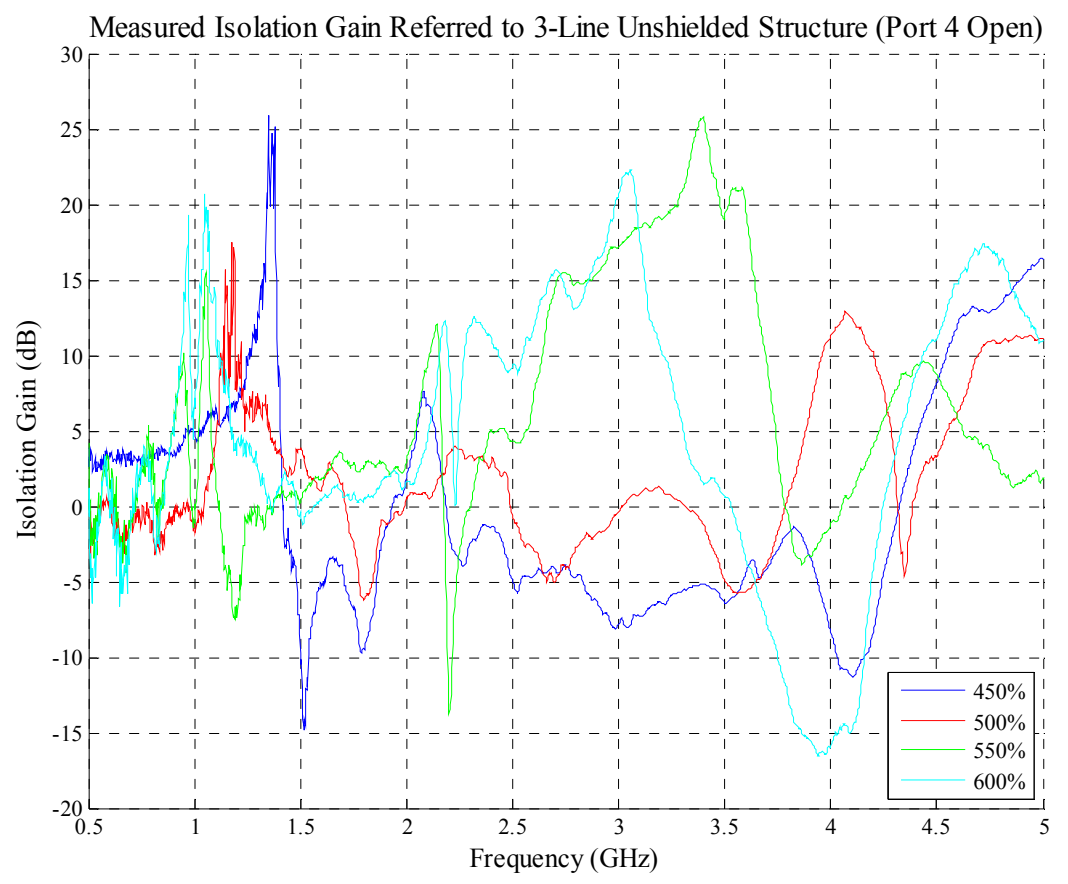

Figure 55. Measured isolation reduction vs. frequency referenced to three line (port 4 open) 


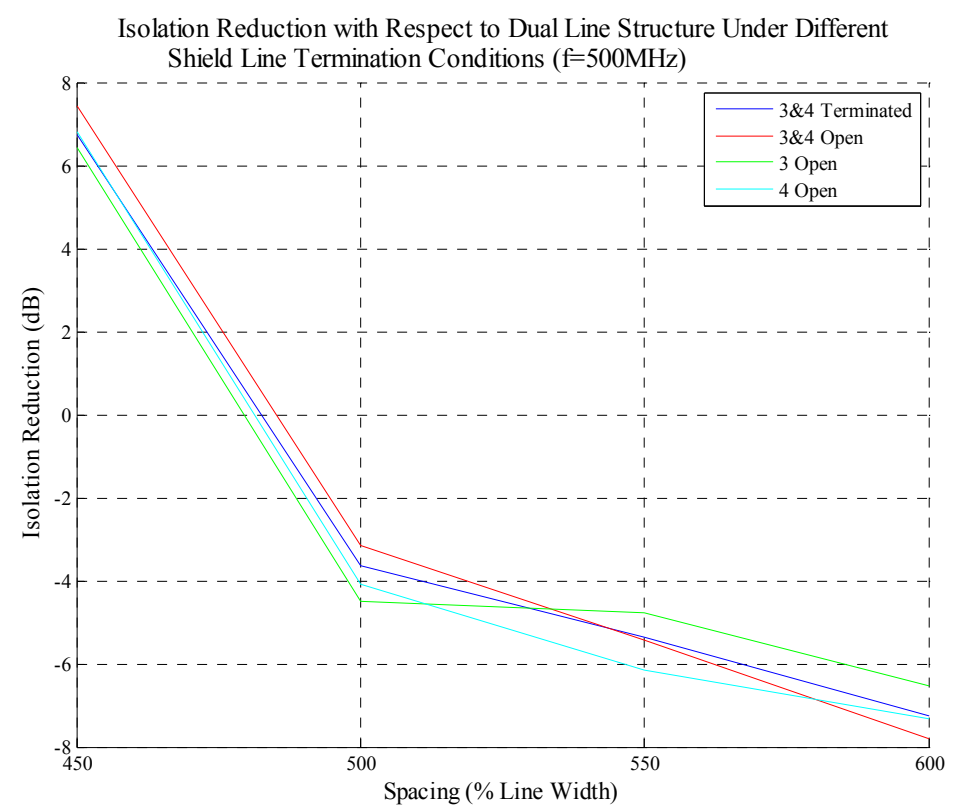

Figure 56. Measured isolation reduction vs. line spacing referenced to dual line structure $(f=500 \mathrm{MHz})$

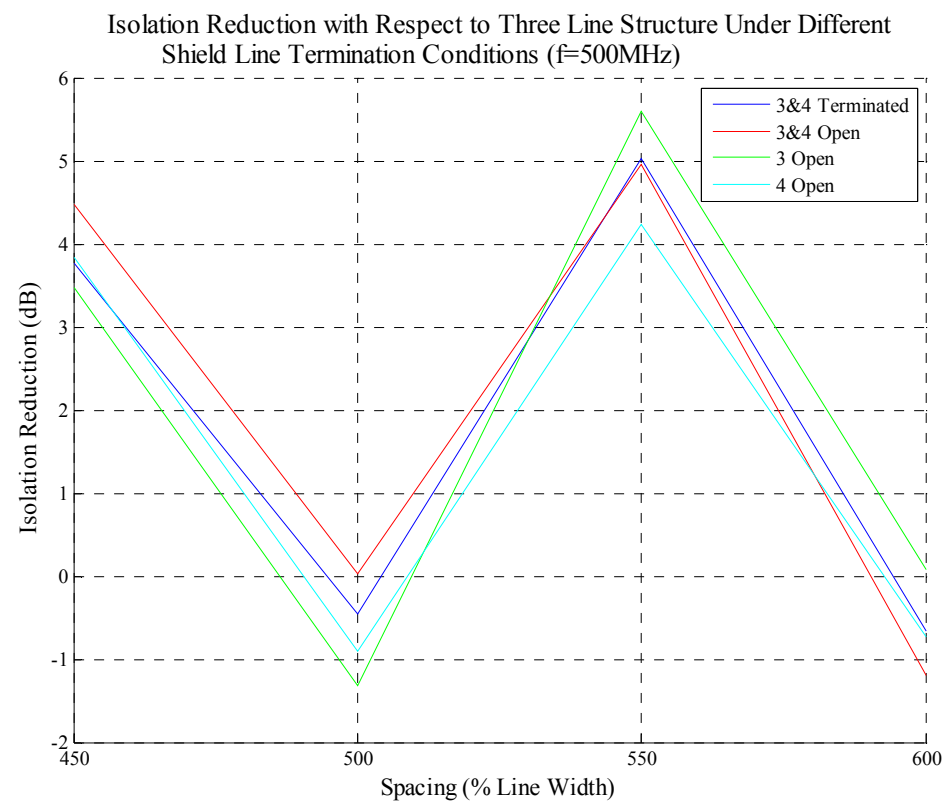

Figure 57. Measured isolation reduction vs. line spacing referenced to three line structure $(f=500 \mathrm{MHz})$ 


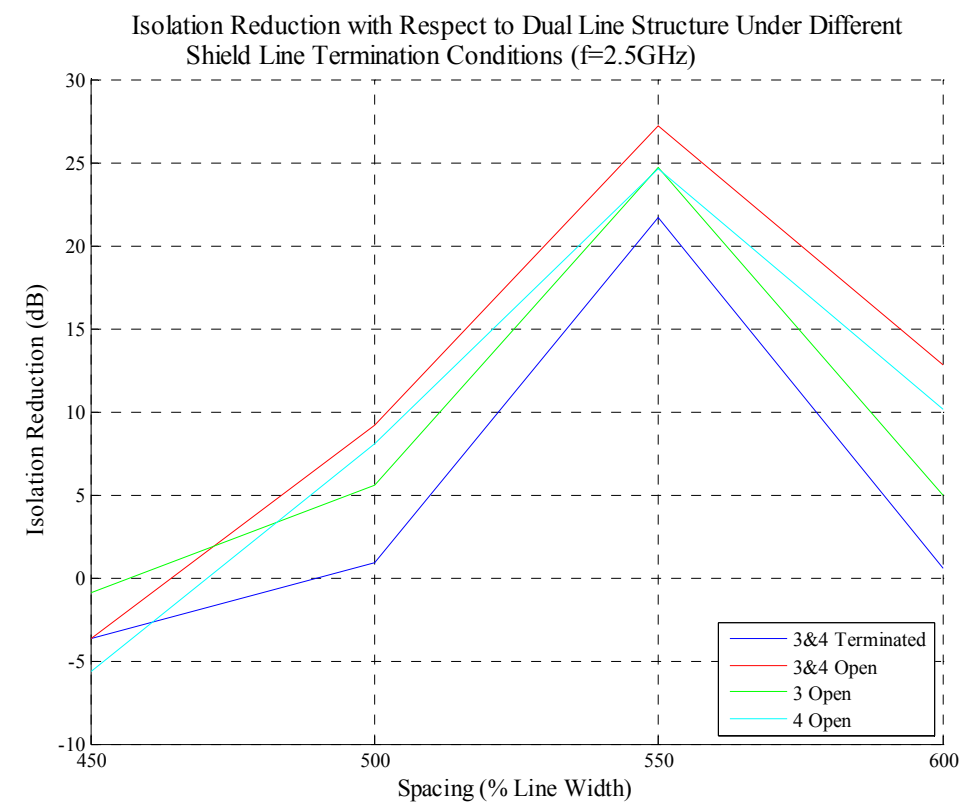

Figure 58. Measured isolation reduction vs. line spacing referenced to dual line structure $(f=2.5 \mathrm{GHz})$

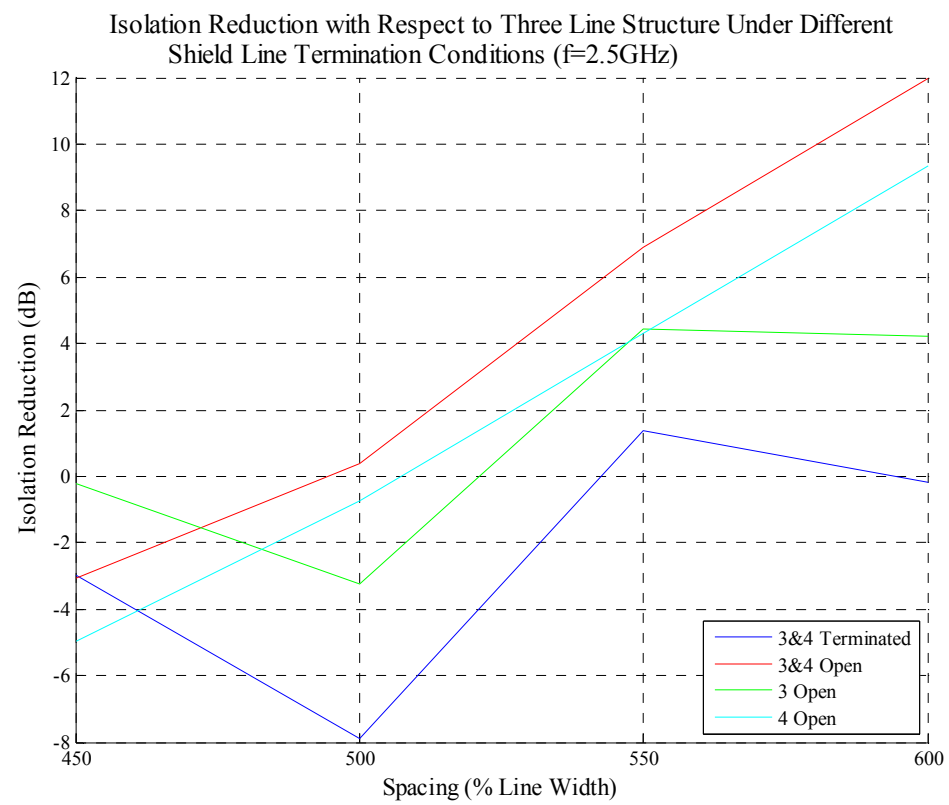

Figure 59. Measured isolation reduction vs. line spacing referenced to three line structure $(f=2.5 \mathrm{GHz})$ 


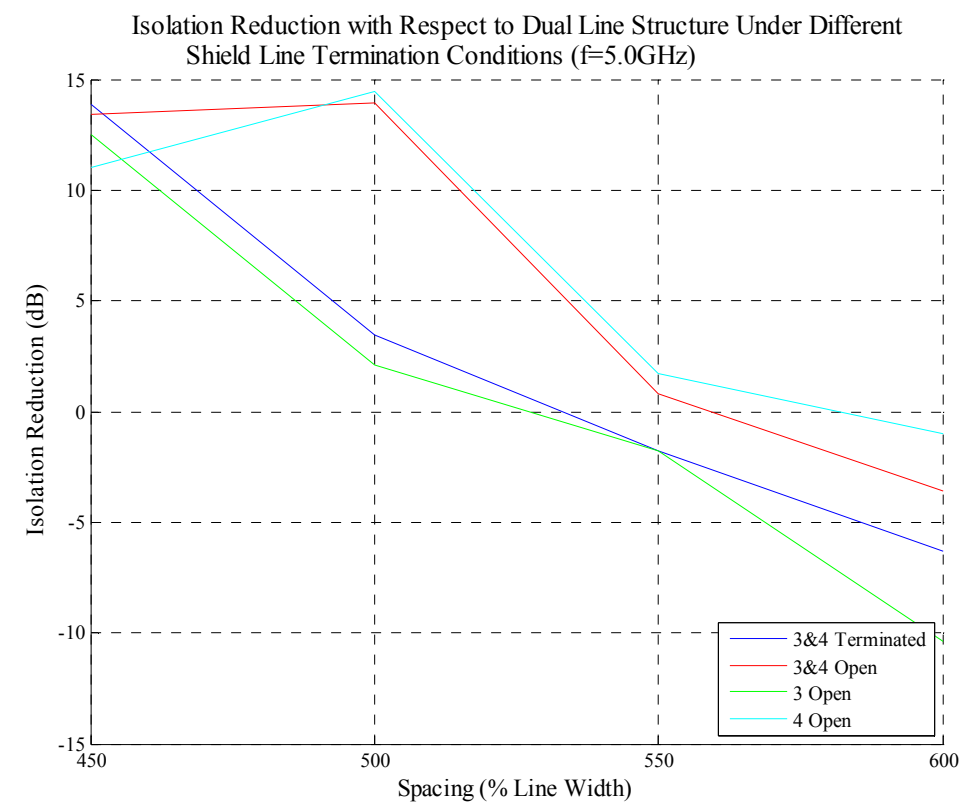

Figure 60. Measured isolation reduction vs. line spacing referenced to dual line structure $(f=5.0 \mathrm{GHz})$

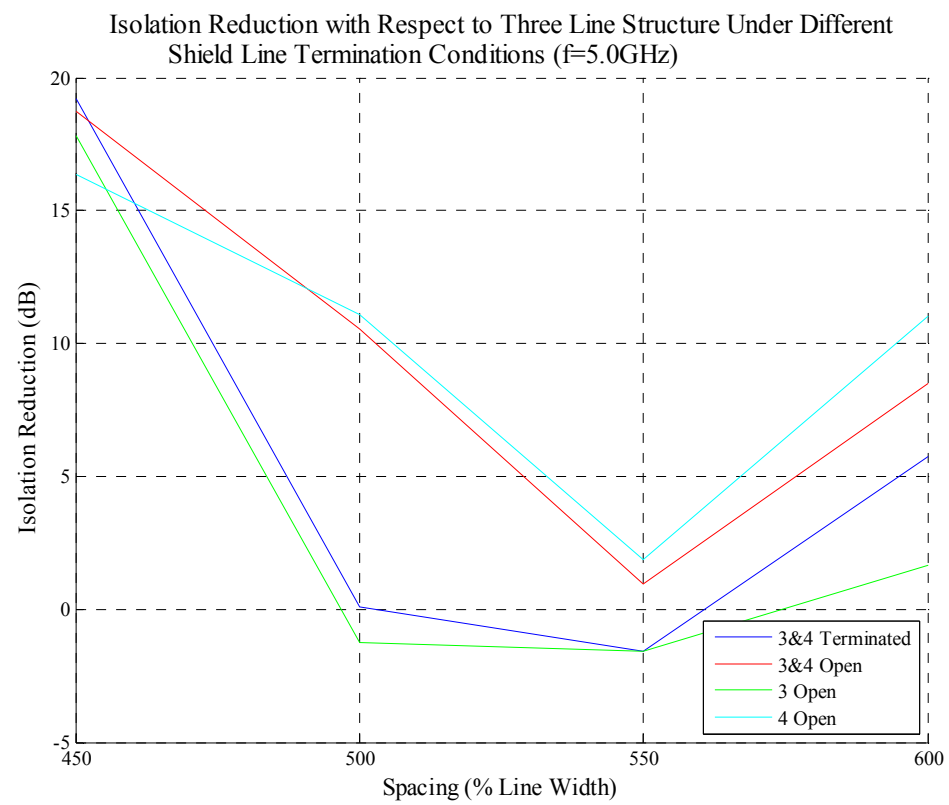

Figure 61. Measured isolation reduction vs. line spacing referenced to three line structure $(f=5.0 \mathrm{GHz})$ 


\section{Chapter 6}

\section{INTRINSIC ERROR BETWEEN DOMAINS}

In this chapter, we will discuss errors introduced into the system and why there is not a $100 \%$ correlation between the results of the digital domain and the real world measurements. The coaxial to microstrip transition is a discontinuity [25] that creates reflections, impedance mismatches, at the source and load end of the lines.

Finite substrate dimensions have an effect on the capacitance of the microstrip lines. Baginski et al. [26] showed that microstrip capacitance versus distance from board edge was at a minimum at the board edge and increased in a 1-e fashion toward its infinite substrate value. For substrates with $\varepsilon_{\mathrm{r}}$ between four and five the capacitance approached its infinite substrate self-capacitance value at approximately one line width from the edge measured from the nearest strip edge. The $600 \%$ structure has a distance, in line widths, of approximately three. This would lead one to postulate that the self-capacitance of all strip conductors are approximately equal because line spacing only decreases from $600 \%$ thus giving greater confidence that the strip conductor has reached its infinite substrate self-capacitance value.

Simulation environments provide the convenience of being able to completely tune out and ignore external effects such as incident electromagnetic waves. The ability to tune out external factors is invaluable for understanding the consequences of altered physical and/or electrical properties of a structure. A user of these environments must always be aware that the model simulated is limited by the amount of detail that the user can put in the model and that there are unpredictable sources of noise that will corrupt measurement results. The measured results showed a large external bombardment from a wireless router operating at 2.4GHz. An extra $4 \mathrm{~mW}$ were induced into the structure. Modeling in HFSS or Sonnet allows you to ignore these factors. This external interference was not detected in HFSS simply because I did not include it.Percentage error between domains can reveal instrument calibration error, miscalculation in simulation depending on what domain is used as reference and large percentage errors can be interpreted as external bombardment of electromagnetic radiation. The percentage error referenced to the simulated data will be expressed as, 


$$
\% \text { Error }=\frac{\left|S_{61}\right|_{\text {Measured }}-\left|S_{61}\right|_{\text {Simulated }}}{\left|S_{61}\right|_{\text {Simulated }}} \times 100 \%
$$

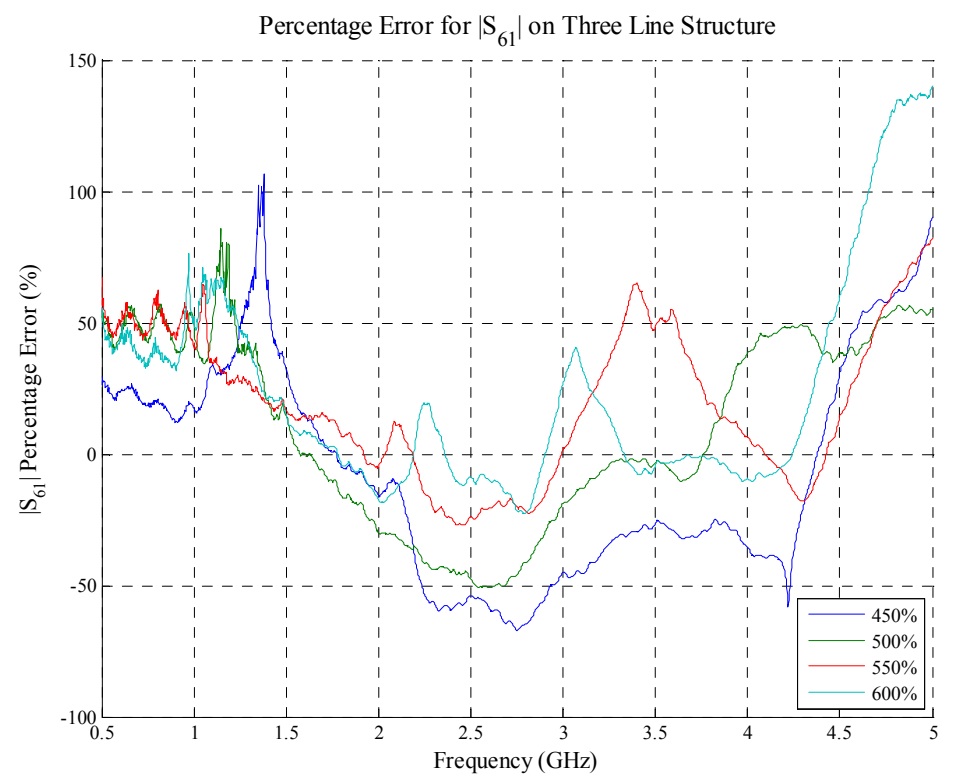

Figure 62. Percentage Error for three line structure

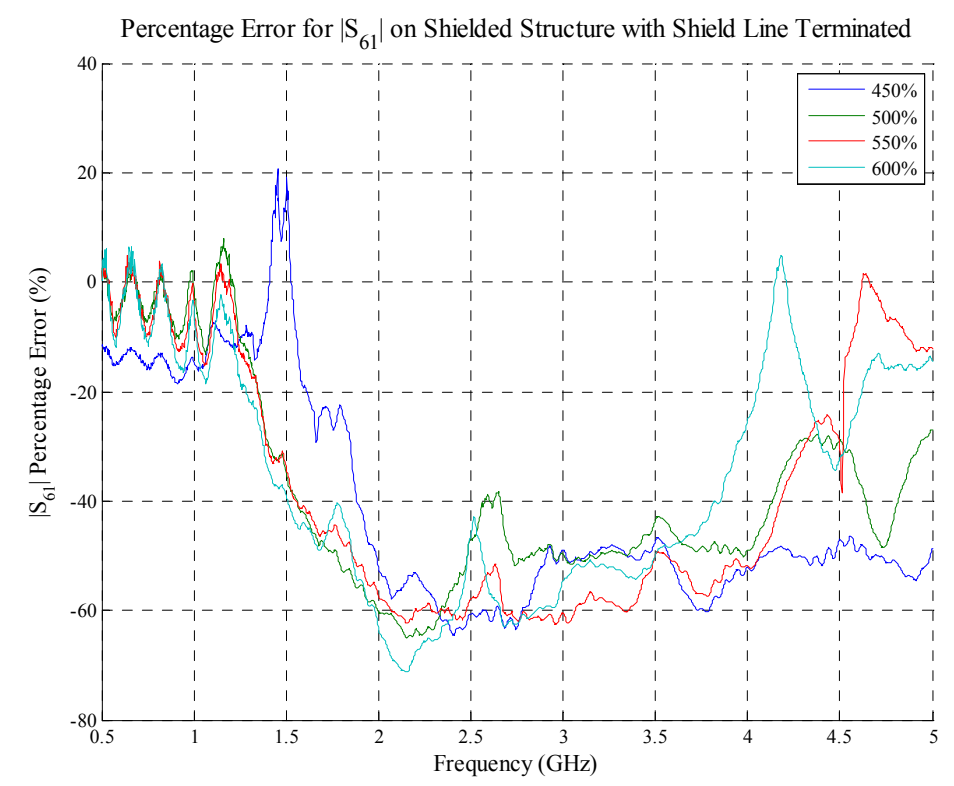

Figure 63. Percentage Error for terminated shielded structure 


\section{ChAPTER 7}

\section{CONCLUSIONS AND FUture WORK}

In this thesis, the coupling mechanisms for microstrip were investigated qualitatively in terms of their field interactions. Simulations were carried out in Sonnet's finite element method solver in order to quantify the coupling between closely spaced transmission lines in a microstrip, stripline, and microstrip with dielectric overlay structure. The results show that the stripline structure intrinsically possesses the greatest noise immunity because of its encapsulated form where the ground plane can serve as a reflector for incident electromagnetic radiation and the results for crosstalk after implementation of the shielding line only serves to degrade noise immunity immensely as frequency increases. Microstrip with dielectric overlay exhibited the same isolation characteristics as stripline but to a lesser degree. Isolation on the unshielded microstrip with dielectric overlay was not as high as stripline and, like stripline, did suffer a degradation in crosstalk performance where the crosstalk continued to increases constantly as frequency increases. The greatest benefit to the shielding technique was seen in the microstrip structure. One example of this success was in figure 16 the crosstalk performance is clearly improved by approximately $5 \mathrm{~dB}$ for the majority of the frequency band of interest.

Other factors such as decreased substrate thickness and greater line width to substrate thickness ratio, greater spacing to line width ratios all lower crosstalk considerably and

HFSS provided the means to simulate larger domains and the actual microstrip structure that was to be fabricated with great ease. Accuracy between measurements and simulation suffered greatly due to external interference. Though the accuracy suffered a loss the actual isolation reduction was measured and shown to be negative when referenced to both three line and dual line structures which indicated that the shield line is a technique that works in certain frequency bands depending on the structure and termination conditions.

Three variations of microstrip structures were fabricated in house in order to validate the findings of the simulations in HFSS and to also have a reference for the isolation gain calculations. Measurements were taken with the shielded structures under different termination conditions for the shielded line. These variations on termination did have an effect on the overall crosstalk response. Depending on the 
application, after the designer is able to conclude that the shield line would enhance the noise immunity, the appropriate terminating condition for the shielded line would be applied. The gain in noise immunity is given as the isolation gain.

Errors were expected to be found in the coaxial to microstrip transition, in the finite dimensions of the substrate, line width variance that creates small impedance mismatches and reflections. The presence of other devices in the laboratory influenced the outcome of the measurements greatly. $4 \mathrm{~mW}$ of power were absorbed by the device under test at $2.4 \mathrm{GHz}$ because of the router present in the laboratory. Errors between the virtual domain and reality were found to be mainly the lack of detail in the model and the absence of the incident electromagnetic radiation.

In the future, it would be beneficial to further investigate the shielded lines by varying the width of the shield line, varying the spacing between vias, apply effective through hole plating for the short circuits to minimize the height difference and altered charge distributions found when using copper wire for short circuits. 


\section{BIBLIOGRAPHY}

[1] Gawon Kim, Eakhwan Song, Jiseong Kim, and Joungho Kim, "Precise Analysis and Modeling of FarEnd Crosstalk and Far-End Crosstalk Saturation Using Mode Analysis in Coupled Microstrip Lines," Electrical Design of Advanced Packaging \& Systems Symposium, (C) 2011 IEEE. , pp. 1-4, December 2009.

[2] M. Fletcher, A. Abel, P.F. Wahid, and M.A. Belkerdid, "Modeling of Crosstalk in Coupled Microstrip Lines," Southeastcon '88., IEEE Conference Proceedings, (C 2011 IEEE. , pp. 506-510, April 1988.

[3] G. Pourparviz, G. Goforth, P.F. Wahid, and M.A. Belkerdid, "Frequency Domain Analysis of Crosstalk in Coupled Microstrip Lines," Southeastcon'88., IEEE Conference Proceedings, (C) 2011 IEEE. , pp. 11-13, April 1988.

[4] D.S. Gao, A.T. Yang, and S.M. Kang, "Accurate Modeling and Simulation of Parallel Interconnection in High-Speed Integrated Circuits," Circuits and Systems, 1988., IEEE International Symposium on, (C) 2011 IEEE. , vol. 3, pp. 2105-2108, June 1988.

[5] F. Sellberg, "Simple Determination of All Capacitances for a Set of Parallel Microstrip Lines," Microwave Theory and Techniques, IEEE Transactions on, (C) 2011 IEEE. , vol. 46, no. 2, pp. 195-198, February 1998.

[6] M.-S. Lin, "Measured Capacitance Coefficients of Multiconductor Microstrip Lines with Small Dimensions," Components Hybrids, and Manufacturing Technology, IEEE Transactions on, (C 2011 IEEE. , vol. 13, no. 4, pp. 1050-1054, December 1990.

[7] David M. Pozar, Microwave Engineering. Hoboken: John Wiley \& Sons, Inc., 2005.

[8] F. Romeo and M. Santomauro, "Time-Domain Simulation of n Coupled Transmission Lines," Microwave Theory and Techniques, IEEE Transactions on, (C) 2011 IEEE. , vol. 35, no. 2, pp. 131-137, February 1987.

[9] R. Senthinathan, J. Prince, and M. Scheinfein, "Characteristics of Coupled Buried Microstrip Lines by Modeling and Simulation," Components, Hybrids, and Manufacturing Technology, IEEE Transactions on, (C) 2011 IEEE. , vol. 10, no. 4, pp. 604-611, December 1987.

[10] A.M. Abbosh, "Analytical Closed-Form Solutions for Different Configurations of Parallel-Coupled Microstrip Lines," Microwaves, Antennas \& Propagation, IET, (C 2011 IEEE. , vol. 3, no. 1, pp. 137147, February 2009.

[11] E. Bogatin, "Design Rules for Microstrip Capacitance," IEEE Transactions on Components, Hybrids, and Manufacturing Technology, (C) 2011 IEEE. , vol. 11, no. 3, pp. 253-259, September 1988.

[12] Huaian Zhou, Zhengwei Du, and Ke Gong, "A Closed Form Analytical Formulation for the Coupling of an External Electromagnetic Wave with a Microstrip Lines," Microwave, Antenna, Propagation and EMC Technologies for Wireless Communications, 2005. MAPE 2005. IEEE International Symposium on, (C 2011 IEEE. , vol. 1, pp. 700-703, August 2005.

[13] M. Leone and L. Hermann, "On the Coupling of an External Electromagnetic Field to a Printed Circuit Board Trace," Electromagnetic Compatibility, IEEE Transactions on, (C) 2011 IEEE. , vol. 41, no. 4, pp. 418-424, 1999. 
[14] T. Bryant and J. Weiss, "Parameters of Microstrip Transmission Lines and Coupled Pairs of Microstrip Lines," Microwave Theory Techniques, IEEE Transactions on, (C) 2011 IEEE. , vol. 16, pp. 1021-1027, 1968.

[15] R. Jansen, "High-Speed Computation of Single and Coupled Microstrip Parameters Including Dispersion, HigherOrder Modes, Loss, and Finite Strip Thickness," Microwave Theory Techniques, IEEE Transactions on, (C) 2011 IEEE. , vol. 26, pp. 75-82, 1978.

[16] M.E. Hellman and I. Palocz, "The Effect of Neighboring Conductors on the Currents and Fields in Plane Parallel Transmission Lines," Microwave Theory and Techniques, IEEE Transactions on, (C) 2011 IEEE. , vol. 17, no. 5, pp. 254-259, May 1969.

[17] K.G. Black and T.J. Higgins, "Rigorous Determination of the Parameters of Microstrip Transmission Lines," Microwave Theory and Techniques, IRE Transactions on, (C) 2011 IEEE. , vol. 3, pp. 93-113, March 1955.

[18] M.E. Goldfarb and R.A. Pucel, "Modeling Via Hole Grounds in Microstrip," IEEE Microwave and Guided Wave Letters, (C) 2011 IEEE. , vol. 1, no. 6, pp. 135-137, June 1991.

[19] T. Kasuga and H. Inoue, "A Study on Suppression of Crosstalk Between Parallel Transmission Lines at High Frequency Band," Electromagnetic Compatibility, 2007. EMC 2007. IEEE International Symposium on, C 2011 IEEE. , pp. 1-6, July 2007.

[20] V. Ungvichian and M.B. Kopp, "Crosstalk in Coupled Microstrip Lines due to Substrate Permittivity and S/h Ratios," Southcon/94. Conference Record, (C) 2011 IEEE. , pp. 301-304, March 1994.

[21] B. Drnovsek, J. Koselj, A. Znidarsic, and V.B. Bregar, "Study of Coupling on Parallel Microstrip Lines Due to Magnetic-Loaded Absorber Sheet," Magnetics, IEEE Transactions on, (C) 2011 IEEE. , vol. 44, no. 11, pp. 38013804, November 2008.

[22] M. Kazerooni, A. Cheldavi, and M. Kamarei, "Crosstalk and Electromagnetic Interference Noise Investigation for a Coupled Pair of Microstrip Lines with a Break in Ground Structure," Microwaves, Antennas \& Propagation, IET, (C) 2011 IEEE. , vol. 4, no. 9, pp. 1336-1346, September 2010.

[23] S. Malisuwan and V. Ungvichian, "A Study of Crosstalk Applied to Ultra-High-Speed Parallel Lossy Microstrip and Strip Lines with Shielding Effectiveness," Electromagnetic Compatibility, 1999 International Symposium on, (C) 2011 IEEE. , pp. 742-745, 1999.

[24] Bill Oldfield, Jim Rautio, Jim Merrill, Ted Miracco, and Paul Draxler, "Simple Microstrip Structures Calculated vs Measured," 37th ARFTG COnference Digest-Spring, (C) 2011 IEEE. , pp. 10-20, 1991.

[25] C. Capsalis, C.P. Chronopoulous, and N.K. Uzunoglu, "A Rigorous Analysis of a Coaxial to Shielded Microstrip Line Transition," Microwave Theory and Techniques, IEEE Transactions on, (C) 2011 IEEE. , vol. 37, no. 7, pp. 1091-1098, July 1989.

[26] M.E. Baginski, L.S. Riggs, F.J. German, and G.W., Jr. Jarriel, "Finite-Element Analysis of Microstrip Systems in the Vicinity of a Substrate Edge and Approximate Formulas for Capacitance," Electromagnetic Compatibility, IEEE Transactions on, (C) 2011 IEEE. , pp. 404-408, 1994.

[27] T. Hiraoka, Kai Ding, and Jui-Pang Hsu, "Calculations of Characteristic Impedance for Parallel Coupled Stripline Based on Eigenmode Analysis and Equivalent Network," Microwave Symposium Digest, 2009. MTT '09. IEEE MTT-S International, (C) 2011 IEEE. , pp. 269-272, June 2009. 


\section{APPENDICES}

Appendix A. Permissions

\section{A. Copyright Permission Statement from IEEE}

1) In the case of textual material (e.g., using short quotes or referring to the work within these papers) users must give full credit to the original source (author, paper, publication) followed by the IEEE copyright line (c) 2011 IEEE.

2) In the case of illustrations or tabular material, we require that the copyright line $\mathbb{C}$ [Year of original publication] IEEE appear prominently with each reprinted figure and/or table.

3) If a substantial portion of the original paper is to be used, and if you are not the senior author, also obtain the senior author's approval.

B. United States Code, Title 17, Chapter $1, \S 107$

Notwithstanding the provisions of sections $\underline{106}$ and $\underline{106 \mathrm{~A}}$, the fair use of a copyrighted work, including such use by reproduction in copies or phonorecords or by any other means specified by that section, for purposes such as criticism, comment, news reporting, teaching (including multiple copies for classroom use), scholarship, or research, is not an infringement of copyright. In determining whether the use made of a work in any particular case is a fair use the factors to be considered shall include-

(1) the purpose and character of the use, including whether such use is of a commercial nature or is for nonprofit educational purposes;

(2) the nature of the copyrighted work;

(3) the amount and substantiality of the portion used in relation to the copyrighted work as a whole; and

(4) the effect of the use upon the potential market for or value of the copyrighted work.

The fact that a work is unpublished shall not itself bar a finding of fair use if such finding is made upon consideration of all the above factors. 


\section{Appendix B. Measured Data Figures}
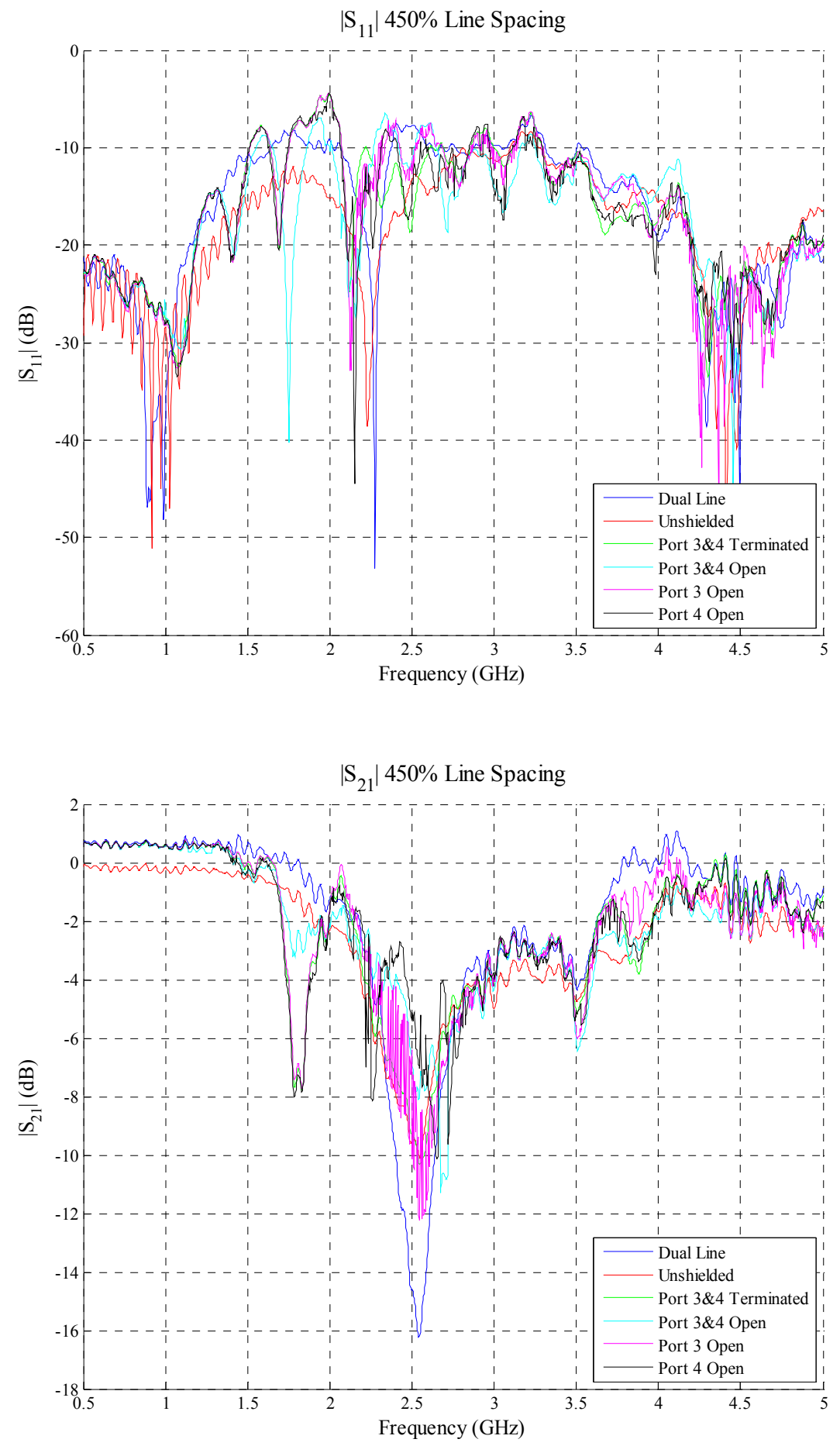

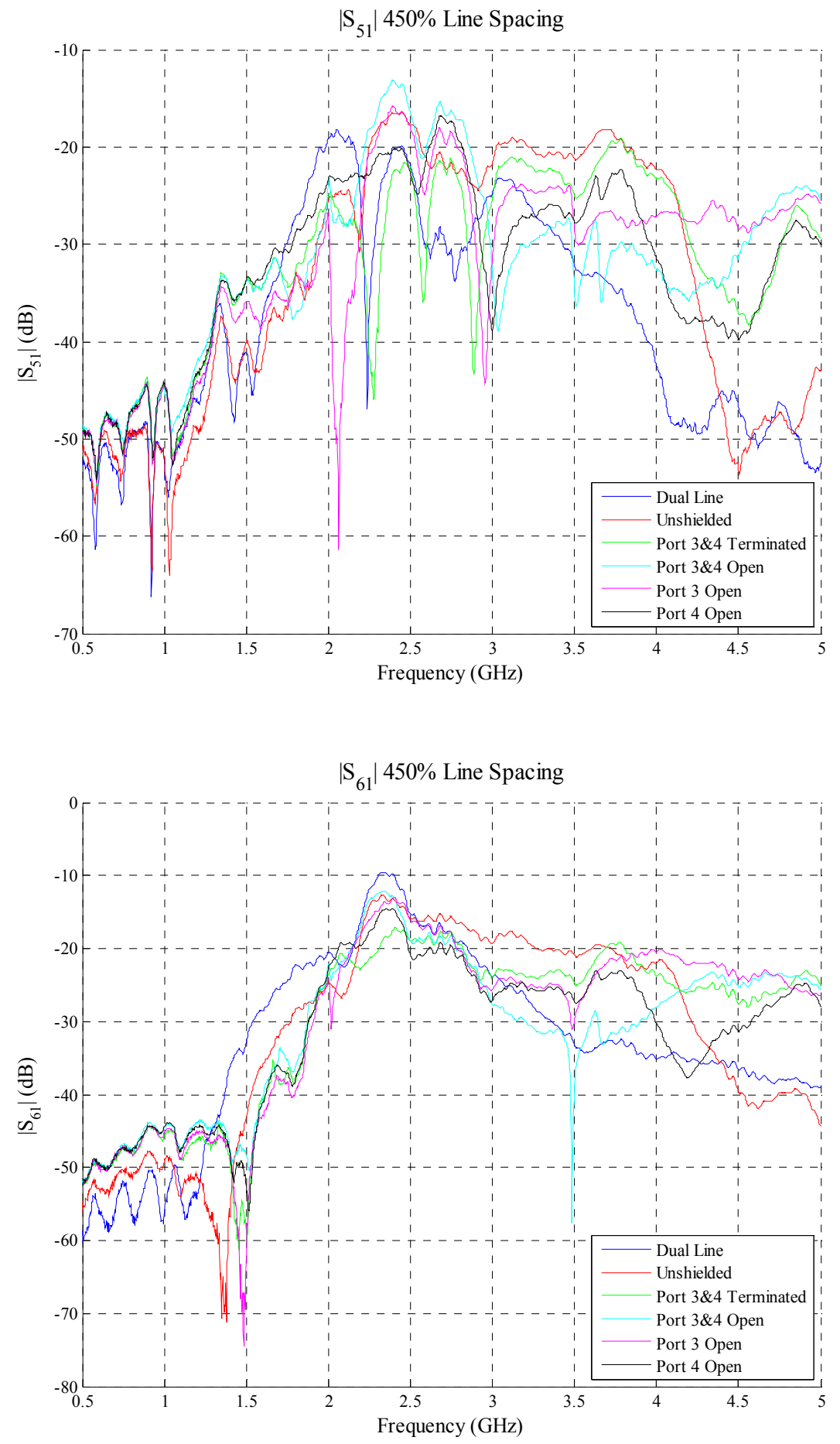

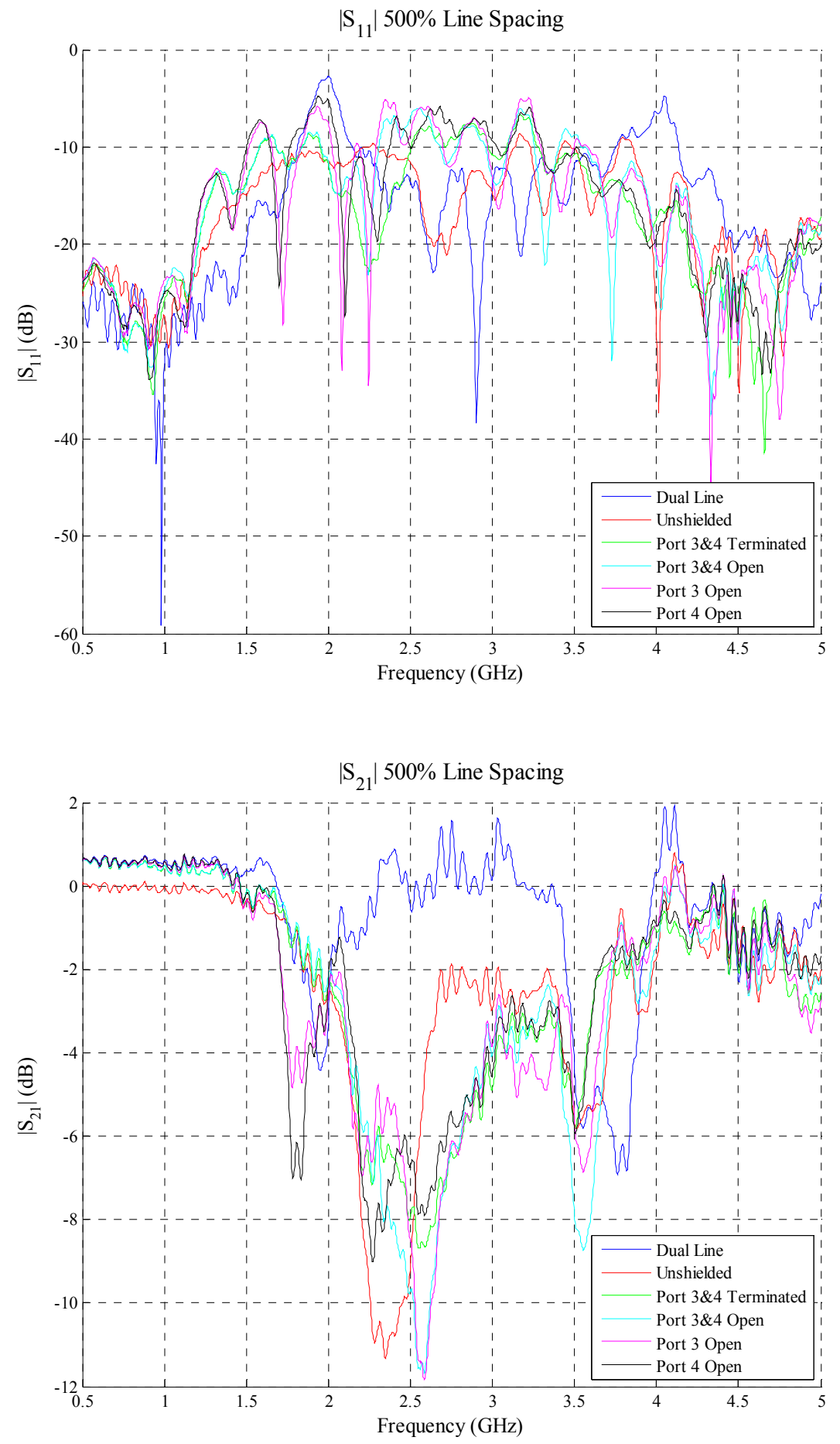

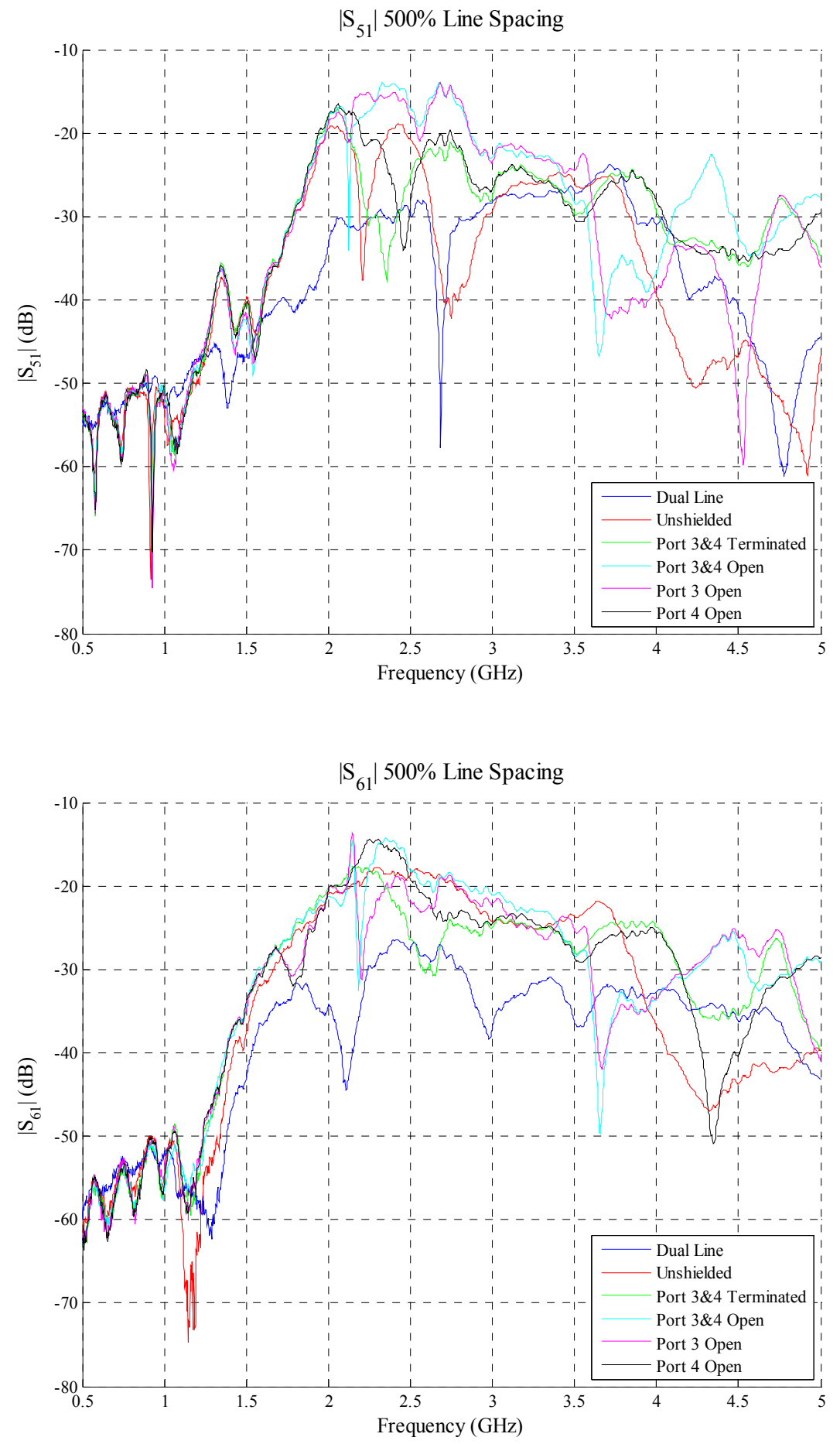

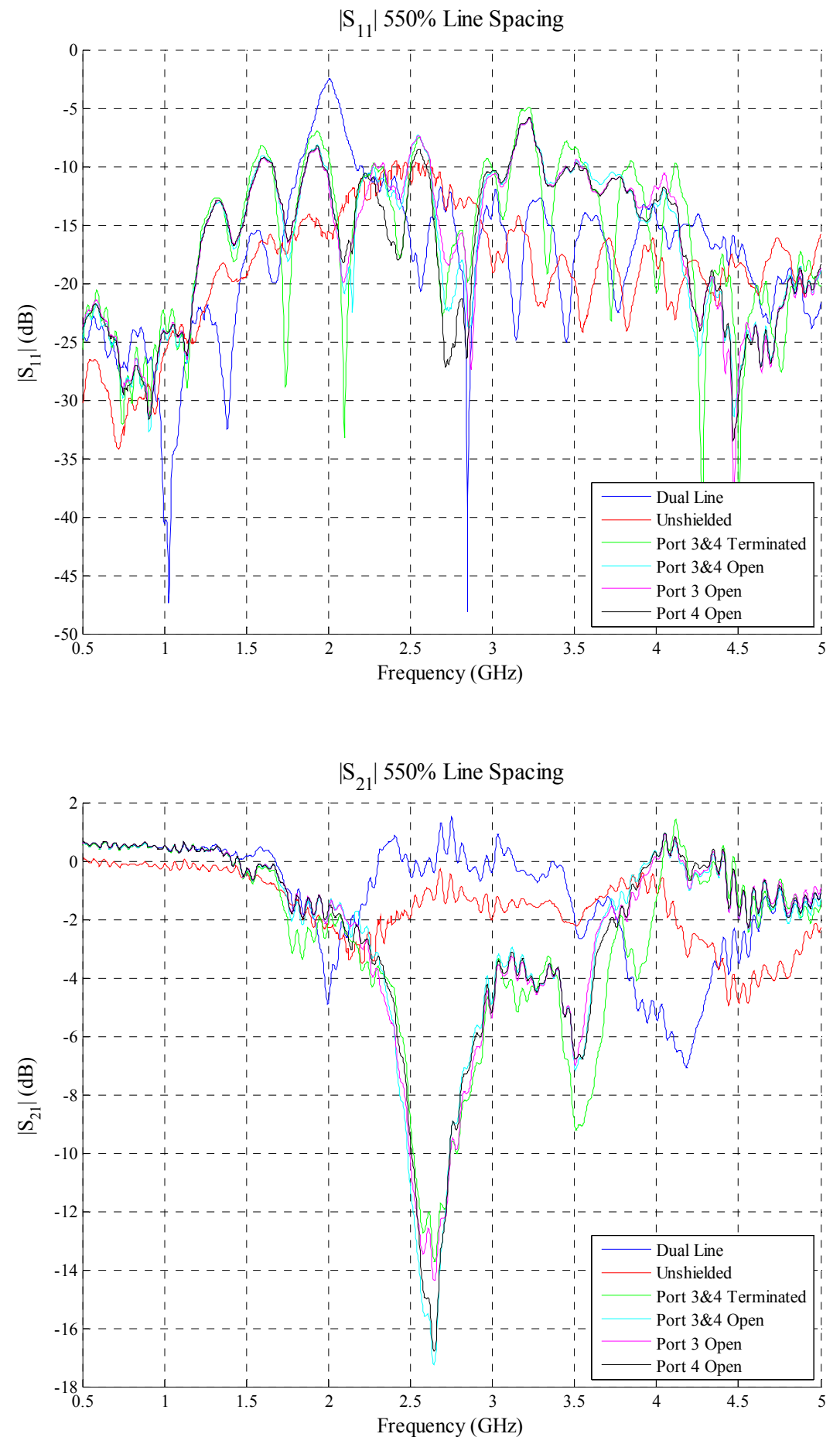

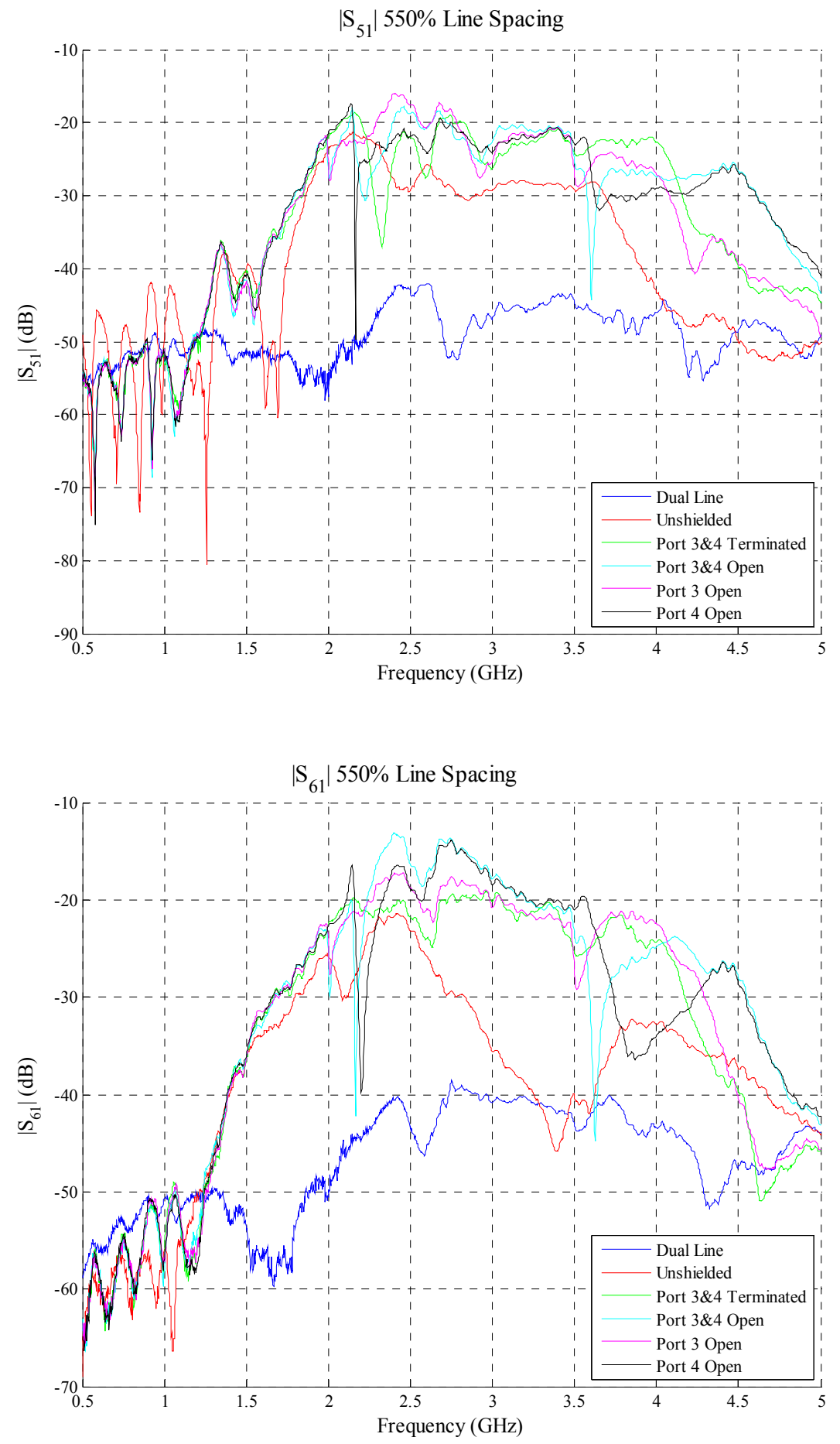

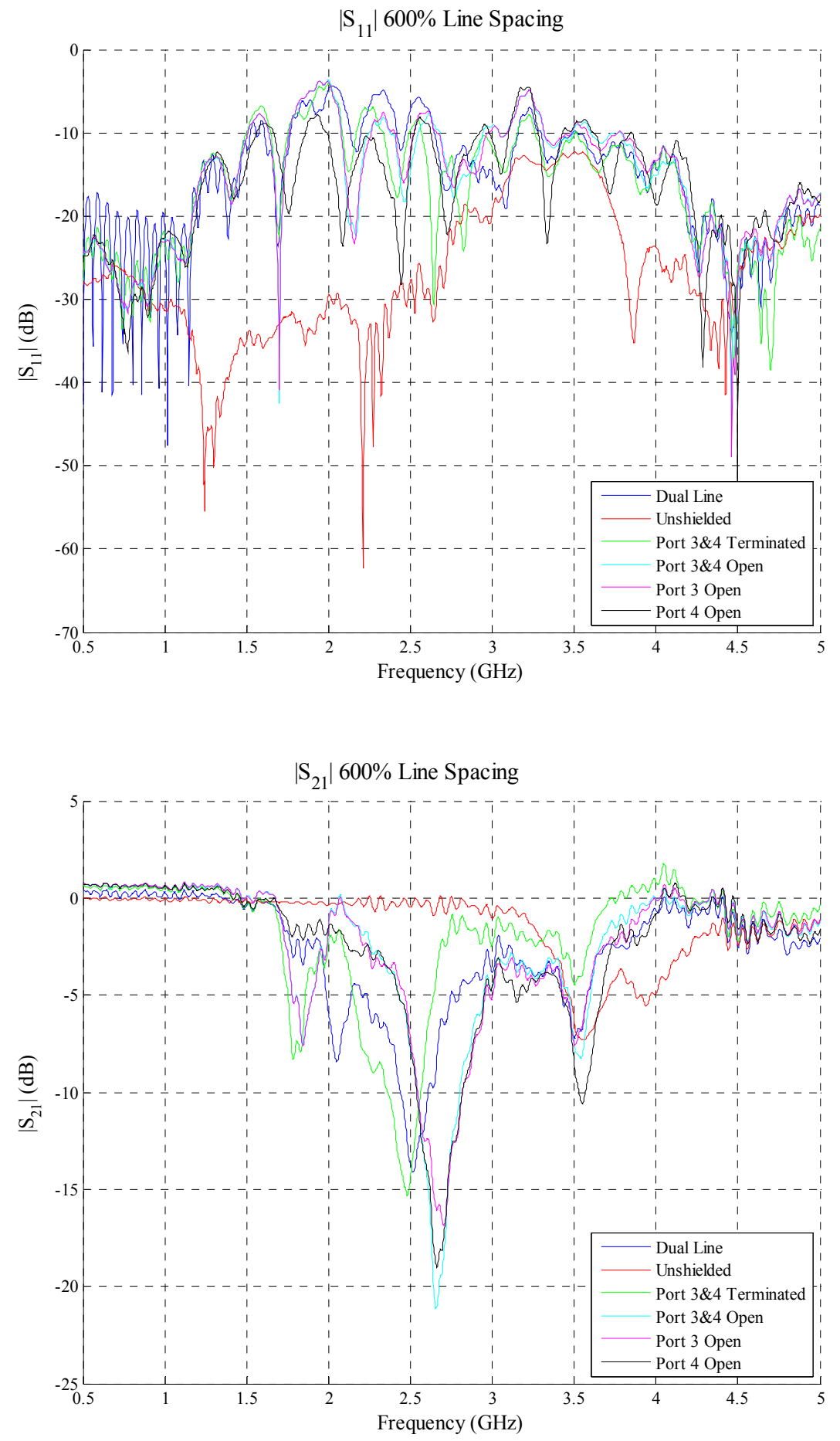

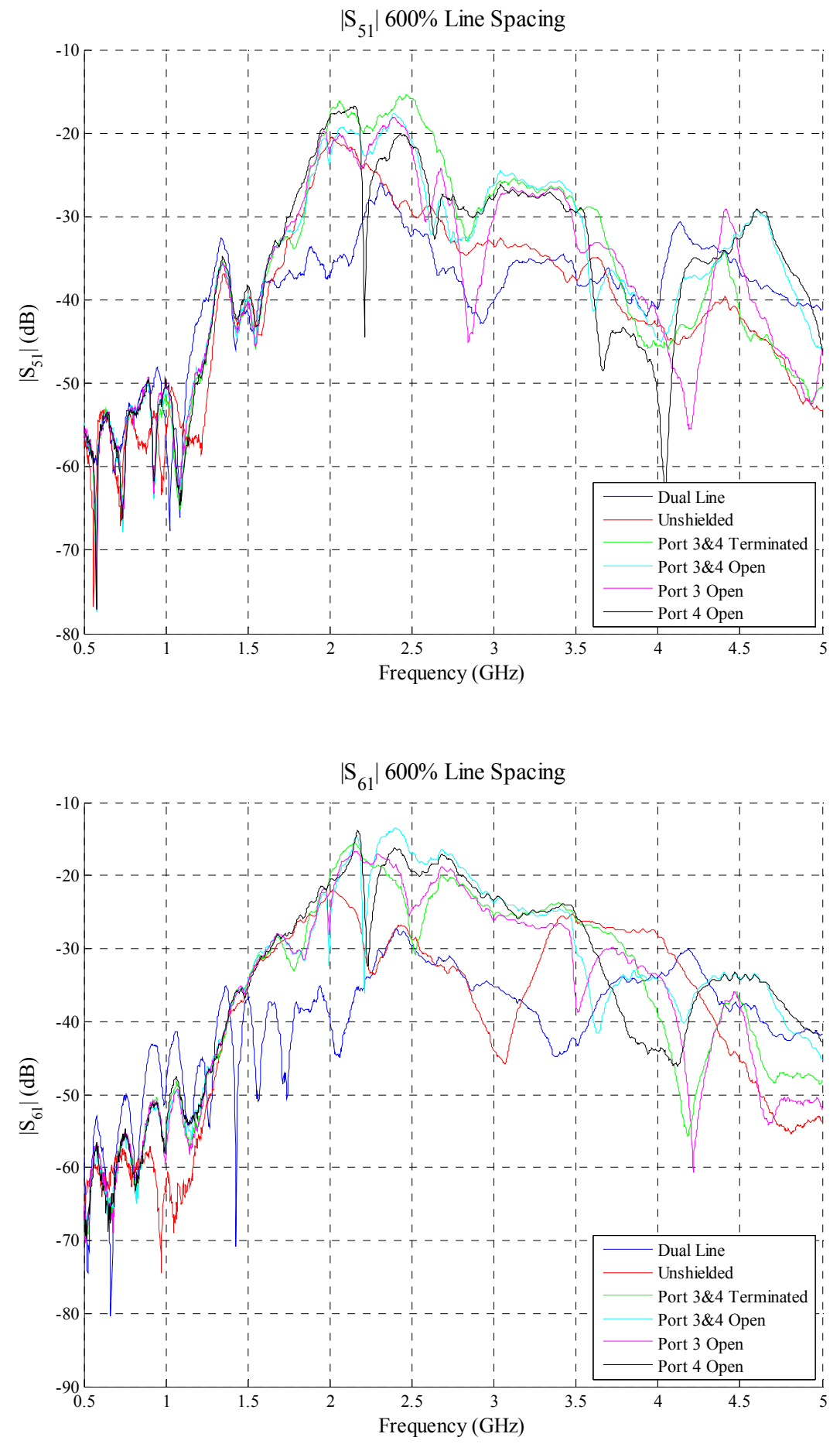

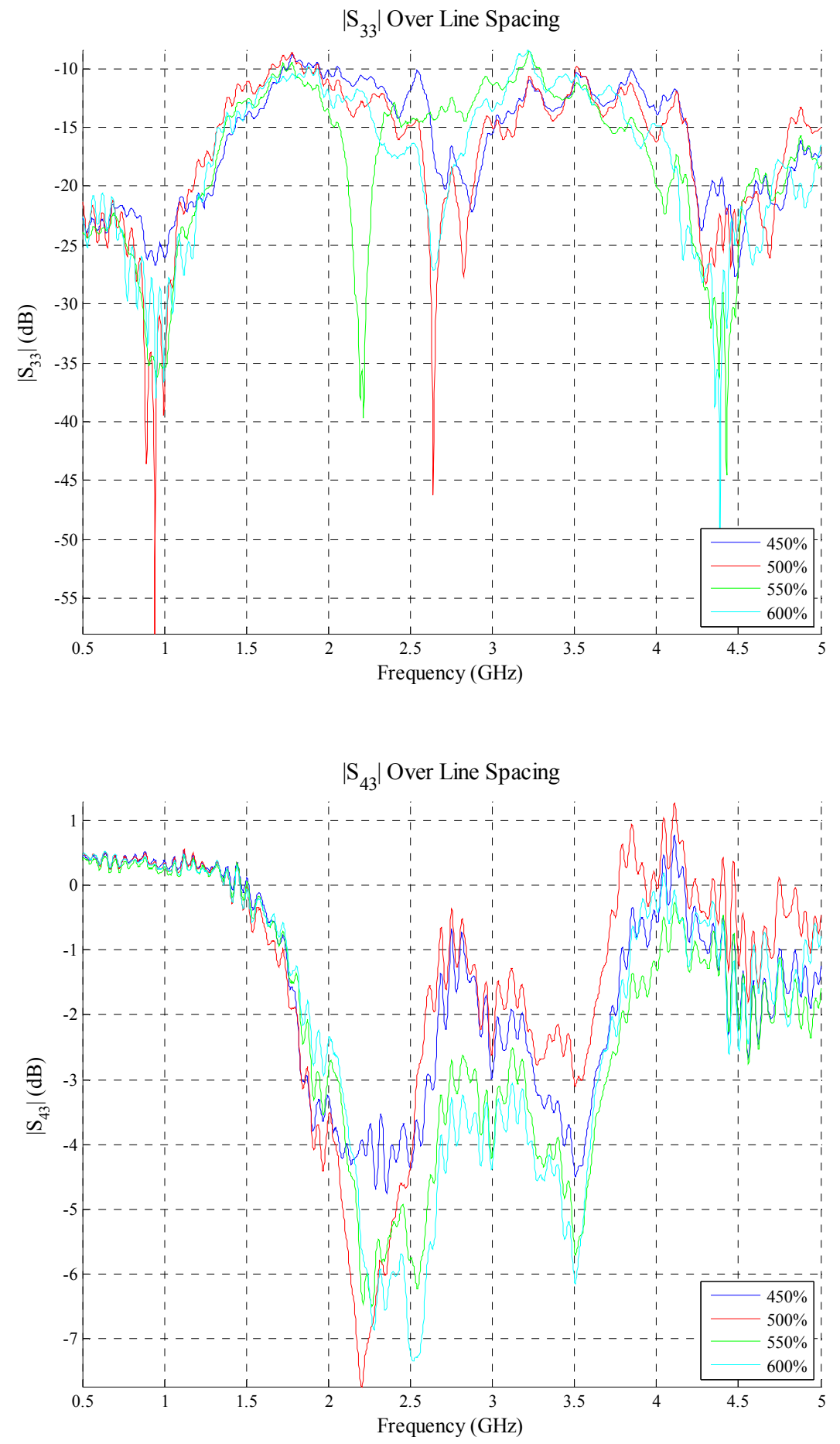

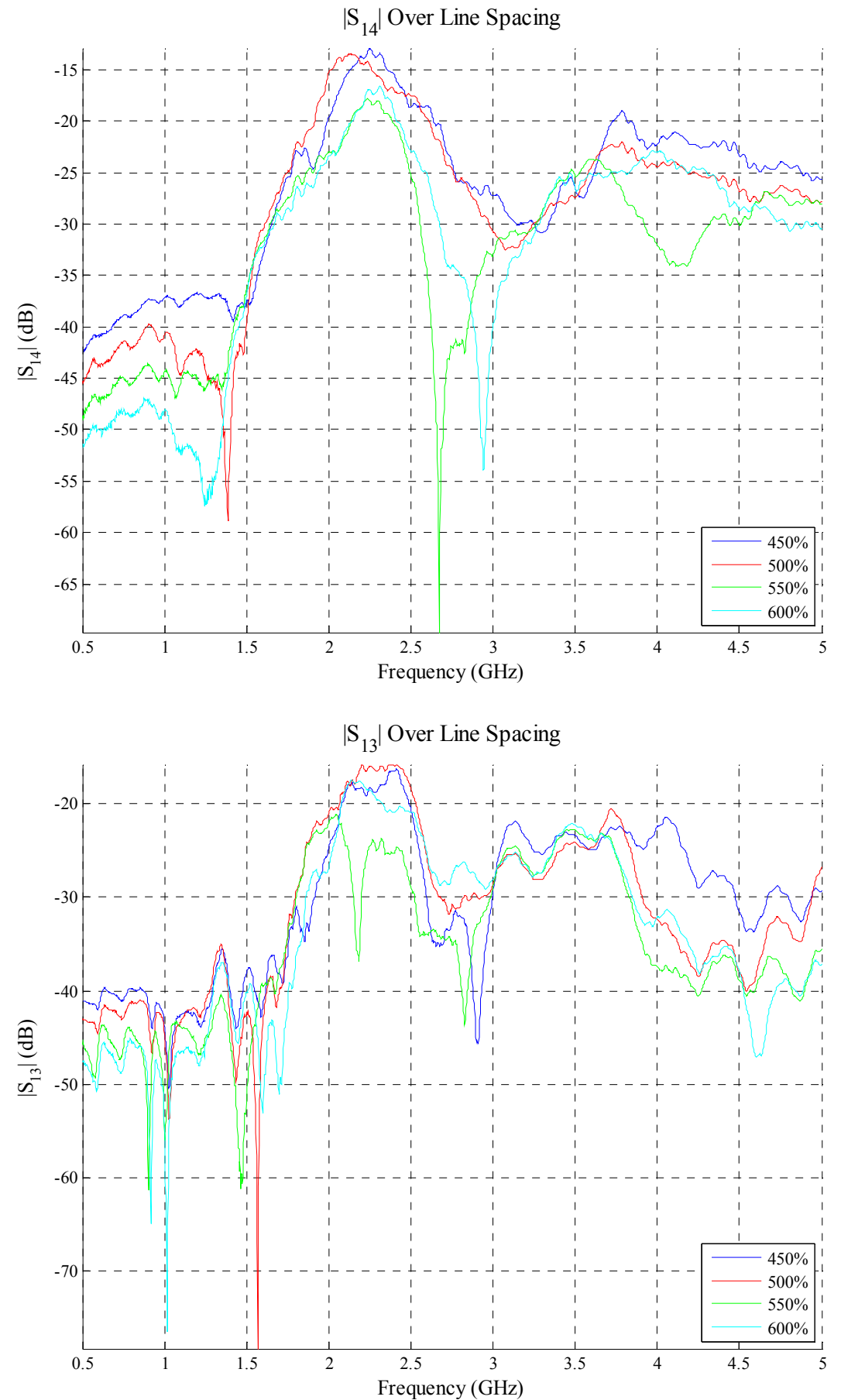